University of South Florida

DIGITAL COMMONS

@ UNIVERSITY OF SOUTH FLORIDA
Digital Commons @ University of

South Florida

$8-1-2010$

\title{
Travel Assistant Device - Deployment to Transit Agencies
}

CUTR

Follow this and additional works at: https://digitalcommons.usf.edu/cutr_nctr

\section{Recommended Citation}

"Travel Assistant Device - Deployment to Transit Agencies," National Center for Transit Research (NCTR) Report No. CUTR-NCTR-RR-2009-03, Center for Urban Transportation Research, University of South Florida, 2010.

DOI: https://doi.org/10.5038/CUTR-NCTR-RR-2009-03

Available at: https://scholarcommons.usf.edu/cutr_nctr/141

This Technical Report is brought to you for free and open access by the National Center for Transit Research (NCTR) Archive (2000-2020) at Digital Commons @ University of South Florida. It has been accepted for inclusion in Research Reports by an authorized administrator of Digital Commons @ University of South Florida. For more information, please contact digitalcommons@usf.edu. 
Prepared by for Transit Research

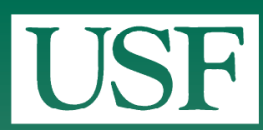

UNIVERSITY OF

SOUTH FLORIDA
National Center

\section{Travel Assistance Device
eployment to Transit Agencies \\ Travel Assistance Device
Deployment to Transit Agencies}

August 2010

Final Report

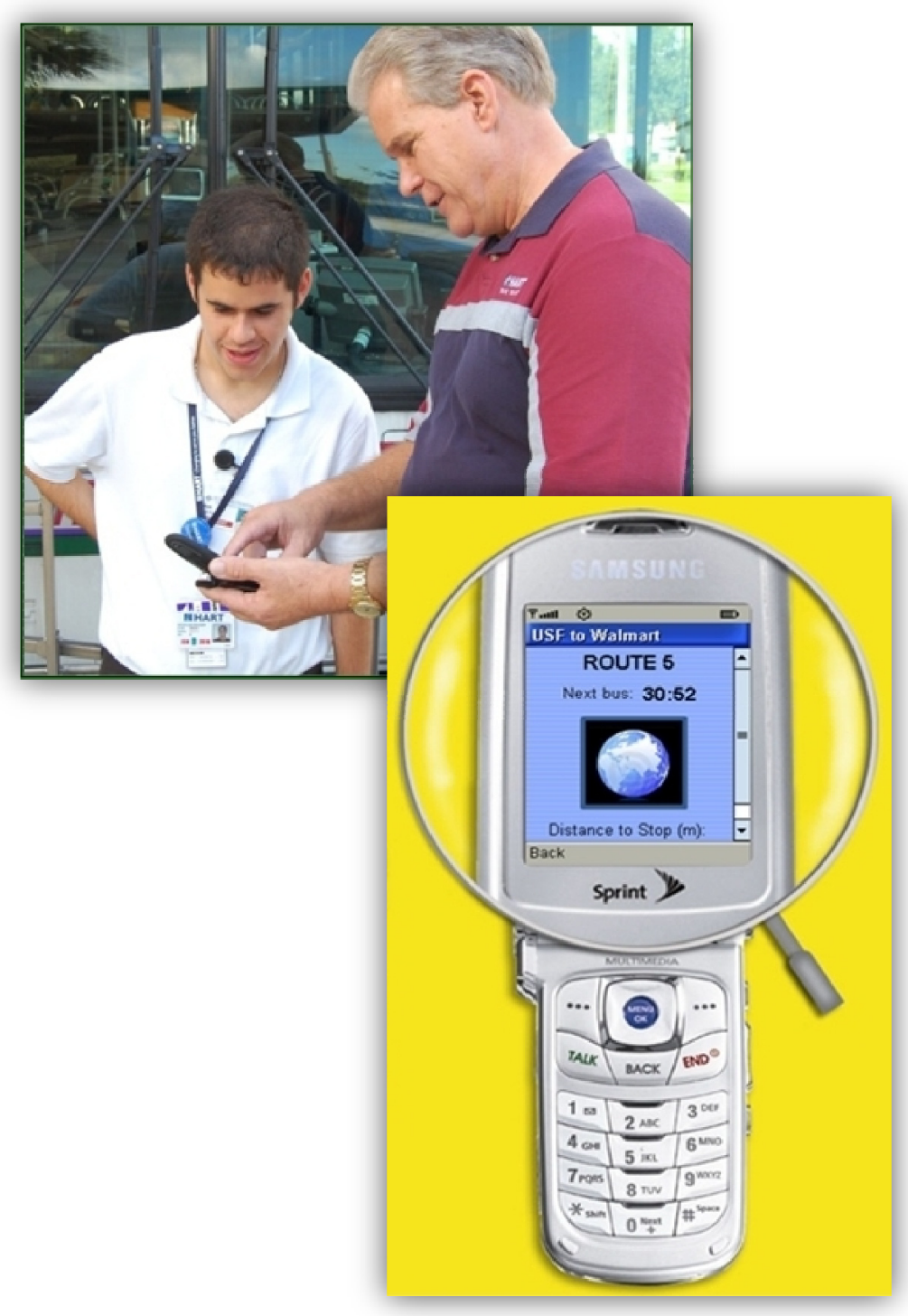

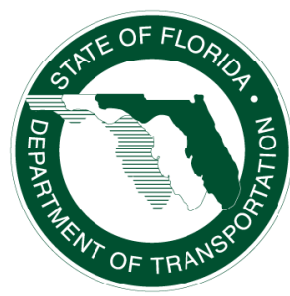

Funded by
Florida Department
of Transportation

Funded by
Florida Department
of Transportation

Funded by
Iorida Department
of Transportation

FDOT BDK85 TWO 977-12 


\title{
Travel Assistance Device Deployment to Transit Agencies
}

\author{
Final Report
}

Prepared for

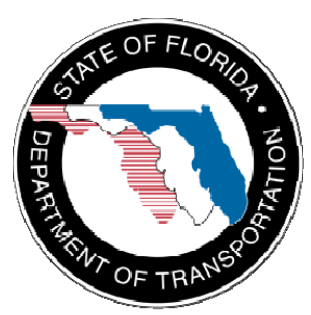

State of Florida Department of Transportation

Public Transit Office

605 Suwannee Street, MS 30

Tallahassee, Florida 32399-0450

Project Manager: Amy Datz

Prepared by

Sean Barbeau, Nevine Labib Georggi, and Philip Winters

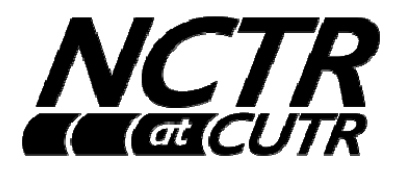

National Center for Transit Research

Center for Urban Transportation Research (CUTR)

University of South Florida

4202 East Fowler Avenue, CUT100

Tampa, Florida 33620-5375

August 2010 
The opinions, findings, and conclusions expressed in this publication are those of the authors and not necessarily those of the State of Florida Department of Transportation. 
Technical Report Documentation Page

\begin{tabular}{|c|c|c|c|c|}
\hline $\begin{array}{l}\text { 1. Report No. } \\
\text { USF } 21177904\end{array}$ & \multicolumn{2}{|c|}{ 2. Government Accession No. } & \multicolumn{2}{|c|}{ 3. Recipient's Catalog No. } \\
\hline \multicolumn{3}{|l|}{ 4. Title and Subtitle } & \multicolumn{2}{|l|}{$\begin{array}{l}\text { 5. Report Date } \\
\text { August } 2010\end{array}$} \\
\hline \multicolumn{3}{|c|}{ Travel Assistance Device (TAD) - Deployment to Transit Agencies } & \multicolumn{2}{|c|}{ 6. Performing Organization Code } \\
\hline \multicolumn{3}{|c|}{$\begin{array}{l}\text { 7. Author(s) } \\
\text { Sean Barbeau, Nevine Labib Georggi, and Phil Winters }\end{array}$} & \multicolumn{2}{|c|}{ 8. Performing Organization Report No. } \\
\hline \multirow{2}{*}{\multicolumn{3}{|c|}{$\begin{array}{l}\text { 9. Performing Organization Name and Address } \\
\text { National Center for Transit Research } \\
\text { Center for Urban Transportation Research } \\
\text { University of South Florida } \\
\text { 4202 East Fowler Avenue, CUT100, } \\
\text { Tampa, FL 33620-5375 }\end{array}$}} & \multicolumn{2}{|c|}{ 10. Work Unit No. (TRAIS) } \\
\hline & & & \multicolumn{2}{|c|}{$\begin{array}{l}\text { 11. Contract or Grant No. } \\
\text { BDK85 TWO } 977-12\end{array}$} \\
\hline \multirow{2}{*}{\multicolumn{3}{|c|}{$\begin{array}{l}\text { 12. Sponsoring Agency Name and Address } \\
\text { Florida Department of Transportation } \\
\text { Public Transit Office } \\
\text { 605 Suwannee Street, MS } 30 \\
\text { Tallahassee, FL 32399-0450 }\end{array}$}} & \multicolumn{2}{|c|}{$\begin{array}{l}\text { 13. Type of Report and Period Covered } \\
\text { FINAL }\end{array}$} \\
\hline & & & \multicolumn{2}{|c|}{ 14. Sponsoring Agency Code } \\
\hline \multicolumn{5}{|c|}{$\begin{array}{l}\text { 15. Supplementary Notes } \\
\text { FDOT Project Manager - Amy Datz, State Transit Environmental Planner }\end{array}$} \\
\hline \multicolumn{5}{|c|}{ 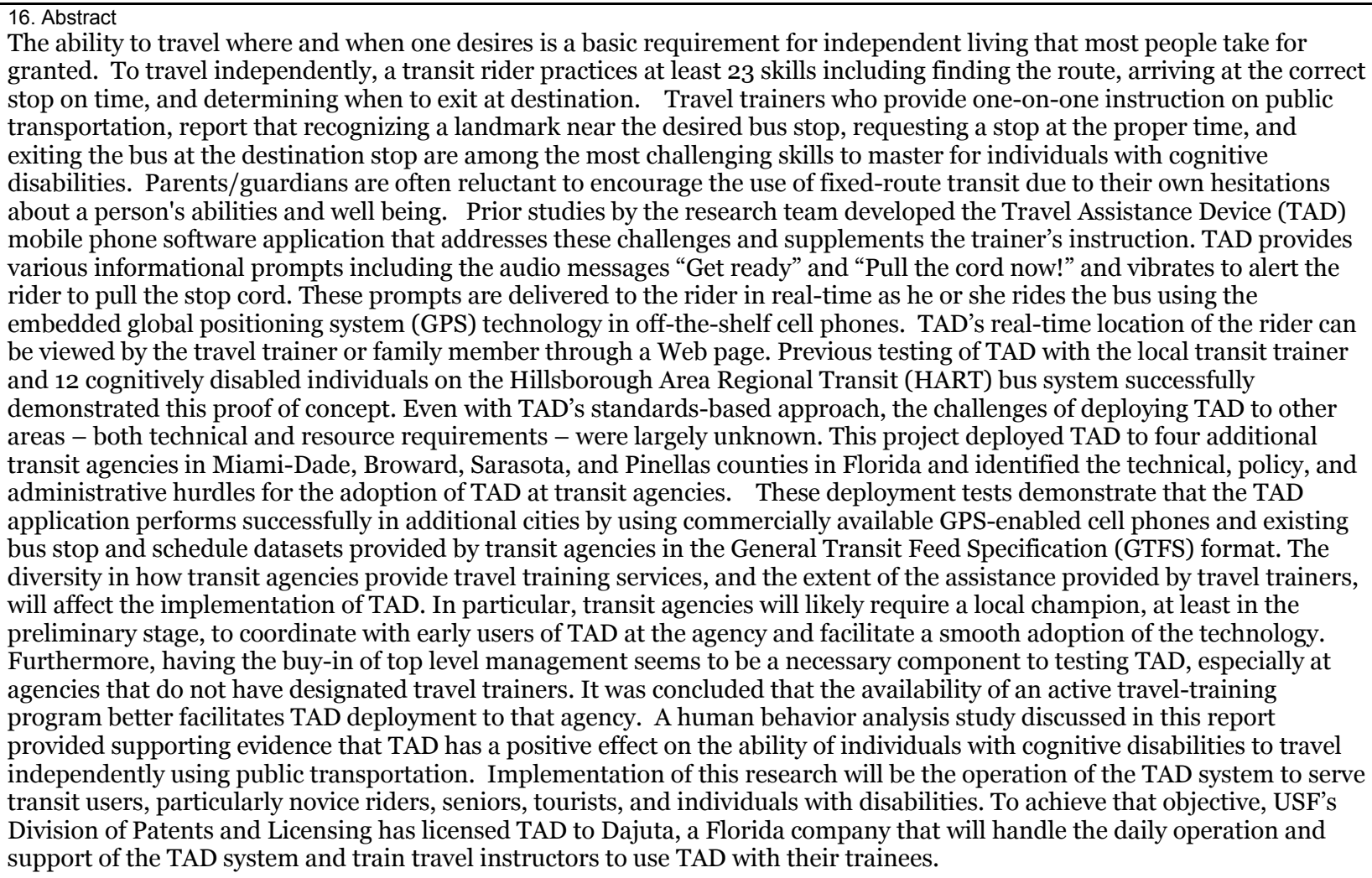 } \\
\hline \multicolumn{2}{|c|}{$\begin{array}{l}\text { 17. Key Word } \\
\text { Global positioning systems, assistive technologies, } \\
\text { travel assistance device, mobile phone, cellular } \\
\text { phone, mobile navigation device, travel training, } \\
\text { mobility training. }\end{array}$} & \multicolumn{3}{|c|}{$\begin{array}{l}\text { 18. Distribution Statement } \\
\text { Available to the public through the National Technical Information } \\
\text { Service (NTIS), 5285 Port Royal Road, Springfield, VA 22161, (703) } \\
\text { 487-4650, http://www.ntis.gov//, and through the NCTR Web site at } \\
\text { http://www.nctr.usf.edu/. }\end{array}$} \\
\hline $\begin{array}{l}\text { 19. Security Classif. (of this report) } \\
\text { unclassified }\end{array}$ & $\begin{array}{l}\text { 20. Security Classi } \\
\text { unclassified }\end{array}$ & f this page) & $\begin{array}{l}\text { 21. No. of Pages } \\
102\end{array}$ & 22. Price \\
\hline
\end{tabular}




\section{Acknowledgements}

This report was prepared by the National Center for Transit Research (NCTR) through the sponsorship of the Florida Department of Transportation (FDOT) and the U.S. Department of Transportation.

University of South Florida (USF) CUTR Project Team

Sean Barbeau, Principal Investigator and Research Associate, CUTR

Nevine Labib Georggi, Co-Principal Investigator and Senior Research Associate, CUTR

Philip L. Winters, Co-Principal Investigator and TDM Program Director, CUTR

Miguel Labrador, PhD, Department of Computer Science \& Engineering, COE

Rafael Perez, PhD, Department of Computer Science \& Engineering, COE

FDOT Project Manager

Amy Datz, State Transit Environmental Planner

USF Computer Science and Engineering Student Team

Marcy Gordon, Graduate Research Assistant

Óscar Lara Yejas, Graduate Research Assistant

Theodore Larkins, Research Experience for Undergraduates (REU) Student

Richard Meana, Research Experience for Undergraduates (REU) Student

The research team also acknowledges Arica J. Bolechala for her contribution to this project by conducting testing and analysis of TAD's impact on transit riders with cognitive impairments as part of her master's thesis in the USF Applied Behavior Analysis Master's Program. Portions of her work are documented in this report with permission.

The research team is grateful to the following transit agencies and their staff for volunteering their time and support for TAD field testing.

Broward County Transit

Wendy Novack, Travel Instructor/SPC II, Paratransit Services

Pinellas Suncoast Transit Authority

Debra Woodward, Director of Information Technology

Phil Cao, Systems Analyst

Bob Lasher, Manager of Community Relations

Gabrielle Donaldson, Marketing \& Sales Coordinator

Bill Hughes, Project Manager for the Real Time Bus Information project 


\author{
Miami Dade Transit \\ Rosie Perez, Senior Chief of Information Technology \\ Boon-Choo Tan, Computer Services Senior Manager \\ Hector Garnica, Computer Services Senior Manager \\ Peter Rubio, Systems Analyst/Programmer 2 \\ Kiranmai Chirumamilla, Transportation Engineer Intern \\ Sarasota County Area Transit \\ Dominick Locascio, Mobility Coordinator \\ Jason Harris, Business Data Analyst \\ Mark Leffingwell, Mobility Trainer \\ Hillsborough Area Regional Transit \\ Mark Sheppard, Travel Trainer
}

The research team thanks the Sprint-Nextel Application Developer Program for its cooperation and the donation of phones and cellular service that were used as part of this research project. 


\section{Executive Summary}

The ability to travel where and when one desires is a basic requirement for independent living that most people take for granted. Public transit riders practice at least 23 skills to travel independently, including arriving at the correct bus stop on time to catch the bus, boarding the correct bus, and determining when to signal the driver to get off the bus at the correct stop. Travel trainers, who provide one-on-one travel instruction for public transportation, report that the skills required to recognize a landmark near the desired bus stop, signal for exiting at the proper time, and exiting the bus at the right bus stop are among the most challenging skills to master for individuals with cognitive disabilities. Also, parents and guardians are often reluctant to encourage the use of fixed-route transit due to their concerns about the transit rider's abilities and well being.

Prior research by the team developed the Travel Assistance Device (TAD), a software application for global positioning system (GPS)-enabled mobile phones that addresses these challenges. TAD provides various informational prompts while individuals are riding the bus such as auditory and visual messages to Get ready and Pull the cord now! and vibrates to alert the rider to pull the stop cord. Also, the real-time location of the rider can be viewed by the travel trainer or family member through a TAD Web page.

TAD uses stop and route data provided by transit agencies in the de facto industry standard General Transit Feed Specification (GTFS) format (previously known as the Google Transit Feed Specification), which is used to support many Web and mobile applications that provide transit information, including Google Maps.

Previous testing of TAD with the local transit trainer and 12 cognitively disabled individuals in the Tampa, Florida area on the Hillsborough Area Regional Transit (HART) bus system successfully demonstrated this proof of concept system. HART's travel trainer was instrumental in field testing TAD and providing valuable feedback to the research team to refine and continue improvement of the TAD mobile software application. However, while TAD was designed to be deployable to cities beyond Tampa, even with TAD's standards-based approach the actual challenges of deploying TAD to other communities - both technical and resource requirements - were largely unknown. If a transit agency has an accurate and up-to-date bus stop inventory and its data are placed into the GTFS format, the TAD system should be able to automatically import that agency's data into the TAD system. Due to differences in data representation by each transit agency, as well as potential differences in cell phone and GPS performance in different cities, TAD must be evaluated for potential expansion by actually importing GTFS data from cities outside the Tampa, Florida area and performing field tests in these areas.

This project deployed TAD to four additional public transportation agencies in Miami-Dade, Broward, Sarasota, and Pinellas counties in Florida and identified the technical, policy, and administrative hurdles for the adoption of TAD. These deployment tests demonstrated that the TAD application functions successfully in additional cities by using commercially available GPS-

vii • 
enabled cell phones and existing bus stop and schedule datasets provided by transit agencies in the General Transit Feed Specification format. When evaluated in the field in Miami-Dade, Pinellas, Sarasota, and Broward counties, TAD functioned successfully in a similar way to the original TAD field tests in Tampa, Florida. Various technical challenges related to the preparation of TAD for deployment to additional cities, including the process of importing new agency GTFS data, are documented in this report.

All of the technical, policy, and administrative hurdles surpassed during this project will enable future deployments of TAD to new transit agencies to proceed with greater ease. This research reinforced the previous observations that TAD is an excellent complement to travel training, as both have similar goals in aiding riders to use public transportation. In the final deployment to customers, travel trainers can easily introduce TAD to new users since the trainer already assists new or existing transit riders who need help riding public transportation. Travel trainers also have reported that TAD may help reduce the time required to train some new transit riders, which would increase trainer productivity and enable them to train more riders. TAD is therefore a tool for both travel trainers and transit riders that can help users get off the bus at the correct place and time.

During this project the research team also discovered that the diversity in how transit agencies provide travel training services, and the extent of the assistance provided by travel trainers, will affect the implementation of TAD. In particular, transit agencies will likely require a local champion, at least in the preliminary stage, to coordinate with early users of TAD at the agency and facilitate a smooth adoption of the technology. The research team originally planned to replicate the successful testing model used in the Tampa Bay area, which partnered with HART's travel trainer to help evaluate TAD in field tests. HART's travel trainer was able to take a TAD cell phone with him as he traveled the transit system as part of his daily duties, and record a significant amount of data about TAD's performance and provide valuable feedback to the research team. However, it was discovered that three out of the four transit agencies do not employ travel trainers as part of their staff. Broward County is the only agency that employs a travel trainer in the same capacity as HART's travel trainer.

In the absence of a travel trainer, the research team was directed to a variety of personnel to assist with the field tests, including paratransit coordinators, marketing specialists, customer service representatives, and technology programmers/analysts. The staff of all partnering agencies were very enthusiastic about the TAD concept and its potential in aiding transit riders, but due to the demands of their normal job duties these personnel were unable to dedicate the same amount of time to field testing TAD as had been committed by HART's travel trainer in the first phase of the TAD project. For final deployment to customers at agencies without travel trainers, a discussion with the transit agency would be necessary to identify the department that most closely aligns itself with helping transit customers to navigate using fixed-route transit. This may fall into the customer service department in some transit agencies. For example, in the absence of travel training, transit ambassador programs that assist some customers who have questions at transit stops may be a good way to introduce TAD to transit riders.

Based on the findings documented in this report, the next step in the evolution of TAD is the integration of TAD within the travel training curriculum. Future work should examine current 
travel training curriculums and education methods and provide guidance to travel trainers on the use of TAD within their training sessions. Since TAD is useful to many different individuals, both with and without different types of disabilities, the specific integration of TAD into the curriculum may differ depending on the trainee's capabilities. Easter Seals Project ACTION and the Association of Travel Instruction should be engaged in this effort due to their expertise in the travel training field. Given the success of the study detailed in Chapter 5, additional behavior analysis research could provide valuable information to inform these efforts. Additional specific areas of recommended future behavior analysis research are discussed in a later section in this chapter.

Implementation of this research will be the operation of the TAD system to serve transit users, particularly novice riders, seniors, tourists, and individuals with disabilities. To achieve that objective, USF's Division of Patents and Licensing has licensed TAD to Dajuta, a Florida company that will handle the daily operation and support of the TAD system and train travel instructors to use TAD with their trainees. This company will resolve various technical support issues as they arise, including distributing application updates, and will maintain a direct relationship with travel trainers using TAD with their clients. It is anticipated that USF will need to facilitate the initial transition of TAD to the third party for its launch as a service and will also continue to be involved in the development of TAD in order to address research questions as they arise.

At the conclusion of the original TAD study, additional testing with individuals with cognitive disabilities under controlled conditions was recommended to provide evidence of increased independence and improved quality of life for transit riders. This report includes information on a 2010 study led by Arica J. Bolechala, a master's student in the USF Applied Behavior Analysis Master's Program, and facilitated by research team members, which provides supporting evidence that TAD is an effective device for prompting individuals to pull the cord to indicate their bus stop and to exit the bus at the appropriate location. Without TAD, study participants did not pull the cord to exit the bus at the appropriate times, which increases the probability that an individual would miss the stop and ultimately get lost. When the participants did carry a $\mathrm{TAD}$, prompts delivered by TAD enabled all of the participants to pull the cord and exit the bus at the correct locations.

There are many areas of potential future work for human behavior analysis related to TAD. For example, an inclusion and exclusion criteria should be developed to help travel trainers determine how trainees are appropriate users of TAD, and additional testing is desired with a more diverse population to determine if TAD can effectively be used by different population groups. 


\section{Table of Contents}

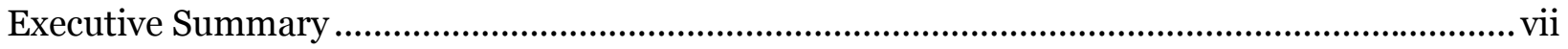

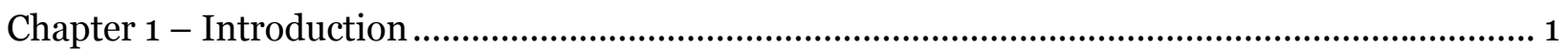

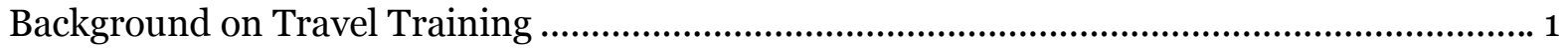

Background on the Travel Assistance Device (TAD) ............................................................. 3

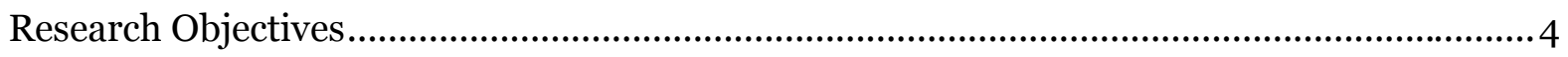

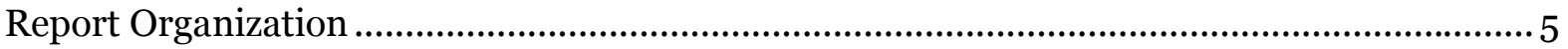

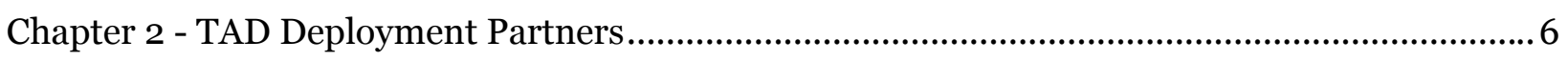

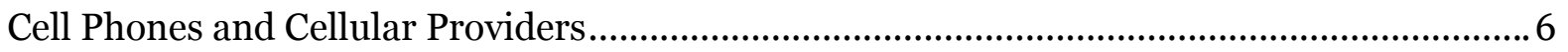

Transit Agencies ................................................................................................................. 10

Selecting TAD Deployment Partners ............................................................................11

Chapter 3 - Pre-Deployment Improvements to the TAD System .............................................. 15

Developing the TAD Informational Web Site..................................................................... 15

Improving the TAD Server Configuration for Reliability and Availability ........................... 18

Adjusting the TAD System to Handle GTFS Data from New Agencies .................................. 21

Creating Off-Route Notifications Based on Transit Route Spatial Information ....................22

Resolving TAD System Issues from Integrating Data from Multiple Agencies ......................24

Integrating a Bus Stop Management Tool with the TAD Web Site .......................................25

Maintaining User Data Consistency after Transit Agency GTFS Data Updates ....................26

Memory Optimization for Tracking Screen in the TAD Trip Planning Web Site.................... 34

Enhancing the Appearance of the TAD Mobile Application ..................................................34

Improving the TAD Database Toolkit User Interface \& Capabilities .................................... 36

Chapter 4 - TAD Deployment Synopsis ................................................................................. 38

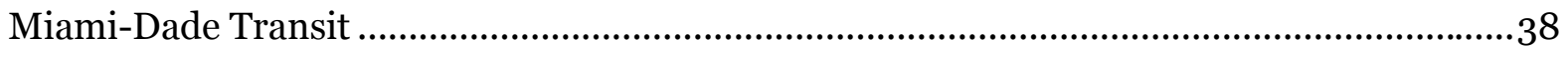

Sarasota County Area Transit ..................................................................................... 41

Pinellas Suncoast Transit Authority ................................................................................46

Broward County Transit ................................................................................................. 53

Discussion of Results of TAD Deployment ...................................................................... 56

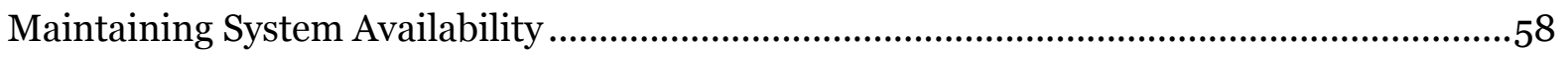

Chapter 5: Evaluating the Effectiveness of TAD on the Bus Riding Behavior of

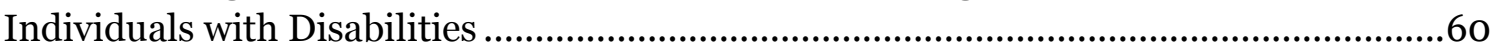

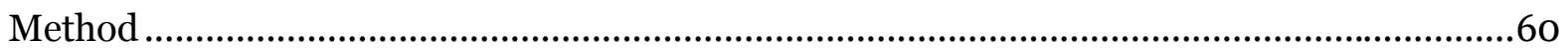

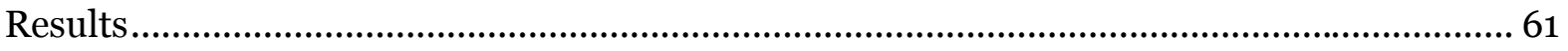

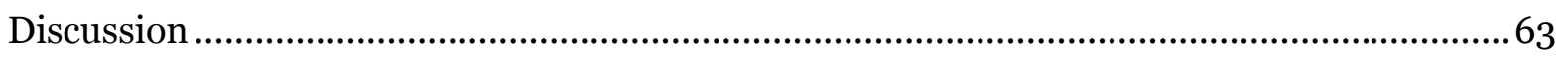




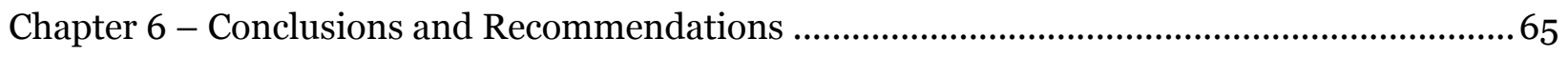

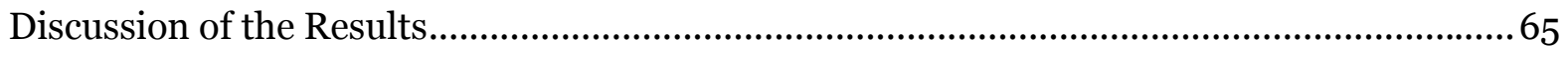

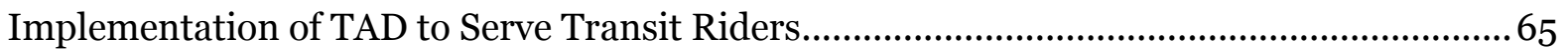

Possible Funding Sources for TAD Implementation.......................................................66

Recommendations for Future TAD Deployments - Partnering with Travel Training

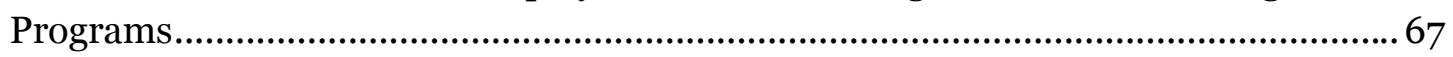

Suggested Future Improvements to the TAD System .............................................................69

Recommendations for Future Human Behavior Analysis Research using TAD ....................... 73

Other Research Needs ................................................................................................. 74

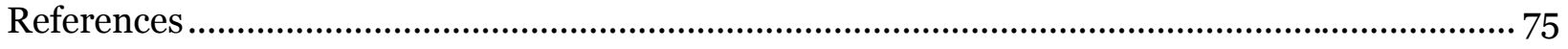

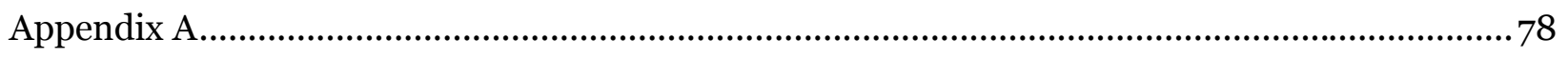

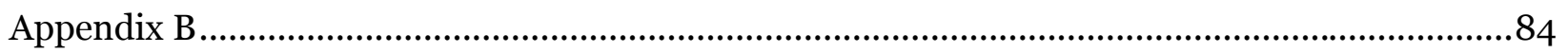

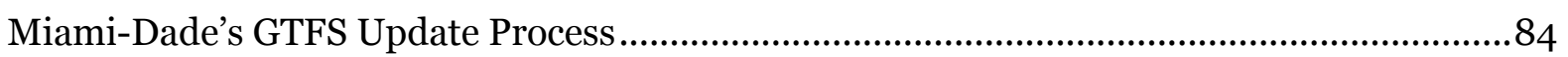




\section{List of Figures}

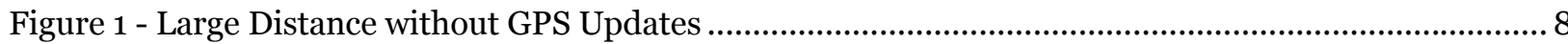

Figure 2 - Comparison of GPS Fixes Reported by the Sanyo Pro 200 and the Sanyo SCP-

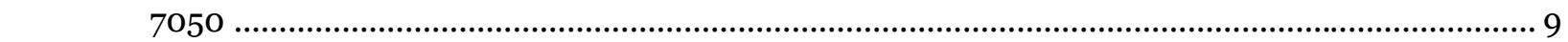

\section{8}

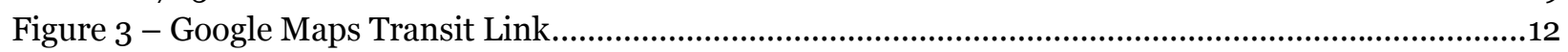

Figure 4 - Basic Online Information on How TAD Works...................................................................16

Figure 5 - Online Step-by-Step Instructions for Web Trip-Planning Tool ....................................................17

Figure 6 - Online Step-by-Step Instructions for Using the TAD Mobile Application .................................18

Figure 7 - Original Server Configuration for TAD ..................................................................................19

Figure 8 - Improved Development/Production Server Configuration ........................................................20

Figure 9 - Straight Line vs. Actual Travel Path between Bus Stops............................................................. 23

Figure 10 - Process for Transforming Shape Data into Geometry Data Used in Off-Route

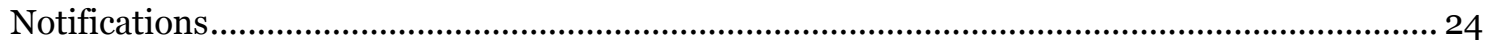

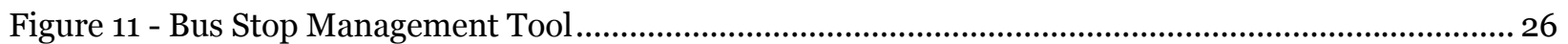

Figure 12 - Data Structure of TAD Trips Database Table .......................................................................... 27

Figure 13 - Required Input for Creating a New Trip on TAD Web Site ..................................................... 27

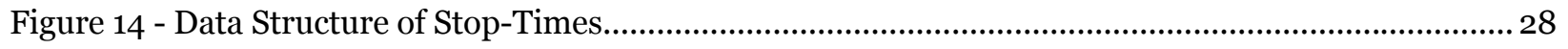

Figure 15 - A Single Intersection on a Route Gives Multiple Sequence IDs ............................................... 29

Figure 16 - Multiple Intersections on a Route Complicate IDs Further ................................................29

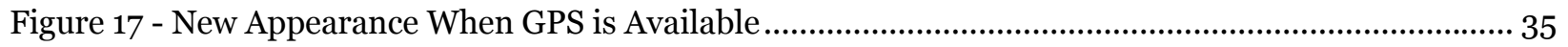

Figure 18 - New Appearance When GPS is Unavailable ........................................................................ 36

Figure 19 - TAD Database Toolkit, Highlighting the Interface Improvements and New

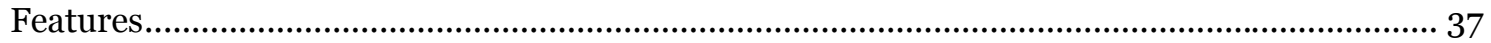

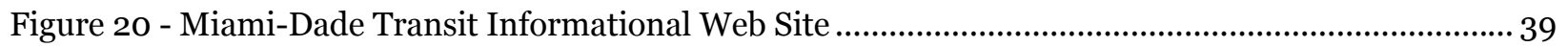

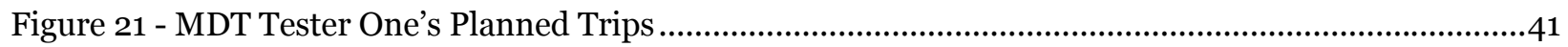

Figure 22 - Sarasota County Area Transit Informational Web Site.......................................................... 42

Figure 23 - Trips Listed by ID when Searching for Trips from SCAT Routes ............................................ 44

Figure 24 - SCAT Tester One's Planned Trips.......................................................................................... 45

Figure 25 - Pinellas Suncoast Transit Authority Informational Web Site.................................................... 47

Figure 26 - Screen Shot of TAD Cell Phone Application with Blank Trip Screen Due to

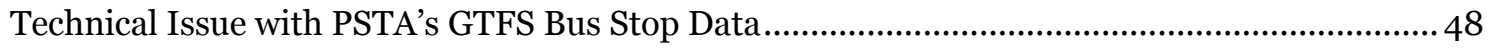

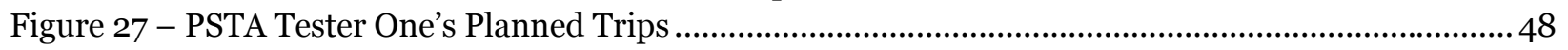

Figure 28 - PSTA Tester One's Trip on 5/26/10 .................................................................................... 50

Figure 29 - End of First Segment of Trip and Beginning of Second Segment where Tracking

is Temporarily Non-Functioning ...............................................................................................51

Figure 30 - TAD Prompts in Relation to the Second to Last (STL) and Ending (End) Bus

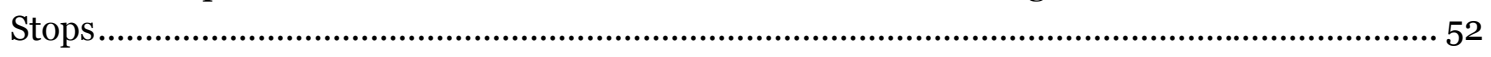

Figure 31 - PSTA Tester Three's Trip from 6/3/10 shows a gap in GPS data............................................. 53

Figure 32 - Broward County Transit Informational Web Site .................................................................... 54

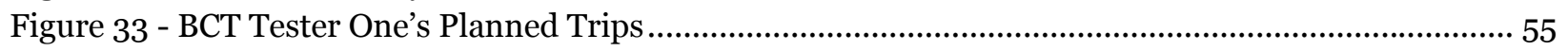

Figure 34 - New Bus Stop Identification Feature on Mouse-Over ............................................................ 58

Figure 35 - New Button to Reset Selected Bus Stops While Creating New Trips......................................... 58

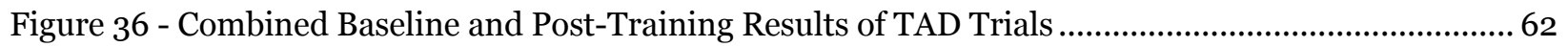

Figure 37 - Suggested Steps to Make TAD Available to Transit Customers .................................................6 66 


\section{List of Tables}

Table 1 - Preliminary Testing Results from using the Sanyo Pro 200 at HART........................................ 10

Table 2 - Field Test Results from PSTA.................................................................................................... 49

Table 3 - Summary of Field Test Results from All Participating Transit Agencies ..................................... 56 


\section{Chapter 1 - Introduction}

\section{Background on Travel Training}

Accessible transportation options increase the ability of people with disabilities to participate in their communities and live independently, in turn affecting their physical, psychological, social, and financial health and well-being. Having additional transportation options leads to more participation by individuals with disabilities in their communities. Many organizations lead the effort in promoting and supporting collaboration of the transportation industry and groups advocating on the behalf of individuals with disabilities to increase mobility in the community.

An agency at the forefront of the effort is the Easter Seals Project ACTION (ESPA). ACTION stands for Accessible Community Transportation In Our Nation [1]. ESPA facilitates training and technical assistance to both members of the disability and transportation communities, helping address diverse accessibility issues. ESPA dedicates resources to the practice of travel training. Travel training (also known as travel instruction) is short-term, one-to-one, intensive instruction designed to teach people, especially seniors and individuals with disabilities, to travel safely and independently on fixed-route public transportation in their community. Seniors and individuals with disabilities learn travel skills while following a specific route, typically to a school or a job site, which is personalized by the travel trainer based on the challenges that each individual might face. The U.S. Department of Transportation has been a longtime partner with ESPA, and on June 30, 2010, Secretary of Transportation Ray LaHood reemphasized the importance of resources such as travel training, especially for youth with disabilities, to help make communities more livable for the 54 million Americans with diagnosis of one or more disabilities [2].

The 2005 report of the National Council on Disability on the state of transportation for people with disabilities acknowledged the strides made in public transportation accessibility since the passage of the Americans with Disabilities Act of 1990 [3]. The report also acknowledged the gaps that remain for many sectors of the disability community, including those who live in rural areas, those who rely on paratransit to get to work or medical appointments, and those with visual impairments who need audible bus stop announcements. The status report also provided recommendations for service improvements and additional research that will lead to greater options for the six million Americans with disabilities who have difficulties obtaining the transportation they need. Among these recommendations, relevant to this research project, is the need for travel training and other efforts to transition paratransit riders to fixed-route. Particularly in the context of the Individuals with Disabilities Education Act (IDEA), the report recommended that transit agencies

1) track and support paratransit riders' successful transition through travel training to fixed-route service and offer transition assistance as needed before denying paratransit eligibility; 
2) ensure that eligibility screening procedures are sufficiently accurate to prevent abuse by individuals who would pay the fare on the fixed-route service;

3) offer a competently staffed travel-training program.

The report also recommended that schools and departments of education ensure that the Individualized Education Program (IEP) process under IDEA includes age-appropriate individualized travel and mobility training.

Although travel training is regarded by the National Council on Disability as an important step toward providing persons with disabilities with mobility, particularly as part of education programs, many transit agencies contacted for the purposes of TAD deployment did not have travel training programs as defined by ESPA and the Association for Travel Instruction (ATI), a professional organization for travel trainers and travel instructors in the United States.

In a 2008 study to assess funding trends and the volume and diversity of services within the travel training instruction field, survey analysis of 74 public transportation providers was conducted [4]. The majority of programs were cited as in-house (57\%), with about one third contracted out to another source (32\%). The remaining 11 percent used a combination of inhouse and contracted services. One full-time staff person provided travel training services in 44 percent of the reporting organizations, with 52 percent of the organizations having more than one staff member.

Travel training increases the mobility and independence of individuals by allowing them to change from paratransit to fixed-route transit service, which also helps the transit agencies by lowering their operating costs. The goal of a free travel training program at Broward County Transit (BCT) is to train eight customers per month. In December 2002, it was reported that an average of 10 persons per month were being travel-trained. The agency estimated an average savings of $\$ 2,300$ for each person who is travel trained, totaling more than $\$ 250,000$ annually, by enabling passengers to use fixed-route, instead of more costly paratransit service [5].

Many agencies struggle with the expensive growth of paratransit. There has been a 5.8 percent increase in paratransit ridership in 2008, according to American Public Transportation Association's (APTA's) 2009 Public Transportation Ridership Report. In 2008, nationwide paratransit ridership made up two percent of public transit ridership but 13 percent of operating costs [6]. Since agencies are prevented by federal law from passing on the additional operating costs of paratransit to the customer, they must find a way to manage this expense [7].

In June 2010, at the 12th International Conference on Mobility and Transportation for Elderly and Disabled People (TRANSED) in Hong Kong, ESPA sponsored Disability Forum: Accessibility for All, where a study was presented that sought to provide travel training stakeholders with a cost benefit analysis model to support decisions about initiating, maintaining, and expanding travel training services [8]. The developed cost-benefit analysis template is suggested for use by transit managers and planners, travel training service providers and advocates from perspectives of trainees, public transportation agencies, and communities. 
ESPA also includes some materials on their Web site to aid in estimating costs and savings of a travel training program.

\section{Background on the Travel Assistance Device (TAD)}

Among the 23 skills an individual has to master to be able to successfully complete a transit trip, knowing when and where to request a stop and actually exit at the correct stop seems to be the most difficult obstacle to overcome to travel independently. Travel Assistance Device (TAD) is a software system that enables global positions system (GPS)-enabled mobile phones to provide real-time navigation prompts to a person using public transportation. National Center for Transit Research (NCTR) researchers developed three services during the initial phase of TAD development [9].

$\square$ Delivery of real-time auditory prompts to the transit rider via a cell phone informing them when they should request a stop

$\square$ Delivery of an alert to the rider, caretaker, and travel trainer when the rider deviated from his/her expected route

$\square$ A Web page that allows travel trainers and caretakers to create new itineraries for transit riders and allows monitoring of real-time rider location

While the research team created TAD to aid transit riders with special needs to increase their level of independence, any new transit rider can benefit from its service. TAD system design had three primary requirements:

$\square$ Make the system low-cost and widely accessible.

This system requirement resulted in the team using off-the-shelf, consumer-grade, GPSenabled cell phones for a compact, inexpensive, all-in-one solution. The system design team used standards-based solutions to allow interoperability on multiple platforms (i.e., different cell phones and cellular carriers). The team also used open-source solutions, when possible, to reduce implementation and management costs to transit agencies. Finally, the team designed the software to be forward-compatible with future mobile devices to minimize future development costs.

- Make the architecture modular so system components can be reused when possible and new features can be added easily in future versions.

The research team used Web services, which allow TAD systems to operate in heterogeneous computing environments on multiple platforms and operating systems. In addition, the team encapsulated the system entities so elements (i.e., database server) may be exchanged without disrupting the entire system.

口 Provide a reliable service simple enough to help transit riders with special needs.

The research team designed a simple, uncluttered user interface. Only two audible and visual announcements need to be made, "Get ready" and "Pull the cord now!" This 
minimizes any possible confusion for the user. The phone also vibrates when the user should request the bus to stop.

A software application was also created that allows additional transit agencies to be added to the TAD system if they format their route and stop data into the General Transit Feed Specification (GTFS), (formerly known as the Google Transit Feed Specification, http://code.google.com/transit/spec/transit feed specification.html), which has become a de facto standard for transit route and scheduling data, with 125 U.S. transit agencies currently participating in Google Transit.

Since 2007, when prototype-testing using the Hillsborough Area Regional Transit (HART) transit system in Tampa began, TAD has been continuously refined. Limited field tests of TAD were conducted in Tampa with a dozen cognitively disabled individuals on HART bus system, which successfully demonstrated this proof-of-concept. Many other transit agencies have expressed interest in using TAD.

This project took the next step and deployed TAD to additional public transportation agencies and identified the technical, policy, and administrative hurdles for the adoption of TAD.

TAD has also received funding under the Transportation Research Board's IDEA Program. This project developed a communications link between TAD and an Automatic Vehicle Location (AVL) system implementation at HART [10]. This communication supports advanced TAD features based on the real-time location of the transit vehicle in relationship to each rider's realtime location. Such features include providing personalized notices via the rider's mobile phone that estimate when the bus will reach his/her current position and notifying him/her when the specific bus arrives.

The TAD/AVL system integration project produced many pieces of useful information, including the design and development of an information distribution model for transit agencies that would make real-time AVL data accessible to outside agencies for a variety of public services without further cost to the agency or transit system. The project documented the system design of the TAD/AVL integration as well as the different design approaches so that agencies better understand the pros and cons of different design decisions. The project also demonstrated innovation in the development of new methods of personalized communication of transit information to individuals through their mobile phones, and also supports the general investment by other public or private agencies in transit information distribution by providing easily accessible real-time AVL information.

\section{Research Objectives}

This research project focused on the deployment of TAD to additional Florida transit agencies. The research evaluated the level of effort required to establish the TAD system in a new community, performed field-testing on TAD at each of the agencies, and analyze the performance of TAD at these agencies. 
Before deploying TAD to additional transit agencies, many enhancements and adjustments were made to improve TAD's performance and usability, including

$\square$ developing the TAD informational Web site;

$\square$ improving the TAD server configuration for reliability and availability;

$\square$ adjusting the TAD system to handle transit information for new agencies;

$\square$ creating off-route notifications based on transit route spatial information;

$\square$ integrating a bus stop management tool to correct bus stop errors;

$\square$ maintaining user data consistency after transit agency GTFS data updates;

$\square$ optimizing memory for tracking screen in TAD trip planning Web page;

$\square$ enhancing the appearance of TAD mobile application;

$\square$ improving TAD database toolkit.

\section{Report Organization}

After this introductory chapter, Chapter 2 details efforts to engage more cell carriers and test TAD on a variety of cell phones, as well as the selection criteria used for transit agency partners. Chapter 3 describes the tasks involved in making TAD enhancements to improve its performance and usability. Chapter 4 provides a synopsis of the actual deployment to the transit agencies. Chapter 5 documents a scientific study in applied behavior analysis. Chapter 6 discusses the findings of this project and recommends future research that will benefit transit riders. 


\section{Chapter 2 - TAD Deployment Partners}

Partners for TAD deployment included cell phone carriers (in addition to Sprint, the only carrier who originally granted permission to test TAD on devices on its network) and transit agencies (other than HART). The next section describes efforts with cellular providers, and the remainder of the report documents the criteria used to select partner transit agencies.

\section{Cell Phones and Cellular Providers}

To facilitate the deployment of TAD to multiple transit agencies and passengers, the research team approached Sprint-Nextel, AT\&T, Alltel, Verizon, and T-Mobile to (1) evaluate support for Java-based standards necessary for the TAD application to run on cell phones on the carrier's network and (2) allow the TAD application to be tested and commercially deployed on their devices and networks.

The research team contacted AT\&T, Verizon, and T-Mobile to request permission to test Java ME applications on mobile devices on their network. Since Verizon and AT\&T serve the majority of U.S. cellular customers (ranked number one and number two carriers in number of total subscribers, respectively), the research team focused on gaining access and testing on these two networks. Verizon purchased Alltel in early 2009 (http://news.VZw.com/news/2009/01/pr2009-01-09.html), and therefore Verizon is now the primary carrier for all customers previously on Alltel. T-Mobile appears to have closed its developer program (http://moconews.net/article/419-t-mobile-usa-shutters-developerprogram-amidst-app-boom/) as of May 2010, and therefore the current status of signing applications for devices on T-Mobile is unknown. TAD already has been successfully tested on devices on the Sprint and Nextel networks.

\section{AT\&T}

The research team successfully completed the AT\&T registration process to become an official application developer for Java Micro Edition (Java ME) devices on the AT\&T network and gained access to the signing tools necessary to allow a location-aware application to run on an AT\&T device.

The research team performed installations of test Java ME applications that use the same functionality as the TAD application (e.g., GPS, wireless communication, data storage on phone) on the AT\&T Samsung Bound phone in order to determine if the phone properly supports the standards used in TAD software development. Currently, despite specifications that indicate that the phone supports Java ME standards, the phone failed to execute several of the tests properly. Troubleshooting these problems is a difficult process since no debugging tools exist for the Samsung Bound and, therefore, tracking down the exact source of the error in the phone platform is very complex. The team narrowed down the point of failure in the Java ME platform to wireless communication using the User Datagram Protocol (UDP) used by TAD to transfer location data back to the server. The team concluded that the Samsung Bound phone was not 
comparable to Sprint devices used for TAD in terms of phone software platform maturity and stability. As a result, the Samsung Bound currently is not a suggested device for TAD deployment. It is expected that as additional devices on the AT\&T network enter the market, a more mature and stable Java ME platform will properly support the Java ME standards used in TAD. Therefore, while the Samsung Bound is not recommended for use with TAD, future AT\&T devices likely will be able to run TAD pending necessary testing of TAD on each device.

\section{Verizon and T-Mobile}

The team identified a contact at Verizon but, despite many requests, was unable to secure access to test TAD on devices on its network. It currently appears that the most promising method of making TAD available on devices on the Verizon network is to create a version of TAD on the Android platform, an open Java-based platform for smart phones that is not specific to one device manufacturer or cellular network. Since the Android platform has fewer variations in implementation and compatibility among different devices than the Java Micro Edition platform, the effort required to create an Android mobile phone application version of TAD may be significantly less than the effort required to port (i.e., transfer) TAD to the many variations of the implementation of the Java ME platform. In other words, more potential customers could be reached with an Android application than a ported version of the Java ME application at a reduced cost of system deployment and maintenance. Additionally, carrier permission is not required to run Android applications on cell phones, and, therefore, the application can be freely developed and deployed based on the schedule of the development team.

Android devices are expected to be available on all major U.S. cellular carriers. Android phones have been launched on the Sprint, Verizon, and T-Mobile networks. AT\&T and Nextel are expected to release Android devices in the second half of 2010. Android passed the iPhone in smart phone market share in the United States in the first quarter of 2010, making it an attractive device for developers who want to reach a large number of users (http://www.npd.com/press/releases/press 100510.html).

\section{Additional testing on Sprint-Nextel}

TAD was also tested on additional Java ME devices (i.e., Sanyo Pro 200, Sanyo Pro 700, and Motorola V950) on the Sprint-Nextel network under this project. The Motorola V950s Java ME platform claimed to meet the standards required by the TAD mobile phone application, but the platform crashed when test applications were run on the phone. Therefore, the Motorola V950 is not recommended as a device for TAD deployment. The Sanyo Pro 200 and 700, which also conform to the standards required by TAD, appear to operate TAD without problems when the phone is flipped open. However, there appears to be an issue causing the phone to occasionally reset once the phone is flipped closed and the application is running in the background. Since the Sanyo Pro 200 and Pro 700 are the phones with the closest features and specification to the Sanyo 7050s originally tested under the TAD Phase One project, additional effort was placed on testing the TAD mobile application with these devices in the field to determine how the GPS within the devices performed. 
The Sanyo Pro 200 is identical in hardware and software to the Sanyo Pro 700. The only difference appears to be in the phone's casing or appearance, with the Pro 700 having a ruggedized and more rubbery case. For the sake of brevity, we will refer to both the Sanyo Pro 200 and 700 as simply the Pro 200 phone.

The research team tested the Pro 200 for several months and began an intense investigation to determine if the Sanyo Pro 200 could be used as the primary deployment device to transit agencies. In initial field tests, the phones constantly lost a GPS signal causing the TAD warning tone to sound often. In examining the trips took with the phones in Google Earth, it became apparent that the phone lost a GPS fix for large gaps of time. Figure 1 shows a distance of 704 meters between GPS fixes reported to the TAD database.

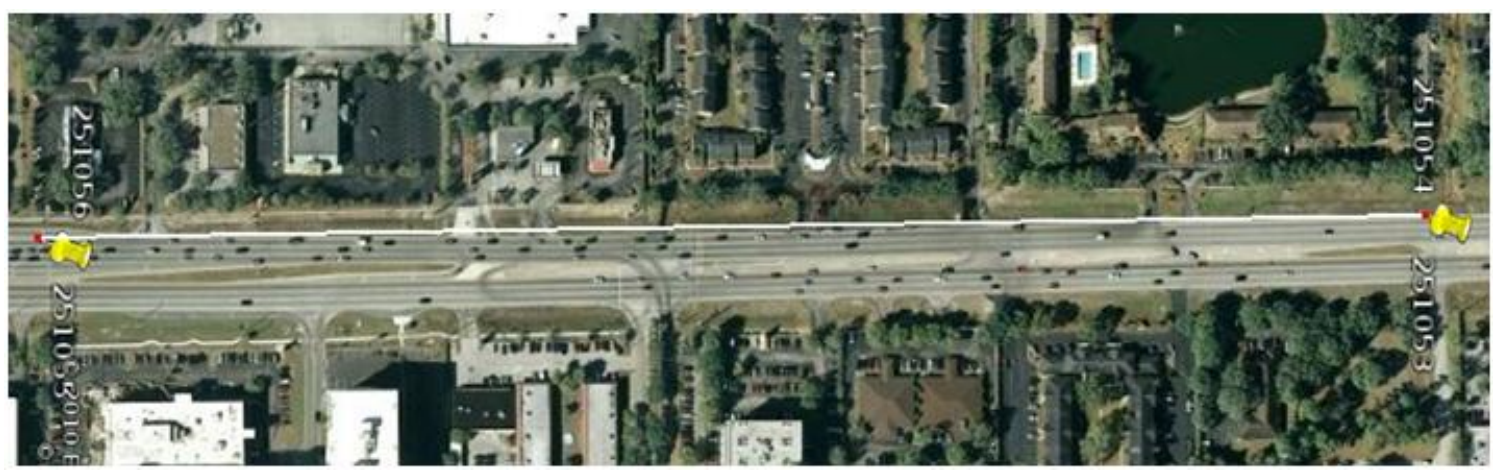

Figure 1 - Large Distance without GPS Updates

Upon further research, it became apparent that the phone was not returning updated GPS coordinates to the TAD mobile application as often as the Sanyo 7050. Often seven or eight GPS in a row would be duplicated on the Pro 200 phones. In Figure 2, we can see that while using a Pro 200 at the exact same time and place as the 7050, the GPS fixes are updated less often on the Pro 200 than on the 7050 when using the same application settings. 

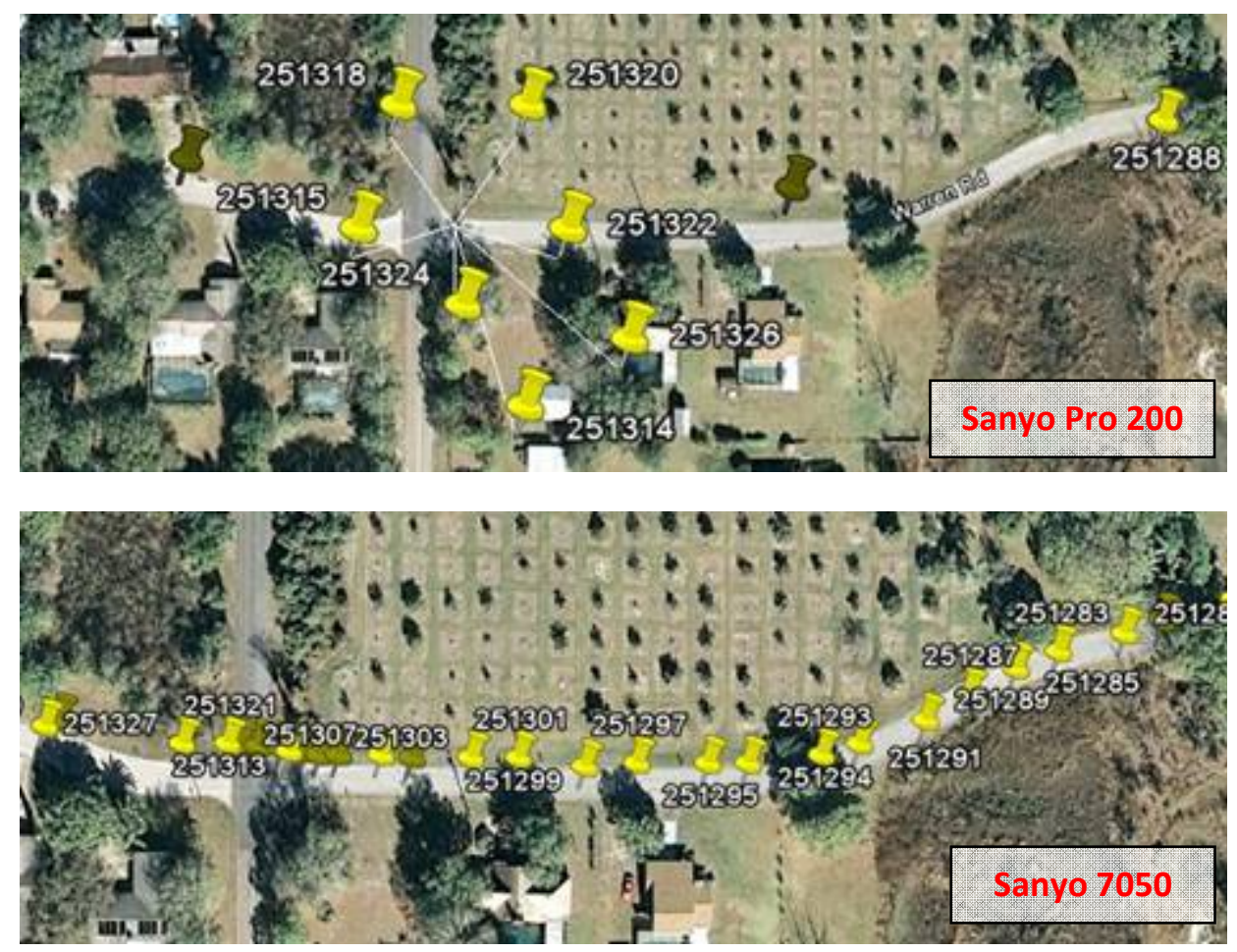

Figure 2 - Comparison of GPS Fixes Reported by the Sanyo Pro 200 and the Sanyo SCP-7050

Just as the team was gaining ground on getting TAD to work smoothly on the Pro 200, all of the Pro 200 phones were accidentally disconnected from Sprint's network and testing had to be put on hold. Later a Sprint technician contacted the research team and explained that there was an issue with the account the phones were provisioned on and the phones had not been activated properly. After the Pro 200 phones were restored to working order, the team requested the assistance of HART Travel Trainer Mark Sheppard to provide his expertise and give feedback on the performance of the Pro 200 phones as he carried the phone during his normal job duties.

Mr. Sheppard planned three trips, with three, four, and three segments respectively. He took these trips on June $9^{\text {th }}$ and then again on June $11^{\text {th }}$. The results, presented in Table 1 , show that while many times the Pro 200 was able to give the Pull the Cord Now alert at the appropriate time, it neglected to give the alert almost as often. The trips planned involved stops that were very close together (often less than 200 meters apart) which is the reason many late prompts were recorded. The prompts were recorded as late if the professional opinion of the HART travel trainer is that the alert was given too late for an average trainee to safely react and pull the cord to get off at the correct stop (given the speed and location of the bus). 
Table 1 - Preliminary Testing Results from using the Sanyo Pro 200 at HART

\begin{tabular}{|l|c|c|c|c|c|}
\hline & $\begin{array}{c}\text { Ideal } \\
\text { Prompts }\end{array}$ & $\begin{array}{c}\text { Early } \\
\text { Prompts }\end{array}$ & $\begin{array}{c}\text { Late } \\
\text { Prompts }\end{array}$ & $\begin{array}{c}\text { No Prompt } \\
\text { Given }\end{array}$ & $\begin{array}{c}\text { Total Number } \\
\text { of Trip } \\
\text { Segments }\end{array}$ \\
\hline Trip 1 & 2 & & 2 & 2 & 6 \\
\hline Trip 2 & 3 & & 4 & 1 & 8 \\
\hline Trip 3 & 2 & 1 & & 3 & 6 \\
\hline Total & 7 & $\mathbf{1}$ & $\mathbf{6}$ & $\mathbf{6}$ & $\mathbf{2 0}$ \\
\hline
\end{tabular}

Due to these results, further tests with the Sanyo Pro 200, and possible adjustments to the TAD mobile application, are required before this device can be used reliably with the TAD mobile application. Future work should investigate if reducing the amount of data being sent to the server in real-time to support the real-time tracking feature of TAD could resolve the GPS frequency update issue. For testing purposes, every fix is sent to the server immediately every second to provide the research team with a complete data set for use in troubleshooting in case an error occurs. However, for real operations after TAD has been thoroughly tested in an area, updated locations could be sent to the server at a reduced update rate while still supporting the Web site tracking feature. If the processing load on the Central Processing Unit (CPU) is impacting the performance of GPS frequency, reducing the communication load may improve the GPS update rate. Future work should also investigate whether the distance thresholds used to trigger the Get Ready and Pull the Cord Now! notifications need to be adjusted to account for this reduced refresh rate, or whether different application settings might be able to produce a more frequent GPS update rate.

Due to these differences in operations between the Sanyo Pro 200 and the Sanyo 7050, the research team decided to use the Sanyo 7050s for the field tests conducted at additional agencies since these devices were the most stable and heavily tested devices with the TAD mobile application. Using the Sanyo 7050s would reduce the chances of unknown errors interrupting testing at the agency and would provide the research team with similar GPS data that could be compared to the original TAD testing conducted in Tampa.

\section{Transit Agencies}

Recognizing the value of having assistance to test on each system, the research team drafted a memorandum of understanding (MOU) that specified what was expected from a participating transit agency and also specified the support afforded by the Center for Urban Transportation Research (CUTR) to the participating agency during the partnership. After distribution to a few transit agencies, it became apparent that agencies treat MOUs as binding contracts, and because signatures were required, the legal counsel of each agency had to be involved. Since a letter format was not subject to these same restrictions, the research team drafted a solicitation letter that was better suited and accepted by the contacted transit agencies. A Frequently Asked Questions (FAQ) list was attached to the letter to address many of the questions that the research team has encountered during initial presentations and discussions with different agencies (Appendix A). 
During transportation or travel-training national conferences, when research team members discussed TAD, they would solicit interested agencies to sign up to partner for TAD deployment. The sign-up list was expanded in response to a February 2009 article on TAD published in Metro Magazine [11].

\section{Selecting TAD Deployment Partners}

The two major criteria for being a TAD deployment partner were:

a) The agency must have its transit data available in GTFS format and be willing to share the URL of that data with the research team to facilitate data import and updates.

b) The agency will designate a travel trainer to work with the team to perform field testing of TAD at the transit agency.

\section{General Transit Feed Specification (GTFS)}

TAD is able to import data from transit agencies if the agency places their data in the GTFS format. The primary criterion for transit agencies to participate in TAD deployment was to have data in the GTFS format so they could plan trips and field test the TAD mobile application. In Florida, the following agencies are included in the Google Transit Partner Program of Google Maps (Figure 3).

- Hillsborough County Regional Area Transit (HART)

- Miami-Dade County Miami-Dade Transit (MDT)

- Pinellas Suncoast Transit Authority (PSTA)

- Broward County Transit (BCT)

- Gainesville Regional Transit System (RTS)

- South Florida Regional Transportation Authority (RTA)

These transit agencies have converted their route, schedule, and bus stop information into the GTFS. According to the Google Transit Partner Program Web site instructions [12], to become a Google transit partner program, a transit agency has to validate and inspect its GTFS using a Feed Validator software tool, zip the files into a file called google_transit.zip, and host the feed on a Web server for Google to retrieve [13]. The agency then contacts Google to complete an online agreement before actual launch. After testing the data in the private preview with Google with satisfactory results, the data are launched and made available inside the Google Maps application so users can plan trips using public transportation provided by that transit agency.

All these agencies and others were pursued to partner with CUTR. Tri-Rail was considered part of MDT for the purpose of TAD deployment. Gainesville RTS was pursued as a candidate for deployment; the request was forwarded to the Center for Independent Living, the agency contracted by RTS to conduct travel training, but they never responded. Further communication efforts with RTS to allow CUTR to download its GTFS to conduct testing were unsuccessful. Though not published on Google's Web site, the Sarasota County Area Transit (SCAT) partnered with CUTR to deploy TAD. CUTR engaged the West Florida Regional Planning Council (WFRPC), which serves counties in Northwest Florida including Bay, Escambia, Holmes, 
Okaloosa, Santa Rosa, Walton, and Washington and their municipalities. WFRPC contracts with the Chautauqua Learn and Serve Charter School for travel training, using New Freedom funds. WFRPC was in the process of making its data available in GFTS and therefore could not test TAD. To their credit, WFRPC staff members were very cooperative and responsive and are still interested in and willing to participate when the data are ready. LYNX, the Central Florida Regional Transportation Authority, also expressed a strong interest in participating in TAD field testing and invited the research team to present the concept to its staff. LYNX outsources its travel training to Quest, Inc.; however, it did not have its transit data in the GTFS format.

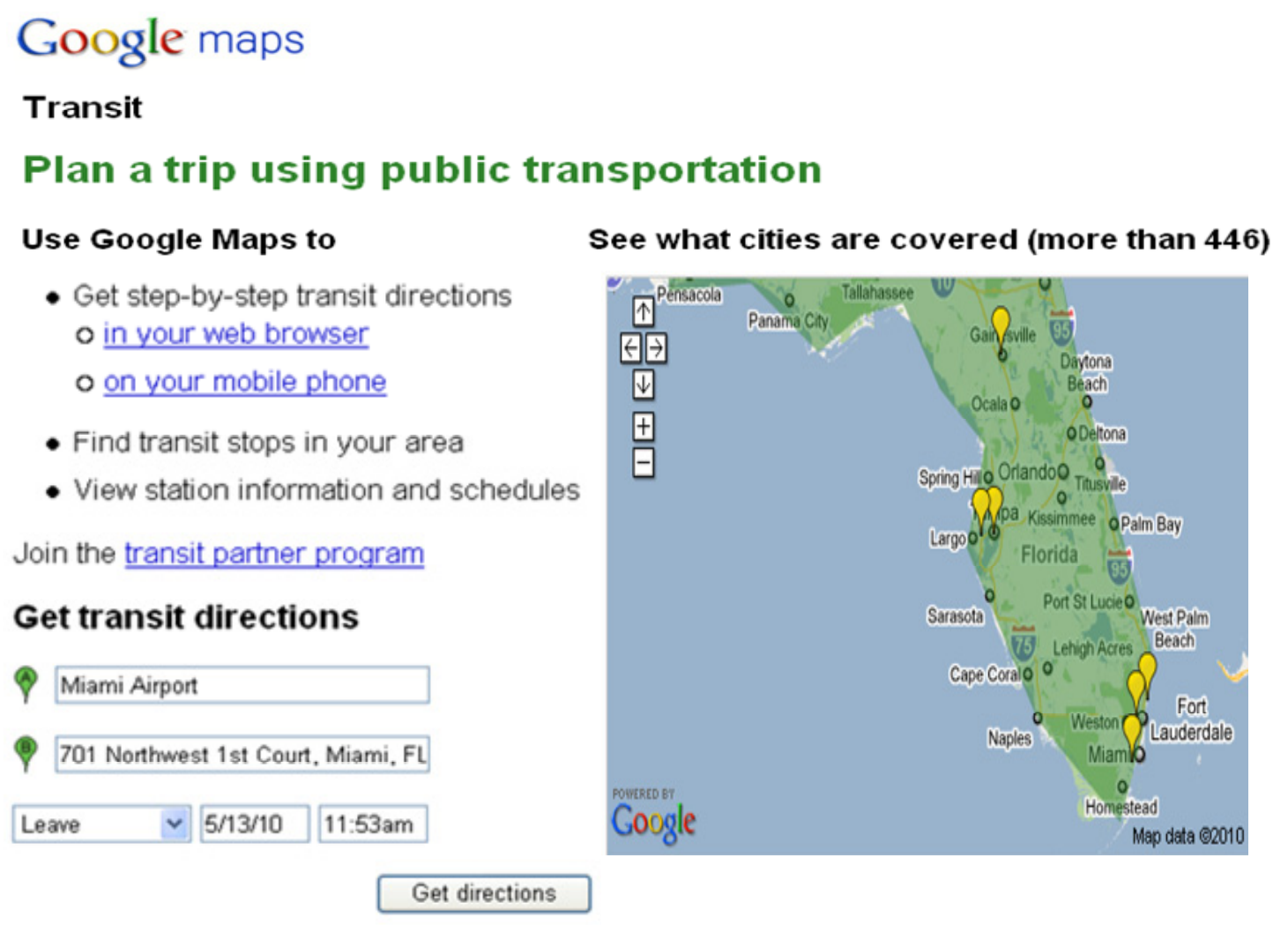

Figure 3-Google Maps Transit Link

TAD follows the same process as Google of retrieving the GTFS data from the transit agency's Web site, both to add new transit agencies to the TAD system and to update the data for an existing transit agency. 
As of May 14, 2010, the following notice was posted on the Google Transit Web site:

Due to overwhelming interest in the Transit Partner Program, we are currently experiencing a significant volume of partner requests. Although we are unable to accept new partners at this time, we encourage you to sign-up in order to be placed on the waiting list.

It is anticipated that more transit agencies will join the Google Transit Maps and trip planning service.

\section{Availability of Travel Trainers}

The second criterion for deploying the TAD prototype to transit agencies was the availability of a travel trainer either directly employed within the agency or subcontracted to perform training activities. Ideally, an agency's travel trainer would assist the project team with performing field tests of $\mathrm{TAD}$, including recruiting individual participants, securing informed consent documents, conducting participant orientation sessions, and helping to evaluate TAD performance on that transit agency's system.

\section{Hillsborough Area Regional Transit}

Among all Florida transit agencies on Google transit, only HART and BCT each have one dedicated travel trainer, as defined by ESPA and ATI. HART has participated in TAD testing since the inception of the prototype and has further assisted in testing as part of the Innovations Deserving Exploratory Analysis (IDEA) 52 - Travel Assistance Device (TAD) to Help Transit Riders [10] . The object of IDEA 52 was to enhance the TAD by providing a link to HART's realtime AVL data. These data can be used to provide services including (1) delivering information to riders via their mobile phone while they are waiting at the bus stop and while they are riding the bus, (2) notifying riders when their specific bus has arrived, (3) providing riders with identifying information so that they board the correct bus if multiple buses are present, and (4) alerting riders and officials if the rider boards the incorrect bus.

HART's travel trainer has performed continuous field testing for the project and provided valuable feedback from the perspective of a travel training professional. He also was instrumental in recruiting six cognitively disabled individuals who previously were traveltrained as part of the initial TAD Phase One project. The trainer contacted the participants and their parents/guardians, introduced them to TAD, answered questions, and obtained the initial consent to sign them up for field testing. He planned a round trip for each individual using the TAD Web site, with each trip customized for each individual to an unfamiliar destination. He was present during the actual field testing exercise and recorded observations during these tests. Since TAD was tested on HART on Phase One and the TAD IDEA projects, HART was not a participant in this TAD deployment project. CUTR originally planned to replicate this successful 
partnership model with the travel training programs at the new TAD deployment sites as part of this project.

Broward County Transit (BCT)

BCT employs a full-time travel trainer who helps individuals learn to use transit in Broward County. The travel trainer at BCT facilitated the partnership between CUTR and the agency to evaluate TAD. The results from testing at Broward County are discussed in Chapter Four. 


\section{Chapter 3 - Pre-Deployment Improvements to the TAD System}

Before deploying TAD to additional transit agencies, many enhancements and adjustments were made to improve TAD's performance and usability. This chapter describes the work that took place to prepare TAD for further field testing by additional transit agencies.

\section{Developing the TAD Informational Web Site}

To facilitate TAD deployment, an informational Web site was developed to provide a centralized location to host information about TAD for interested transit agencies, transit riders, and others. The informational TAD Web site is hosted as a section of the University of South Florida (USF) Location-Aware Information Systems Laboratory Web site and is publicly available at http://www.locationaware.usf.edu/ongoing-research/travel-assistance-device/.

This site includes a description of how the TAD system works (see Figure 4) and information for transit agencies interested in assisting with TAD testing. Step-by-step instructions for various features of the TAD mobile application and Web application were posted for the benefit of TAD testers and were updated as clarifications were needed. Figure 5 shows the Using the TAD Mobile Application Web page, which includes tabs with an overview of the mobile application as well as information on starting and updating TAD on the phone. Figure 6 shows the Using the TAD Web Application Web page, which provides information on planning trips for use in TAD via the Web site. 


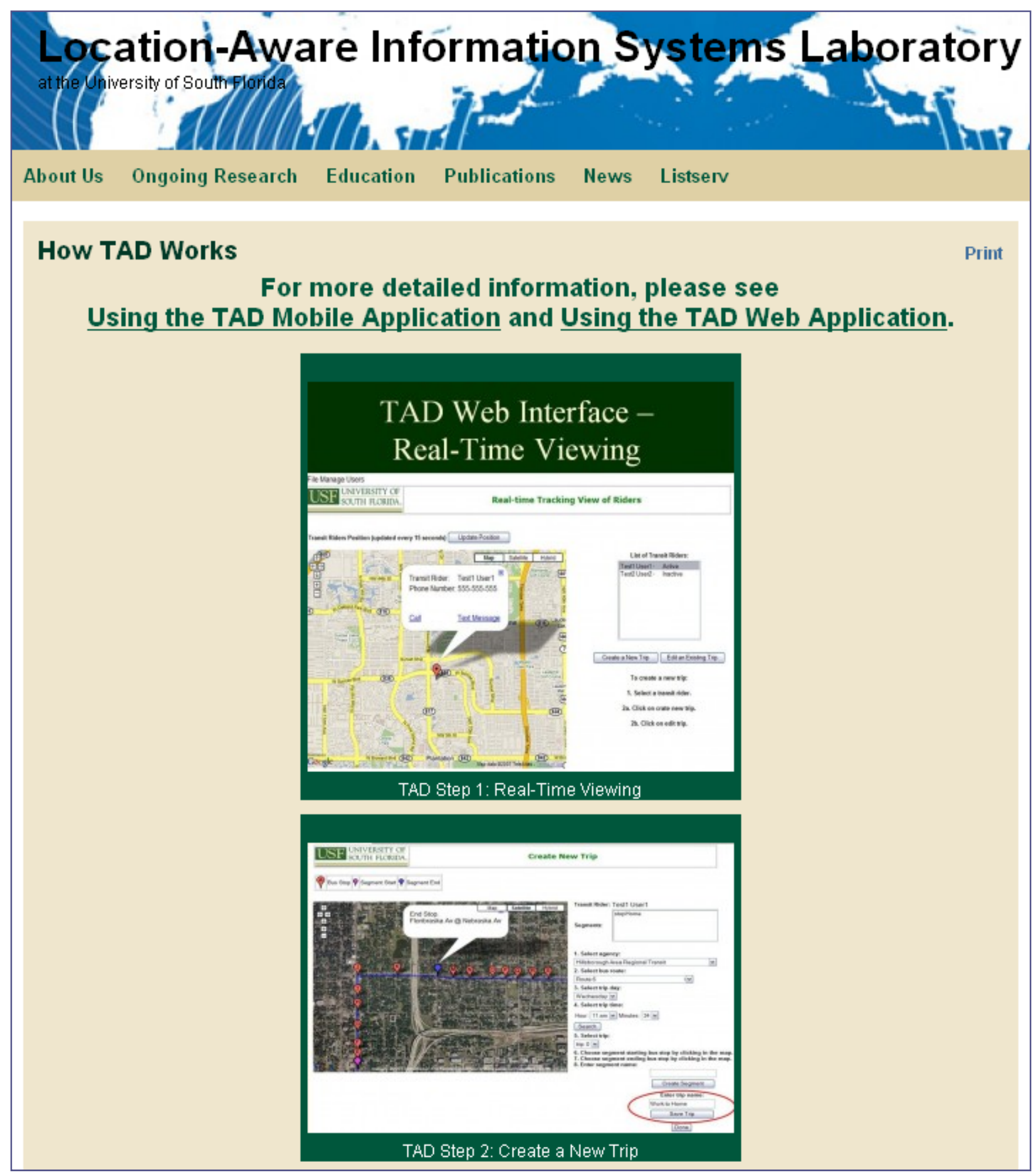

Figure 4 - Basic Online Information on How TAD Works 


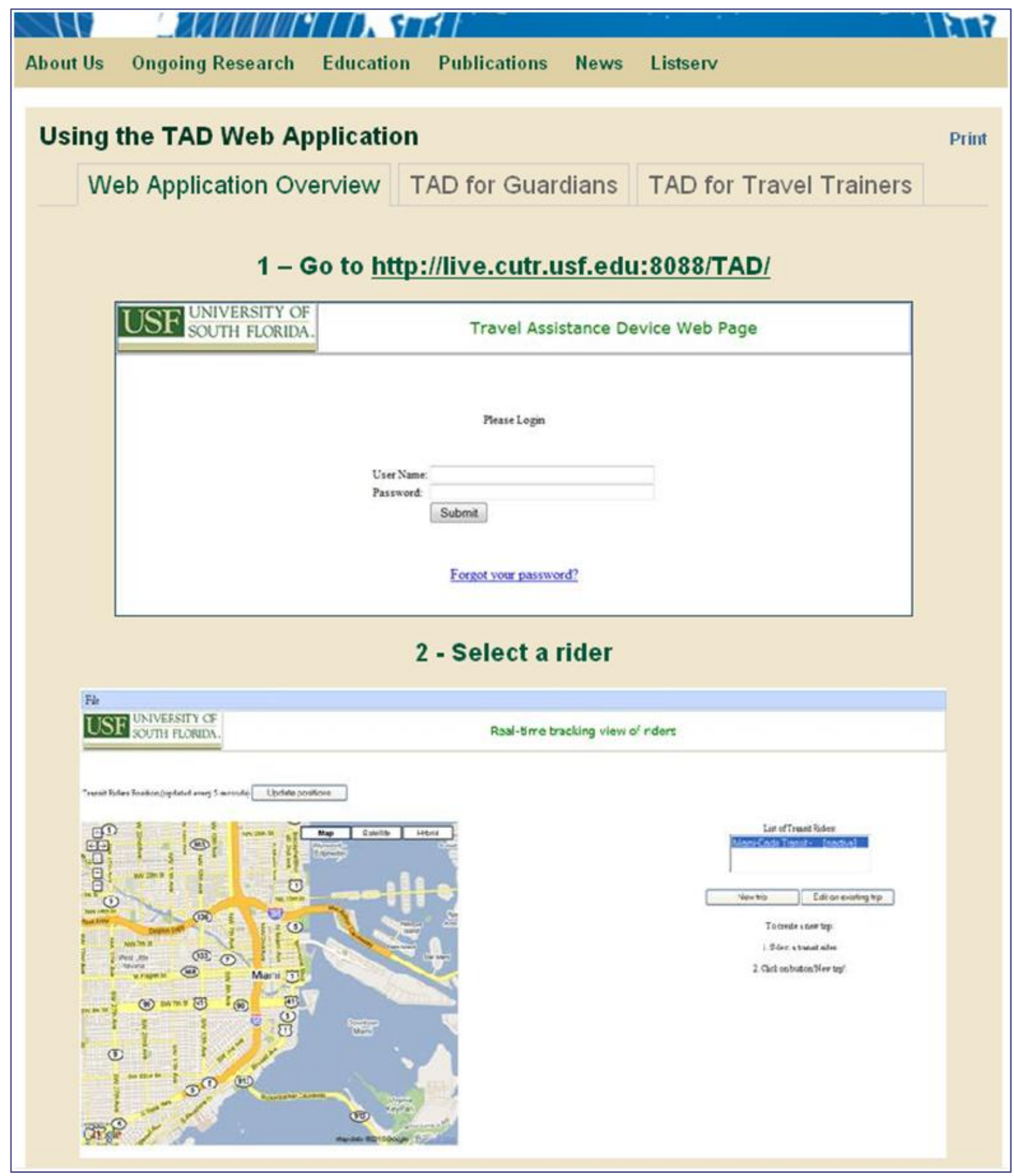

Figure 5 - Online Step-by-Step Instructions for Web Trip-Planning Tool 


\section{Using the TAD Mobile Application}

\section{Mobile Application Overview Starting TAD Updating TAD}

\section{$1-\log$ in to TAD}

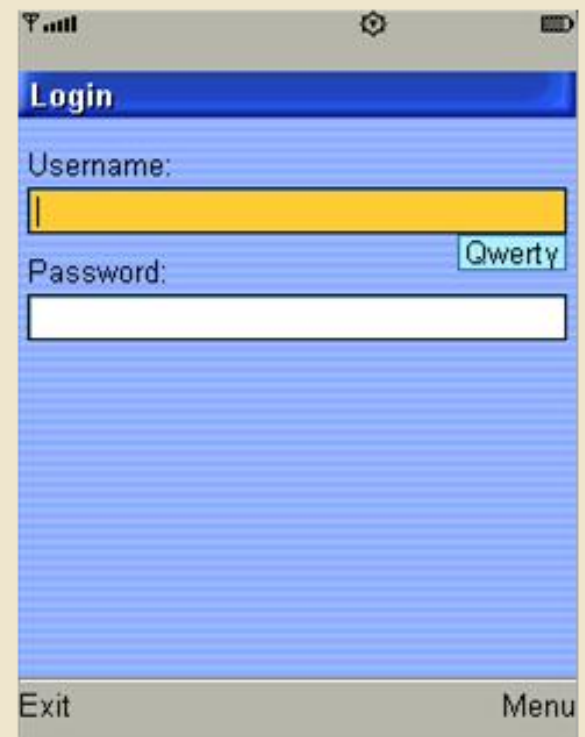

To reach this screen, you must first open the TAD application on the phone. Please see the instructions provided here.

Then type in your user name and password and select "Log in" from the menu.

Figure 6 - Online Step-by-Step Instructions for Using the

TAD Mobile Application

\section{Improving the TAD Server Configuration for Reliability and Availability}

One challenge identified in the initial TAD project phase was the difficulty with providing a stable TAD system that could be used by transit agencies to test TAD while the research team simultaneously debugged and improved it. The server-side components of TAD originally were hosted on a single-server machine, which contained both the Java Application Server software which communicated directly with the cell phone, as well as the database server software, which acted as the primary TAD data storage facility (Figure 7). 


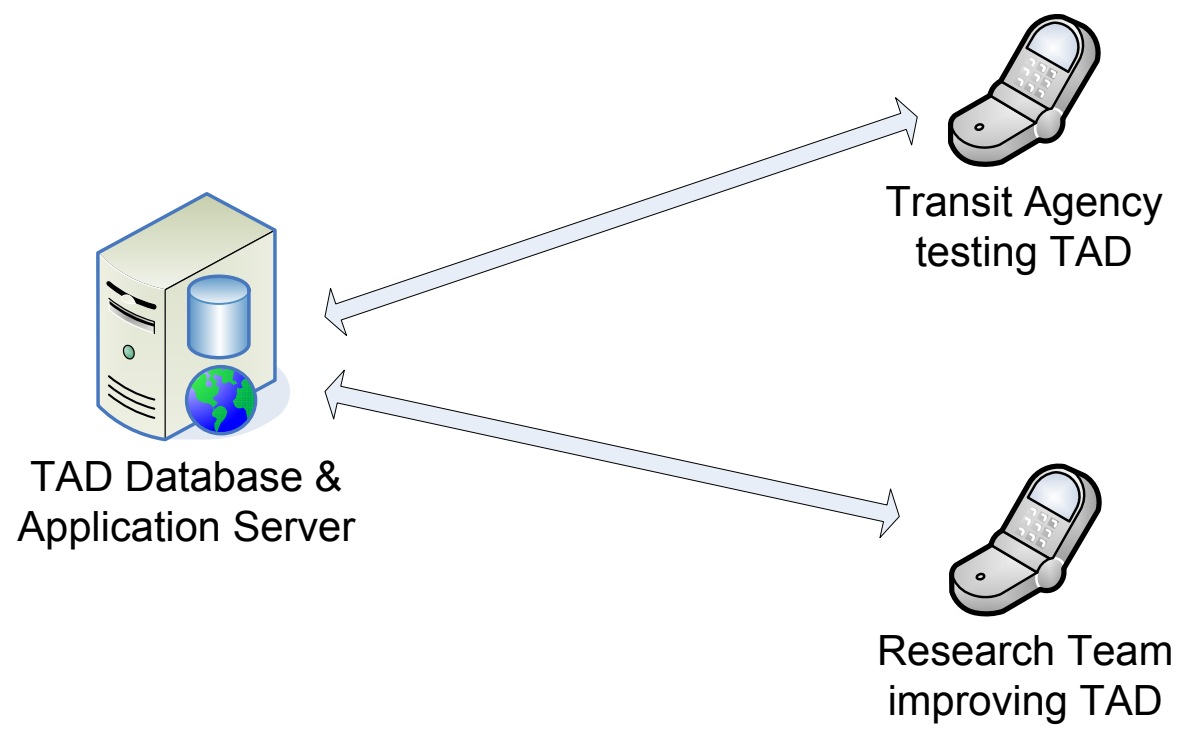

Figure 7 - Original Server Configuration for TAD

Since no changes could be made to the TAD software while the transit agency tested it, the testand-refine process had to be conducted in sequential fashion (i.e., testing by the agency and modifying TAD could not take place simultaneously). This inevitable delay in between testing by the transit agency, and troubleshooting by the research team caused field tests to take a long period of time and periodically resulted in interrupted service to the transit agency due to modifications made by the research team.

In preparation for TAD Deployment, the research team realized that if TAD testing were to take place by multiple agencies, then a sequential test/refine process would be time-prohibitive. The research team therefore redesigned the server configuration that supports TAD in order to separate the system into two parallel components: a development server system and a production server system (Figure 8). Under the TAD Deployment project, a new and improved Development/Production server configuration was implemented in order to increase the reliability and availability of TAD to transit agencies. 

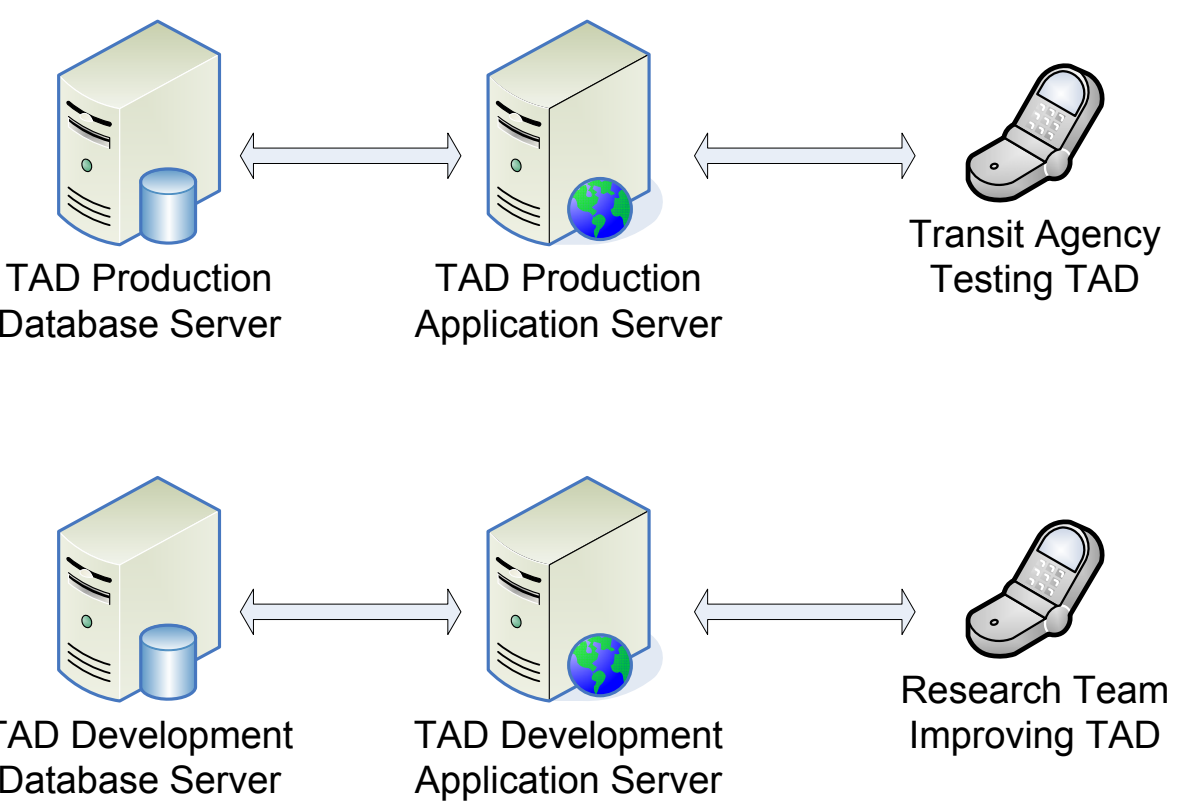

Figure 8 - Improved Development/Production Server Configuration

The production servers host the currently stable release of the TAD server-side software components that communicate directly with the cell phones being used by the transit agencies. This version of TAD software has been heavily tested by the research team and has proven itself to be stable. The production servers are running only the software vital to the execution of the mobile application, so the probability of the servers being unavailable due to problems with other software applications is reduced. By isolating each application and database server software on its own machine, performance of the system is improved and the adverse interaction between server software components is also reduced.

The research team is free to use the other set of servers, referred to as the development servers, for ongoing software development related to troubleshooting and improvements to the TAD system without interfering with the servers being used by the transit agencies. Changes are never made to the production system without first testing these changes on the development system. Once a new version of TAD has been thoroughly tested by the research team, this improved version of TAD can then be instantly moved over to the production servers for use by the transit agencies, reducing any interruption in service for the transit agencies.

New versions of the mobile software for cell phones are treated with a similar development/production design, and remote upgrades of the new TAD mobile software can be pushed to the cell phones being used by the transit agencies via an Over the Air (OTA) update process. Agencies then can update the TAD software on their cell phones by simply clicking on the link in a text message that is sent to the phone by the research team. This development/production design results in a more stable system offering greater reliability and availability allowing transit agencies to continue testing TAD while the research team troubleshoots problems and improves the TAD software system. 


\section{Adjusting the TAD System to Handle GTFS Data from New Agencies}

As mentioned earlier, in order to add the bus stop scheduling information of a transit agency to TAD, the transit agency must supply its bus stop information in a standard GTFS format. These data feeds are then imported into the TAD database by means of custom software application, the TAD Database Toolkit, created by the research team. The main functionalities of the toolkit are to add and/or update GTFS feed data from transit agencies, but it also can perform many other tasks related to the TAD database that are discussed in another section of this chapter.

GTFS was designed by Google and transit agencies as a standard for representing transit scheduling information and consists of several text files in CSV (comma-separated values) format. Transit agencies that use this format are asked to host a zip file named google_transit.zip on their Web site, which contains all the CSV files specified by GTFS. When the TAD Database Toolkit is instructed to update a particular agency's GTFS feed or add a new transit agency to the database, the toolkit will download the google_transit.zip file for that transit agency, unzip the file, and insert the data from each text file into temporary tables in the TAD database for further processing.

The toolkit then will begin processing each temporary table (which corresponds to a text file in the GTFS data) in three phases: Insert, Update, and Deactivate. The Insert phase adds new information to the database, such as new routes or new stop times. The Update phase updates information already in the database, such as modifying head-signs or moving bus stop locations. The Deactivate phase deactivates data such as old routes, trips no longer used, or removed bus stops. If data for a new transit agency are being added to the database, then the Update and Deactivate phases are skipped since there is no old data from that agency in the TAD database.

The GTFS, while very beneficial in regards to exchanging transit information, is not very specific when it comes to choosing data types, sizes, and meanings for the pieces of data it describes. Various interpretations of this standard can result in inconsistencies between the datasets from different transit agencies.

Data types and sizes are two very important factors when designing a table in a database. When designing a database table, each column's name, data type, size, whether it can be empty, and whether it should be unique must be specified. The research team made these initial design decisions in the first TAD project based on the GTFS data provided by HART. While the design decisions made early on worked well for HART's data, when the research team later imported the GTFS data of other transit agencies, several issues began to arise due to differences between HART's data and the data from other agencies. The research team was then faced with a choice - request the transit agencies to modify their GTFS data to match TAD's design expectations or modify TAD's design to accommodate the new data. The latter was chosen, and the most common issues faced and their solutions follow.

Data formatting was the most common issue encountered, in particular, the data types and field lengths. While HART normally used a number for bus stop IDs, route IDs, shape IDs, trip IDs, etc, PSTA and SCAT commonly had non-numeric characters, such as letters and symbols, in 
several types of IDs. TAD had expected these IDs to be integers, and the IDs were designed to flow through all the TAD components as integers, i.e., from the database to the Web application or from the database to the Web service to the mobile application. The fields identified with this issue were converted into a string format, and all references to these fields in the various TAD components were updated to handle the string format. For fields already in a string format, a common issue was the length of the string stored in the database. Several changes were made to the database in order to handle longer strings used by partner agencies.

An issue with formatting timestamps among transit agencies was identified and corrected. Most agencies listed their schedules with the times including a leading zero, e.g., 09:00. A few agencies listed the times with a leading space instead of a leading zero, e.g., 9:00. The leading space caused issues with queries that searched for trips between two times, so a fix was created to replace the leading space with a leading zero upon inserting the data into the TAD database.

Many of the technical issues encountered while testing with transit agencies involved data conversion issues that propagated through the TAD system after resolving issues with the GTFS data imports. Each of these issues was apparent only after attempting to import data from a new transit agency into the TAD system. All applications retrieving GTFS feeds from agencies will have to deal with these data formatting inconsistencies, and will need to modify their import tools and system to handle these differences.

\section{Creating Off-Route Notifications Based on Transit Route Spatial Information}

When using the TAD mobile application while traveling on the bus, the rider's real-time position is constantly monitored by server-side software to make sure he/she is still on the correct path to his/her destination. The problem that arose from these checks in the original TAD project was that the constructed path that TAD uses as the bus route is more of a connect-the-dots representation by drawing a path from bus stop to bus stop, rather than the exact route a bus travels. Figure 9 illustrates the improvements to the spatial data representation of a bus path. A connect-the-dots approach was necessary in the original system design since the HART GTFS feed at that time did not include any spatial information about the actual travel path of the bus, only the bus stops that the bus visited. Since the bus would travel outside of this line when traveling around corners, the system would trigger a false-positive for off-route detection and send text messages indicating that the rider might be lost, when he/she was, in fact, on the

correct bus. The top image in Figure 9 shows an assumed straight path between bus stops, and the bottom image shows the actual path traveled by the bus. 


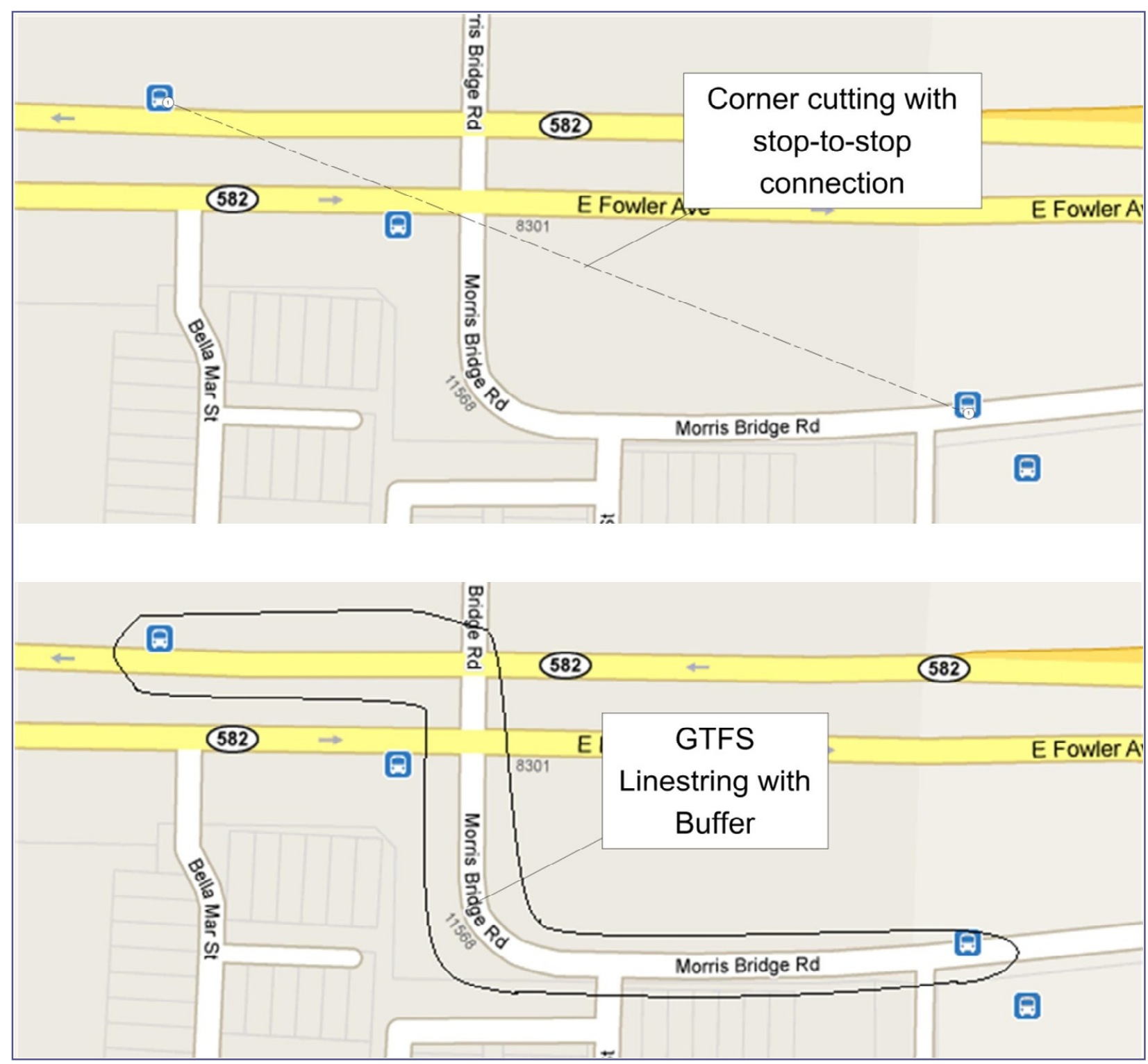

Figure 9 - Straight Line vs. Actual Travel Path between Bus Stops

While there is a shape file in the GTFS that allows the transit agency to represent the true travel path of a bus, this file is optional and was not populated by HART during the original TAD project.

To alleviate this problem, a method was created to automatically generate a spatial data representation of transit agency routes using the shape file data provided in an agency's GTFS data. HART began including this information in its GTFS feed shortly after the original TAD project concluded.

The shape file information is extracted from the GTFS feed as part of the TAD Toolkit data import process. The shape data are then stored in the TAD database where they easily can be 
accessed by the system. Code was then written to parse the given information into geometry objects recognizable by a spatial database. The process involved turning longitude and latitude data into geometric points, which were then strung together into linestrings representing a route. A buffer was drawn around the linestrings to assure no unwanted notices were given by the destination checks, since the buffered linestring should more than adequately cover the actual travel path of the bus, including potential GPS errors from the cell phone.

The geometric data obtained through processing the shape files are inserted into a spatial database with each element corresponding to a unique shape ID. Figure 10 graphically displays the process.

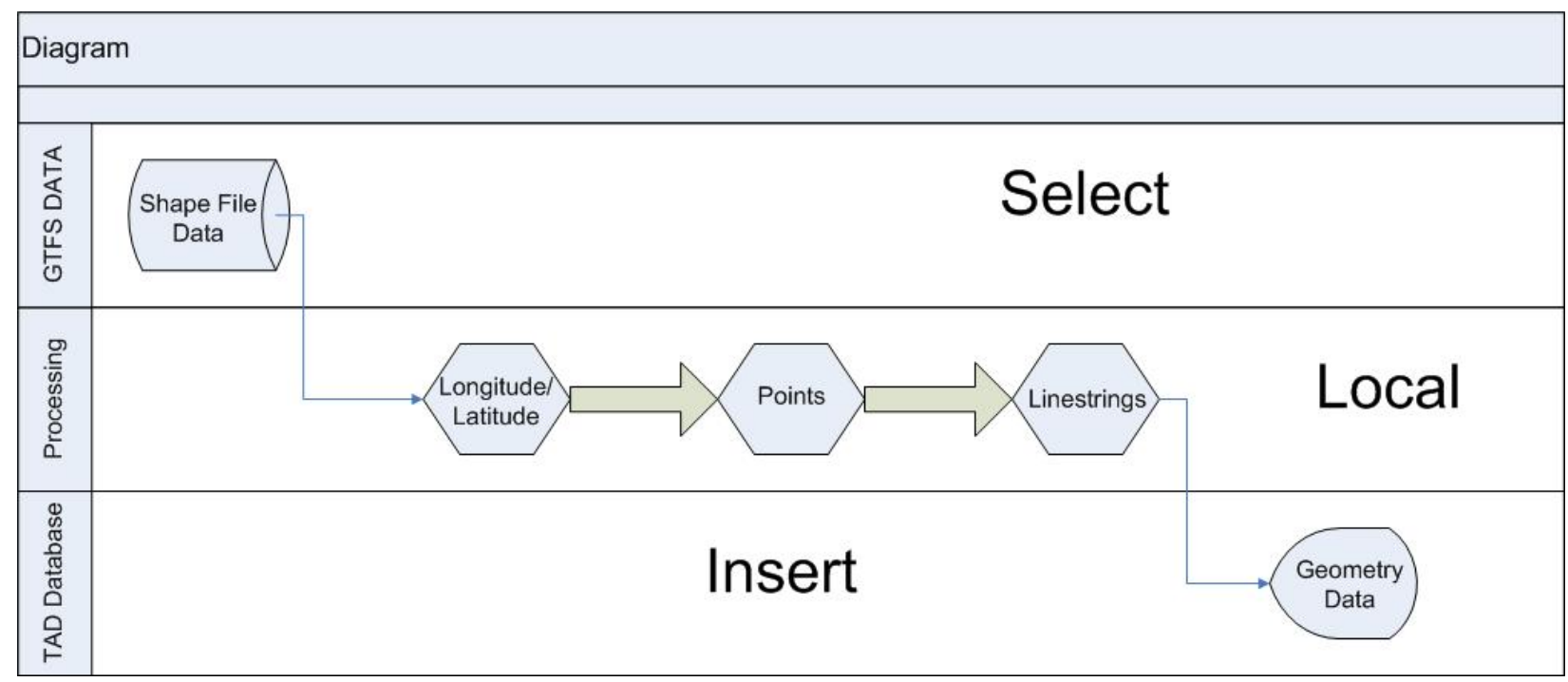

Figure 10 - Process for Transforming Shape Data into Geometry Data Used in Off-Route Notifications

Once the geometric data were created, they were then used to identify when a transit rider has deviated off the route and send the appropriate notification.

\section{Resolving TAD System Issues from Integrating Data from Multiple Agencies}

Since the original TAD project used transit data from one agency (HART), the TAD server, Web site, and mobile software had never been tested with more than one agency's data prior to the TAD Deployment project. Since TAD will maintain many transit agencies' data simultaneously in the same database, a sophisticated system of unique identifiers must be used so that data from each agency can be properly referenced. The GTFS format requires that only stop, route, and trip IDs be dataset unique (i.e., unique within a single GTFS zip file), but cannot require that they be unique among agencies (since each agency does not have knowledge of all the other 
agencies identifiers). Therefore, TAD must maintain its own system of unique identifiers in case the identifiers from agencies' GTFS data collide with the same values.

During testing of the TAD system by the research team in the development environment prior to deployment to transit agencies, it was discovered that nearly half of the Pull the Cord Now! prompts were not being fired. It was also noted that on the occasions where the prompts were missed, the distance from the second to last stop was an abnormally large number. After investigating the issue further, it became apparent that the cause of the missed prompts was due to an issue with bus stop IDs.

Due to the addition of multiple transit agencies, a piece of code that retrieves the locations of the second to last stop (the stop in which TAD uses to compare the phone's current location to when deciding to give the alerts) was not specifying which transit agency the stop belongs to. This means that if two bus stops have the same ID across multiple transit agencies, the information to the wrong stop may be returned to the phone.

This issue was not apparent in the production environment at the time as the issue only occurs when GTFS data feeds from multiple agencies exist in the database with the same bus stop IDs. For example, both PSTA and HART may have a bus stop with an ID of 20. As TAD was configured to retrieve information on bus stop 20 without respect to which transit agency is being requested, the wrong bus stop may be used in the algorithm that determines when to provide the alerts. A small code change quickly resolved the issue and since then very few further prompts were missed during internal testing by the research team.

Another issue was discovered by the research team prior to deployment to the transit agencies. During multi-segment trips, the phone screen would freeze up and the screen would continue to read "Confirming... Please wait..." when it should have displayed tracking screen for the next segment. The team eventually resolved the issue which stemmed from an earlier enhancement that aimed to prevent the accidental pressing of the phone's buttons.

\section{Integrating a Bus Stop Management Tool with the TAD Web Site}

To provide transit agencies and other appropriate individuals with the ability to update or correct the geographic locations of bus stops, a bus stop management tool was developed and integrated into the TAD Web application. It is not uncommon for a transit agency to have an outdated or inaccurate inventory of bus stop coordinates, since, in certain cases, the transit agency may not need highly accurate knowledge of where its bus stops are located in order to provide basic transit service. However, TAD, as well as other more recent transit technologies such as AVL used for schedule adherence, requires highly accurate (i.e., error of less than $5^{0}$ meters) knowledge of the bus stop locations. This tool aims to overcome this deficiency by allowing manual updates to bus stop locations to ensure the TAD mobile application will function properly along the transit agency's routes.

The bus stop management tool in the TAD Web application for editing bus stop locations is shown in Figure 11. The user must first enter the search parameters in the bus stop form in the 
upper right to search for the desired trip. After filling out the form and clicking the Load trips button, the user can select the correct trip from the trip dropdown list, click the Open trip button, and the bus stop markers will automatically load on the Google Map display. The user can then drag the markers to the proper locations on the map. A blue line always shows the place of the original coordinates. A list of modified coordinates is displayed on the screen and when the user is finished, they can click the Save coordinates button to submit the new coordinates to the database. It is expected that only authorized personnel from the transit agency, such as travel trainers or other individuals who would have first-hand knowledge of real bus stop locations, and TAD system administrators would have access to the bus stop management tool.

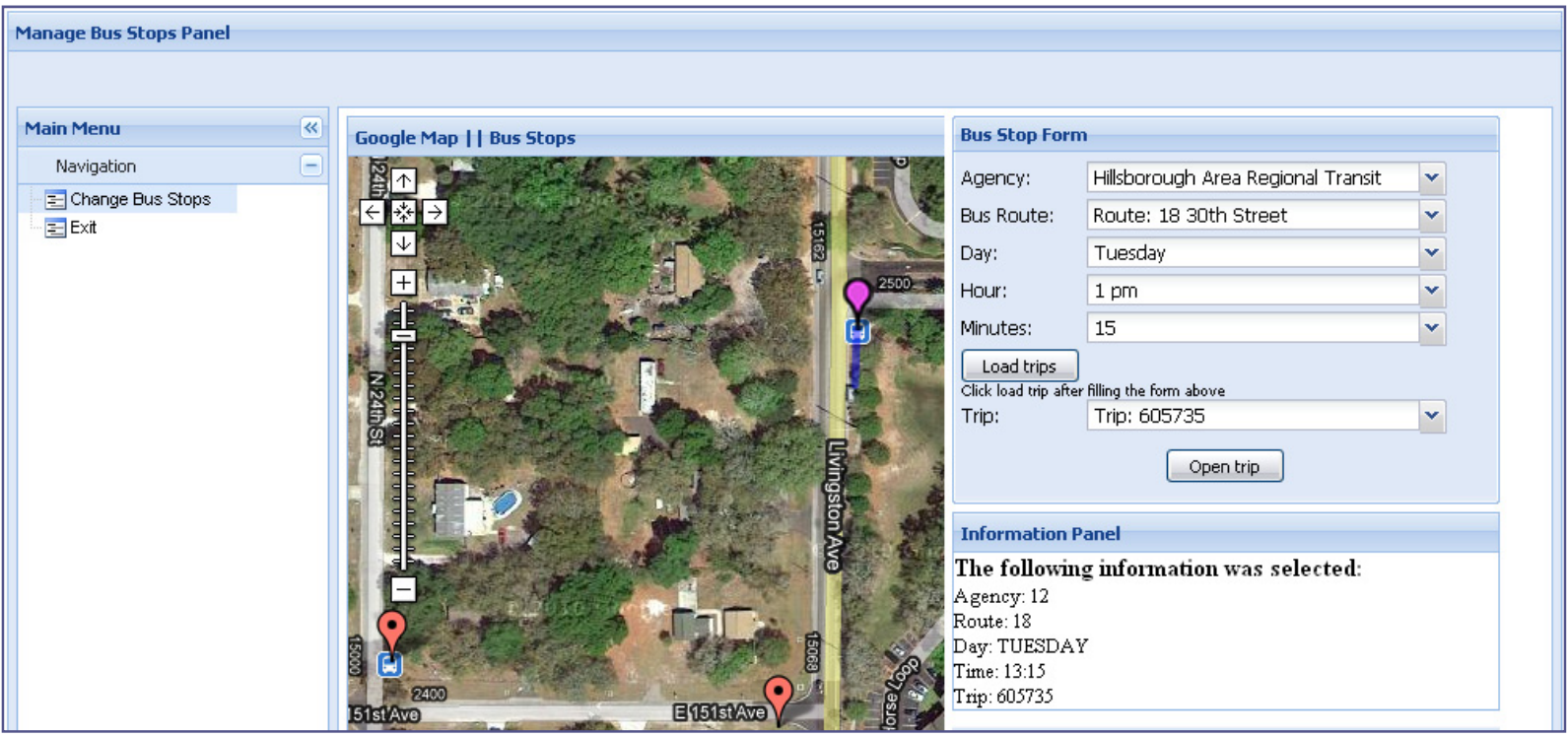

Figure 11 - Bus Stop Management Tool

\section{Maintaining User Data Consistency after Transit Agency GTFS Data Updates}

As discussed earlier, TAD uses data provided by transit agencies and formatted in the GTFS format to update the TAD system for changes in bus routes, stops, and schedules made by the transit agency.

In the GTFS, a trip is defined as the order of visitation of a number of bus stops on a route at a particular time and therefore is an important piece of data that links unique routes, stops, and schedules (see Figure 12). After a user plans a trip using the TAD Web site, the TAD system saves the correct GTFS trip ID as a unique identifier to determine the order of visitation of stops and alert the rider to exit the vehicle at the appropriate time for all future trips. As discussed later, this order of visitation information is required to determine the locations of the Get Ready 
and Pull the Cord Now! alerts that are delivered to the user. Figure 13 shows the fields which capture user input that is translated to a trip ID.

\begin{tabular}{|c|}
\hline Trips \\
\hline +trip_id \\
route_id \\
service_id \\
block_id \\
shape_id \\
\hline
\end{tabular}

Figure 12 - Data Structure of TAD Trips Database Table

When TAD was originally developed, the research team assumed that these trip IDs would remain consistent across new GTFS data updates from the agency. However, the transit scheduling software (e.g., Trapeze, HASTUS) that transit agencies use to output their data in the GTFS format changes trip IDs between each system update. So, while trip IDs in each update are dataset unique (as required by the GTFS format), trip IDs are not consistent or unique across system updates. This detail is not noted in the current GTFS. Therefore, the original system TAD could not ensure that a particular trip (and therefore route, stop, and schedule) still exists after a system update by relying solely on the trip ID, since this ID could change when an update is executed. As a result, TAD Web site user would need to re-enter each of their planned trips after an update, which caused unnecessary work for TAD users and is not acceptable for widespread use of TAD.

Figure 12 shows the structure of the Trips table. The field trip_id is an integer value that uniquely identifies a trip. Thus, there are no two different trips with the same trip_id within a single GTFS feed. Nevertheless, trips are not necessarily consistent across updates as discussed above. This implies that, for instance, a certain trip whose trip_id = 1 in the update of March 2009 might change to trip_id = 2 after the update of April 2009. Additionally, a trip ID could actually be a string of alphanumeric characters and is not necessarily a number.

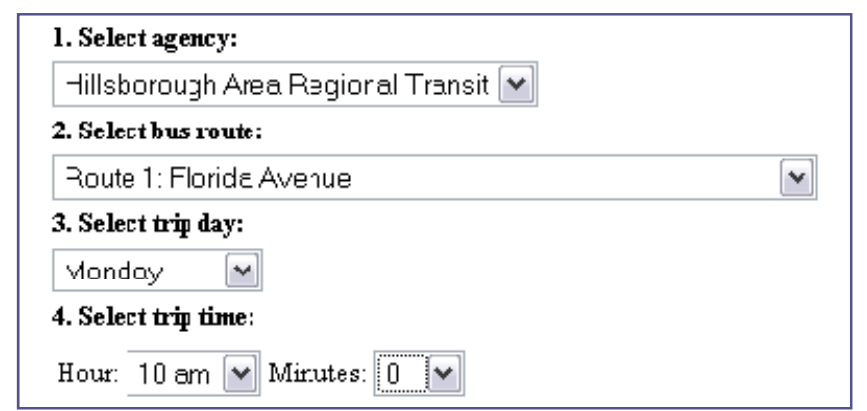

Figure 13 - Required Input for Creating a New Trip on TAD Web Site 
Since segments, services, and all the data that TAD stores should correspond to a unique trip, a new Trip Coherence algorithm was designed under this project to uniquely identify trips, even if their trip_id varies along updates.

\section{Data Preparation}

In preparation for the Trip Coherence algorithm, whenever the user creates a new trip on the TAD Web site, additional information is now saved: the second to last bus stop id (SLID, used by the mobile application to alert the user of an upcoming stop), the ID of the transit agency, the route ID, the day of the week, and the time. All these fields are provided by the user of the TAD Web site in the Create New Trip screen when a trip is planned on the TAD Web site (Figure 13), except the SLID, which is calculated from the trip information in the table Stop_Times. For individuals with cognitive disabilities, a travel trainer or caretaker will most likely be the person planning trips for the transit rider via the TAD Web site. Figure 14 shows the data structure of the table containing information for a planned trip.

\begin{tabular}{|c|}
\hline Stop_Times \\
\hline trip_id \\
arrival_time \\
departure_time \\
stop_id \\
stop_sequence \\
pickup_type \\
drop_off_type \\
shape_dist_traveled
\end{tabular}

Figure 14 - Data Structure of Stop-Times

The Stop_Times table is necessary to link every trip at a determined time to the set of stops that belong to it. The order in which the stops should be visited by the bus is also indicated by the field stop_sequence. This field is a consecutive integer number such that a greater value of stop_sequence implies that the bus stop should be visited later.

Therefore, to calculate the SLID, the value of stop_sequence for the end stop should first be determined. Then, some extra analysis is required to correctly choose the SLID. If the set of bus stops within a trip is such that every bus stop should be visited exactly once, there is a trivial solution to compute SLID: if the end stop has stop_sequence $=n$, then choose as second to last stop the bus stop whose stop_sequence is $\mathrm{n}-1$.

However, some routes have a different structure, so that the same stop could be visited twice along the same trip. Figure 15 illustrates an example of a route with an intersection point. Notice that, in this case, the stop marked as red has two different sequences: 21 and 28. If the user selects that particular stop as the end stop of a segment, the second to last stop could be either 20 or 27 (marked as purple). 


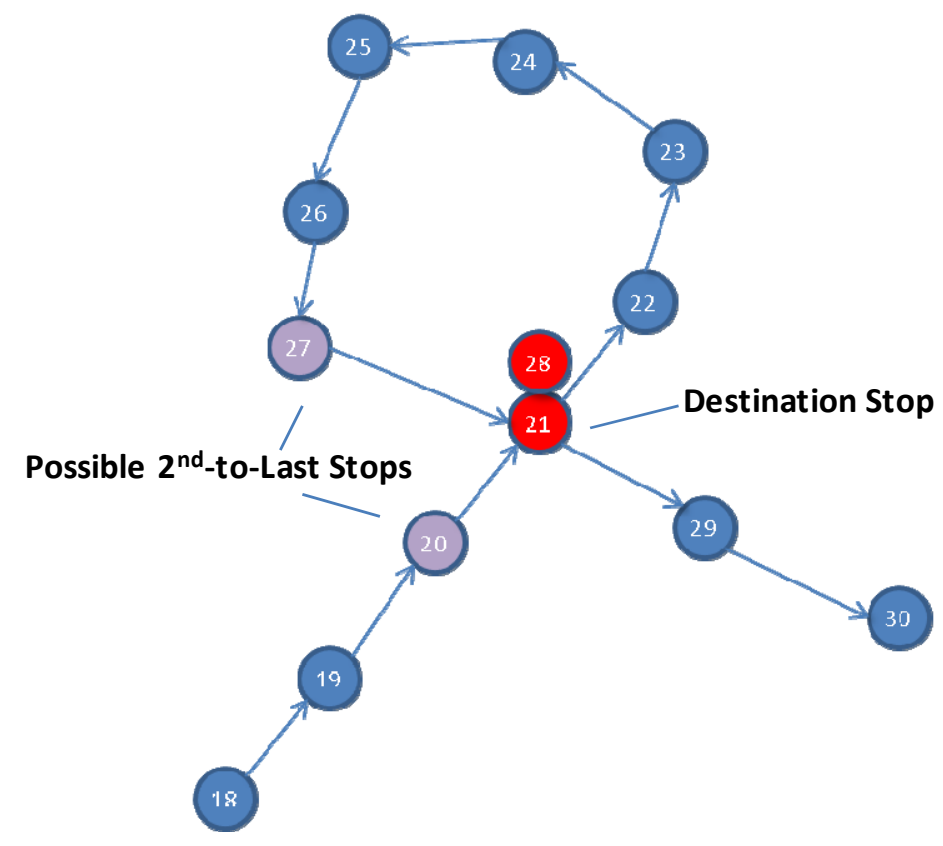

Figure 15 - A Single Intersection on a Route Gives Multiple Sequence IDs

This issue could become even more challenging if the start stop is also an intersection, as shown in Figure 16. TAD provides flexibility to handle each of these situations and properly identify the second-to-last stop for use by the TAD mobile application.

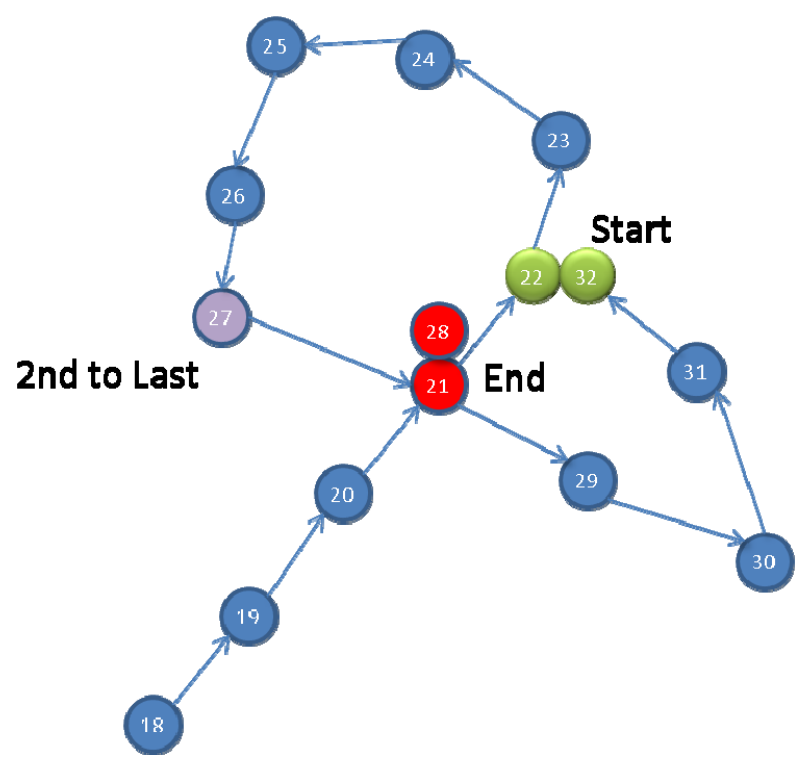

Figure 16 - Multiple Intersections on a Route Complicate IDs Further 
The proposed solution to determine the second to last stop is as follows:

1. Determine start stop sequence: From the two start stop sequences, choose the lowest one. This is 22 in the example of Figure 16.

2. Determine end stop sequence: From the two end stop sequences, choose the lowest one. If it is lower than the start stop sequence already chosen in step 1, then the end stop sequence will be the greatest one. In the presented example, it is 28 .

3. Determine second to last stop sequence: Finally, the second to last stop sequence is obtained by subtracting one unit to the end stop sequence just found in step 2. This is $28-1=27$. Once the stop_sequence is known for the second to last stop, the SLSID can be easily calculated by a cross-table query, and this value is stored in the database.

\section{Trip Coherence Algorithm}

Once the proper information is saved upon the user planning a trip, the Trip Coherence algorithm can then be executed upon each GTFS update within the TAD Database Toolkit. In this way, all the previous trips become inactive and the algorithm finds an equivalent trip for each one of the inactive trips and automatically ties each of these new GTFS trips to the user's trips.

The update process is, therefore, transparent to the end users of TAD as long as the agency has not made any changes to its operations that affect the user's trip. If the transit agency has made changes that affect a user's trip (i.e., if there are no matches in the new GTFS data for an existing trip), the user is notified via an email message. Once notified, a user would need to re-plan the trip using the TAD Web site and determine if his/her destination is still reachable, given the transit agency route changes.

The Trip Coherence algorithm begins by deactivating all the old GTFS trips for that transit agency in the TAD database. This implies that all user-created segments generated from the TAD Web site become inactive as well, since they will be pointing to non-active GTFS trips. Now, a new GTFS trip must be found that matches the old, now inactive, GTFS trip.

This problem is now defined in more precise notation: for every user-created segment $\mathrm{G}$ that is matched to an inactive trip $\mathrm{T}$, the goal is to find an active trip $\mathrm{T}$ ' such that $\mathrm{T}$ and $\mathrm{T}$ ' are equivalent. Thus, $\mathrm{T}$ and $\mathrm{T}$ ' belong to the same route, same transit agency, and the same set of associated stops in the same order of visitation.

Next, an efficient method to compare the stop sets $\mathrm{S}$ and $\mathrm{S}$ ' that are associated to T and T' respectively must be implemented. This is because every inactive trip has to be compared, in the worst case, with all other trips. Since a single agency can have thousands of trips in its GTFS data, this could take $\mathrm{O}\left(\mathrm{n}^{2}\right)$ time, where $\mathrm{n}$ is the number of trips in the agency's GTFS data.

Several simple solutions can be considered

- Comparing starting and ending stops of both S and S';

- Comparing the number of stops (sizes of $S$ and $S^{\prime}$ ); 
- Verifying that starting stop, second-to-last stop, and ending stop appear in the same order in both S and S'.

However, the previous methods do not guarantee that $S=S^{\prime}$. In fact, two different trips may have the same starting and ending stops, or the same number of stops. Also, two different trips may have the same starting, ending and second-to-last stops, but the route itself may have changed.

\section{Comparing Trips Efficiently}

The proposed solution is to create a simple hashing function $H(T)$ that summarizes the set of stops of a trip, also considering the stop sequence, $\mathrm{T}$, into an integer number. This function is defined as follows:

$$
H(T)=\sum_{i=1}^{n} i \operatorname{dstop}(i) * \text { stopsequence }(i)
$$

Where $\mathrm{n}$ is the number of stops of the trip $\mathrm{T}$.

If two stop sets are different, they will have different hashcodes. Therefore, the set of stops of T does not need to be compared with all the sets of stops of all the trips in the system. Instead, we only need to compare $\mathrm{T}$ with trips with the same hashcode.

\section{Implementation Considerations}

To minimize the number of database operations, all the trip hashcodes are calculated and stored into the database. For this purpose, a new numeric field haschode has been added to the table tblTrips, which stores user-created trips from the TAD Web site. The SQL function

TRIP_HASHCODE was created to compute the hashcode of a given trip specified by its tripkey.

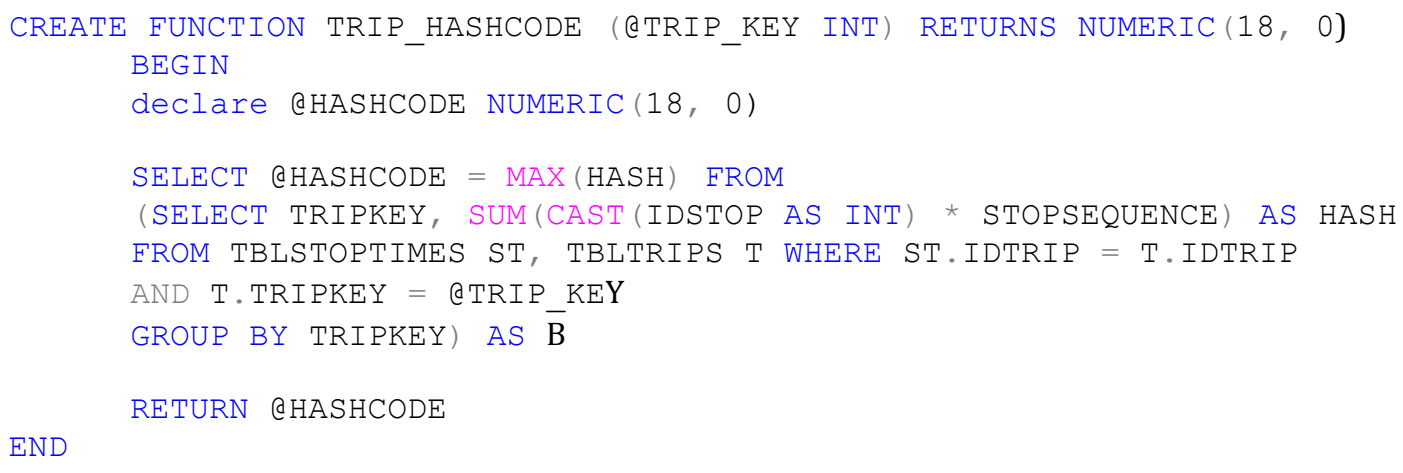

Even though this is the most natural way to calculate hashcodes, the tests show that this 
function takes about six seconds to calculate the hashcode of one single trip. This can be timeprohibitive in real operation, since for a GTFS feed with 15,000 trips the process would take no less than 25 hours.

Therefore, another approach was developed without using the previously defined function. Indeed, all the hashcodes can be computed in roughly seven seconds by using

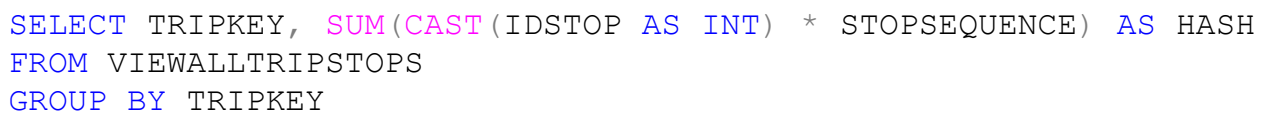

Now, the following SQL command stores the result of the previous query into the field hashcode of table tblTrips:

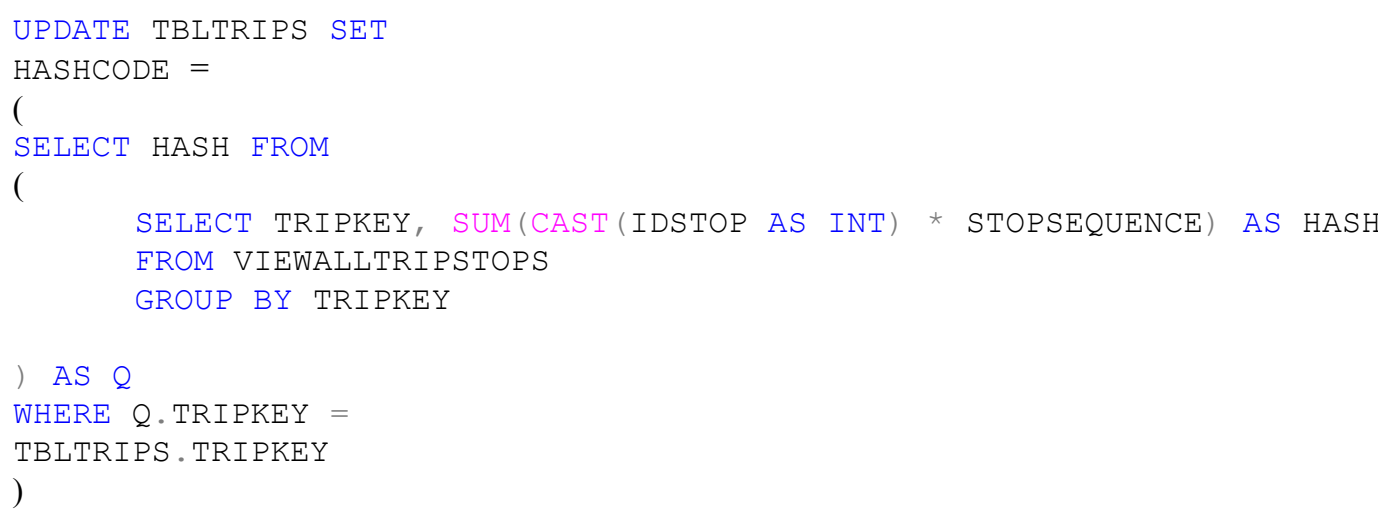

The Final Algorithm

The final algorithm is summarized as follows:

For every record $\mathrm{R}$ in the table tblTripSegment whose tripkey corresponds to an inactive trip, try to find an equivalent active trip T', and then update the tripkey of $\mathrm{R}$ with the value of T'.tripkey.

The detailed steps of the algorithm are as follows:

1) Find the set I of inactive trips (the old ones) that are used by current user-created segments.

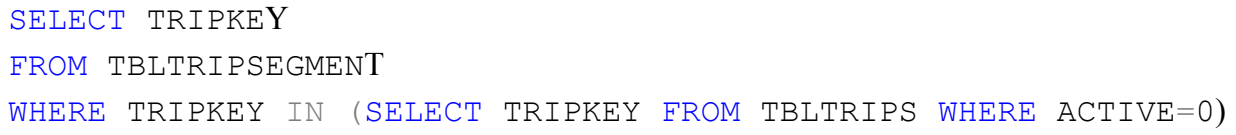

2) To find the stop sets of all inactive trips, a new view, view InactiveTripStops ,was created. The new view is similar to viewTripStops, but the difference is that viewTripStops only considers active trips. viewInactiveTripStops is as follows:

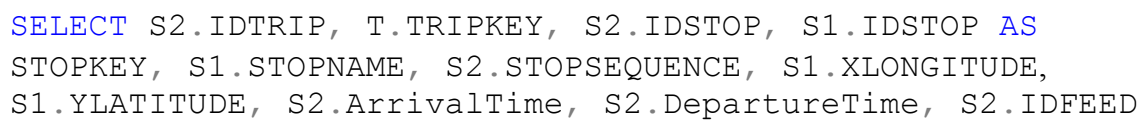


FROM dbo.tbISTOP AS S1 INNER JOIN dbo.tbISTOPTIMES AS S2 ON

S2.IDSTOP = S1.IDSTOPTRAGENCY AND S1.IDFEED = S2.IDFEED AND

$\mathrm{S} 1 . \mathrm{ACTIVE}=\mathrm{S} 2$. Active INNER JOIN dbo.tblTrips AS $\mathrm{T}$ ON

$\mathrm{S} 2 \cdot$ IDTRIP $=\mathrm{T} \cdot$ IDTRIP AND S2.IDFEED $=\mathrm{T} \cdot$ IDFEED

WHERE (S1.ACTIVE = 'FALSE')

3) Similarly, the view viewAllTripStops, which is the union of viewTripStops and viewTripStops, contains the stop sets of all trips (both active and inactive).

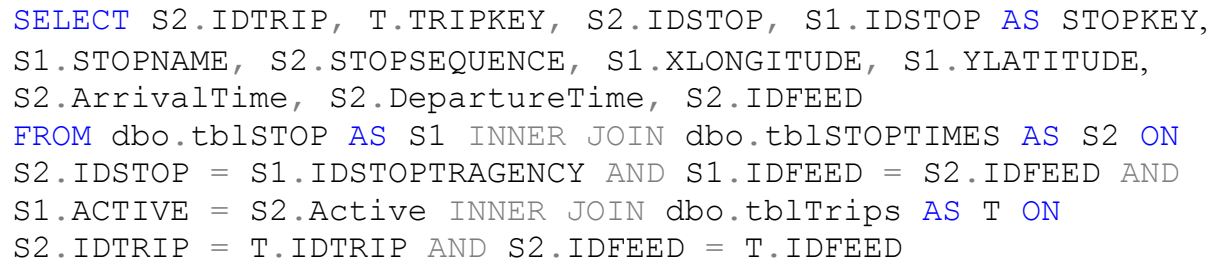

4) For every inactive trip $\mathrm{T}$ in I (found in step 1), do the following:

a. Get the hashcode $H(T)$ of trip $\mathrm{T}$.

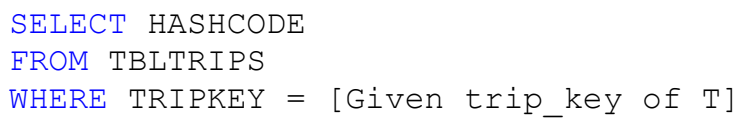

b. Search for an active trip T' such that $H(T)=H\left(T^{\prime}\right)$. (In other words, search for a trip with the same hashcode).

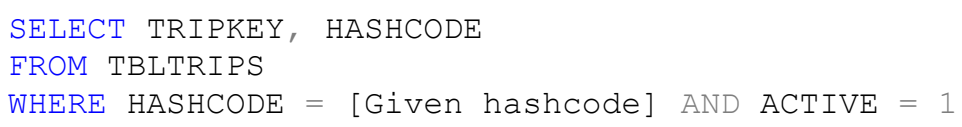

c. If T' is not null, then check that the sets of stops $S$ and $S^{\prime}$ (for $T$ and T') are equal. This can be done by checking that $S_{\text {union }} S^{\prime}=S$ (considering the stop sequence and the time). Using SQL operations, this is efficiently accomplished by checking if the numbers of rows of the following two queries are equal:

i. The stops in $\mathrm{S}$

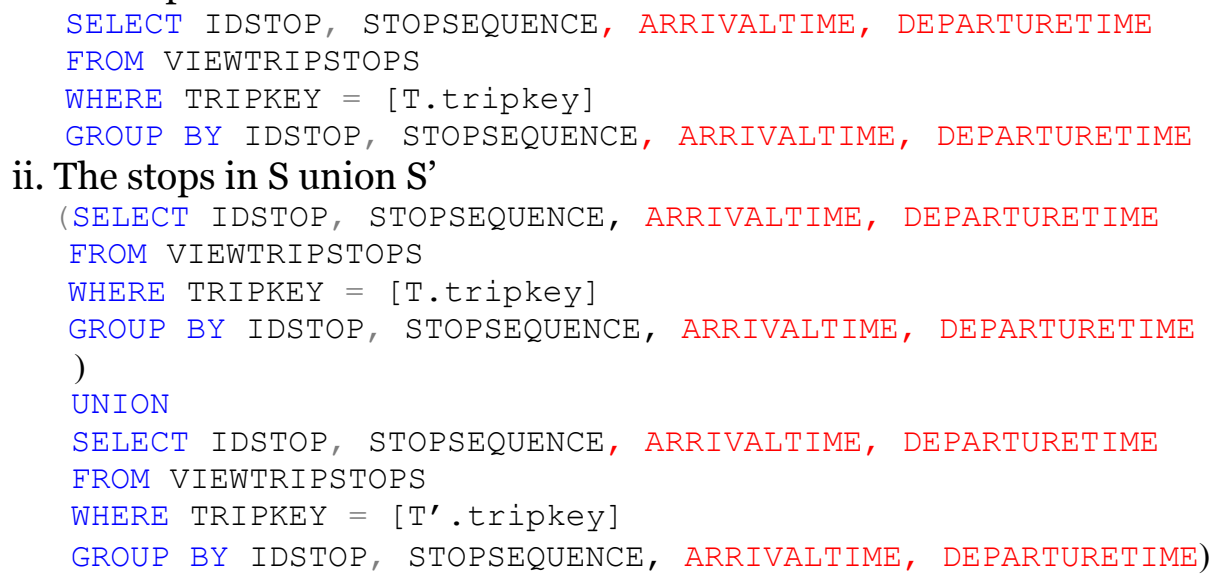


d. Update the tripkey of the user-created segment:

UPDATE TBLTRIPSEGMENT SET TRIPKEY = [T'.TRIPKEY]

WHERE TRIPKEY $=[$ T.TRIPKEY $]$

\section{Memory Optimization for Tracking Screen in the TAD Trip Planning Web Site}

The tracking screen of the TAD Web page displays markers that represent the real-time locations of transit riders and buses. Since the number of buses and/or transit riders could be arbitrarily large, TAD now incorporates a new approach for drawing markers over the map.

In the previous system design, every time the rider and bus positions were updated, all the markers on the map were removed and created again with the most recent coordinates. However, browsers such as Internet Explorer and Mozilla Firefox maintain memory references to the old markers (i.e., old markers are not removed from system memory). This implies that after a certain amount of time, the TAD Web page will consume a high amount of memory and in some cases, it could even block the Operating System (e.g., Windows) or significantly degrade the performance of the computer.

In the new design, the Web page application stores a unique list of markers that contains the position of every marker. Rather than creating new markers when updating, the TAD Web page queries the new coordinates from the database and dynamically updates the position of all markers. In this manner, the memory requirements of TAD Web page remain constant over the life cycle of the application and do not increase over time.

\section{Enhancing the Appearance of the TAD Mobile Application}

The new user interface of the TAD mobile application includes more intuitive and appealing icons to indicate the GPS status. Figure 17 illustrates the new displayed icon when GPS is available, and Figure 18 shows the icon displayed when the GPS signal is not strong enough for TAD to obtain location information. The main screen now includes more visible labels. In the very top title bar, the trip name is displayed - USF to Wal-Mart. Immediately below, the route information appears. The transit rider can be aware of which bus he/she has to board by viewing the route information (e.g., Route Five in the case of Figure 18). Finally, at the very bottom of the screen, the distance to the destination stop is shown. 


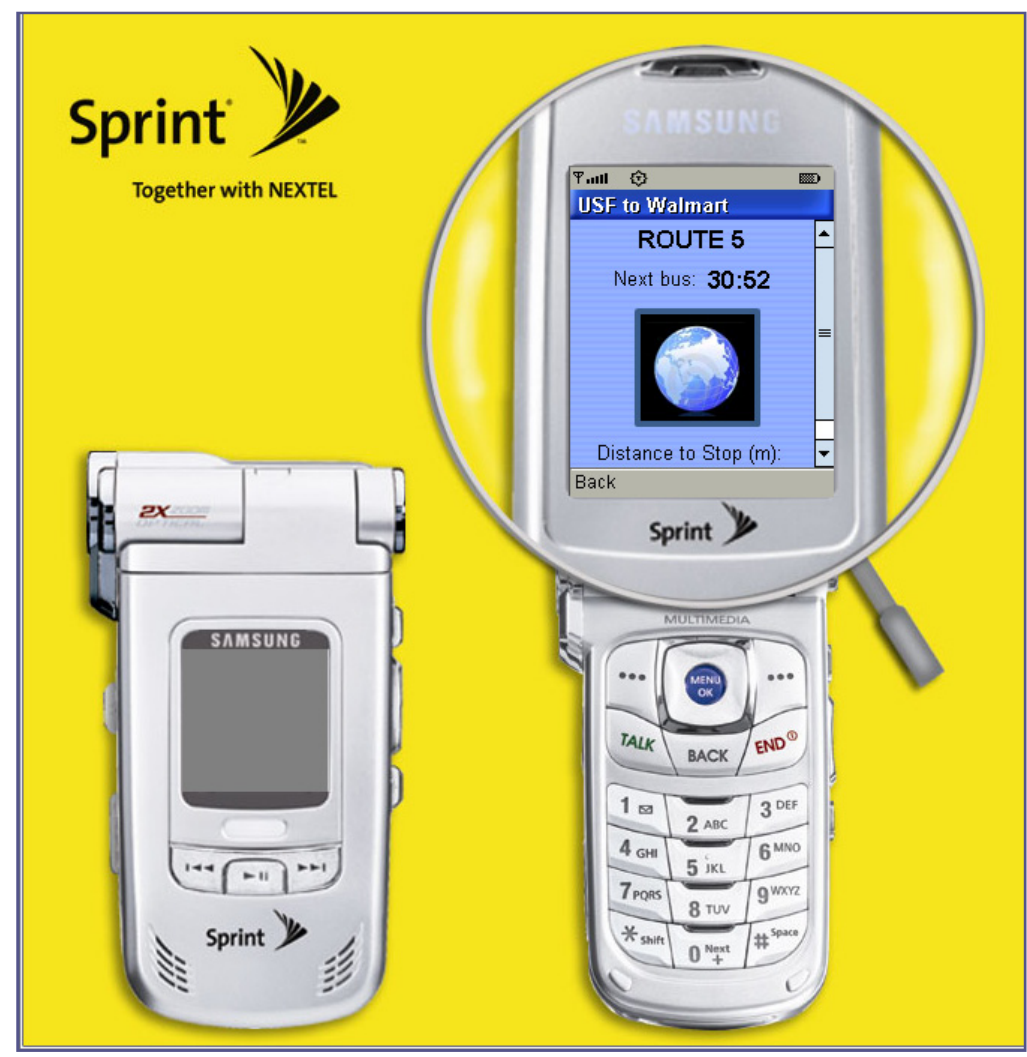

Figure 17 - New Appearance When GPS is Available 


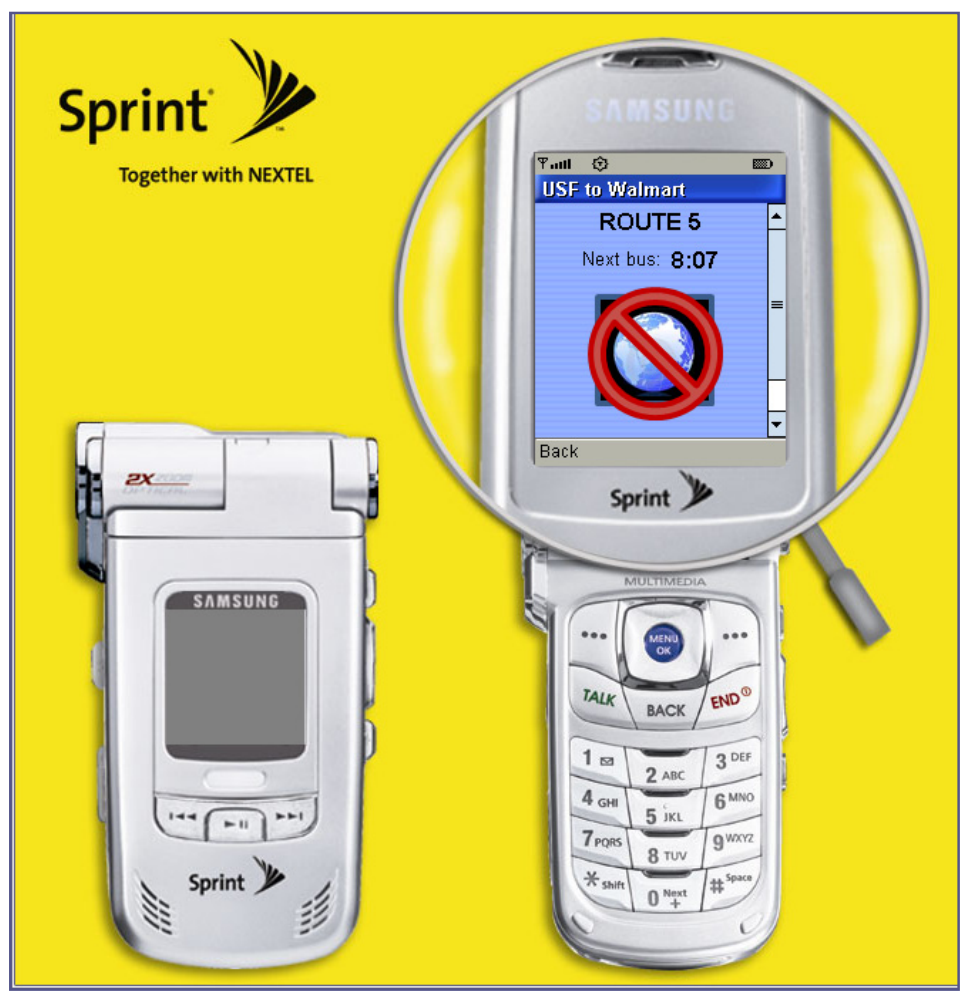

Figure 18 - New Appearance When GPS is Unavailable

\section{Improving the TAD Database Toolkit User Interface \& Capabilities}

As mentioned earlier, the TAD Database Toolkit is an administrative tool for maintaining and updating the TAD system. Its main function is to import or update the GTFS feed data from the different agencies. Other important features include generating XML output files for use with the phone emulator for debugging purposes, building KML files for viewing trips in Google Earth, setting up a new transit agency in the TAD system, modifying bus stop locations manually, and using an Excel file to update bus locations.

Because TAD has now been split into a development and a production environment (see the Improving the TAD Server Configuration for Reliability and Availability section), it has now become necessary to add the ability for the TAD Database Toolkit to be able to function in both environments. As such, a dropdown box was added to the Toolkit to enable the user to switch between the development and production servers.

Another important enhancement was the integration of the Trip Coherence algorithm (described in the Maintaining User Data Consistency After Transit Agency GTFS Data Updates section). A small checkbox was added for use when the user would like to perform this algorithm after updating the selected feed. These new features and several smaller user interface enhancements can be seen in the before and after screenshots of the TAD Database Toolkit application in Figure 19. 


\begin{tabular}{|c|c|}
\hline 㬰 & $\square \square$ \\
\hline \multicolumn{2}{|l|}{ Tools } \\
\hline \multicolumn{2}{|l|}{ Current Feeds } \\
\hline \multirow{6}{*}{$\begin{array}{l}\text { HARTLine Google Feed } \\
\text { BART Gov New } \\
\text { Metro St. Louis } \\
\text { PSTA } \\
\text { Miami-Dade }\end{array}$} & Use Selected \\
\hline & 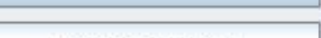 \\
\hline & Add New Feed \\
\hline & \multirow{2}{*}{ Modify Selected Feed } \\
\hline & \\
\hline & Exit \\
\hline
\end{tabular}

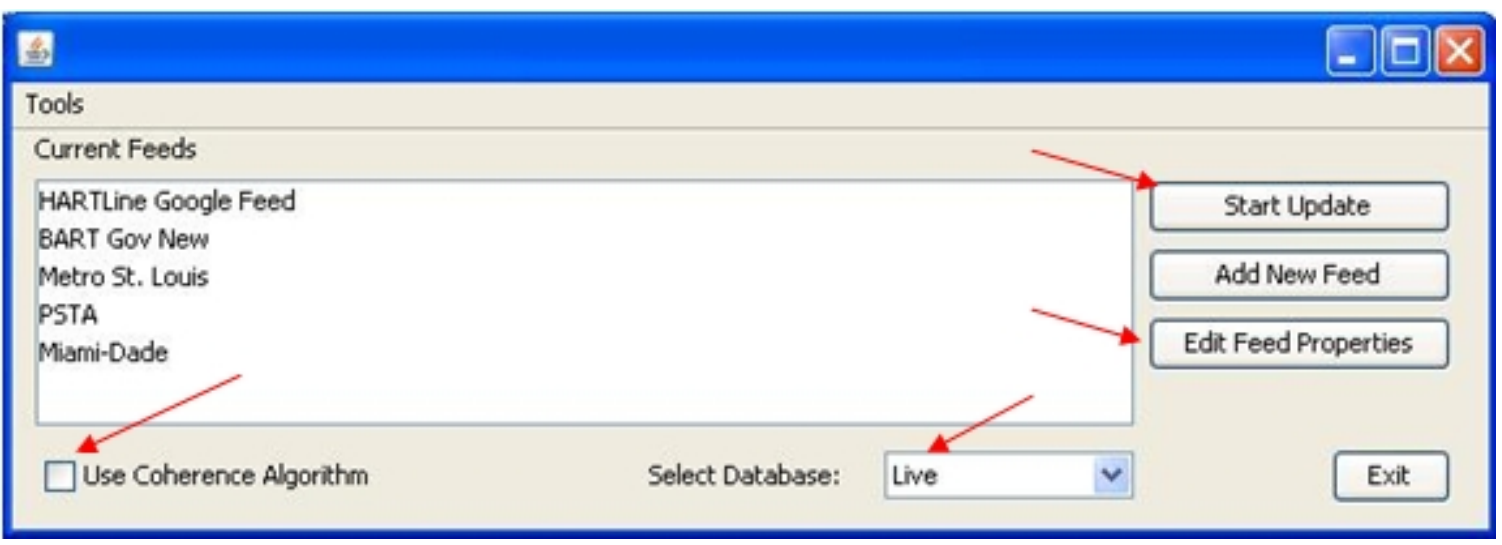

Figure 19 - TAD Database Toolkit, Highlighting the Interface Improvements and New Features 


\section{Chapter 4 - TAD Deployment Synopsis}

This chapter documents the level of effort required to deploy TAD at the selected transit agencies and results of deployment.

After selecting the agencies and making the necessary improvements to TAD, the research team arranged for phones with chargers to be delivered to the participating agencies. A welcome email was sent out to the agencies that included a user name and password for each tester for the mobile application and TAD Web site, a short how-to document for the mobile application and the Web application, a link to the TAD informational Web site, and a log sheet for recording test results and feedback.

\section{Miami-Dade Transit}

\section{Background}

Miami-Dade Transit (MDT) is the 14th largest public transit system in the U.S. and the largest transit agency in Florida (from MDT facts published at http://www.miamidade.gov/transit/about facts.asp). According to its homepage, MDT is the largest department of the Miami-Dade County government and is responsible for planning and providing all public transit services in the county. MDT prides itself on being an accessible service, offering mobility to people with disabilities. MDT's integrated transportation system consists of four major components: the Metrobus fleet, connecting most areas of Miami-Dade County; Metrorail, an electrically powered, elevated, rapid-transit system stretching 22.6 miles; Metromover, a 4.4-mile elevated people mover that serves the downtown central business district of Miami; and Special Transportation Service (STS), designed to meet the needs of people with disabilities who are unable to use regular transit services. Currently, MDT records over 326,000 daily (weekday) boardings on this unified system. STS's daily average is approximately 5,000 passengers.

Figure 20 shows MDT's informational Web site. The trip planning tool, located at the top right corner of the figure, links the rider to Google maps. 


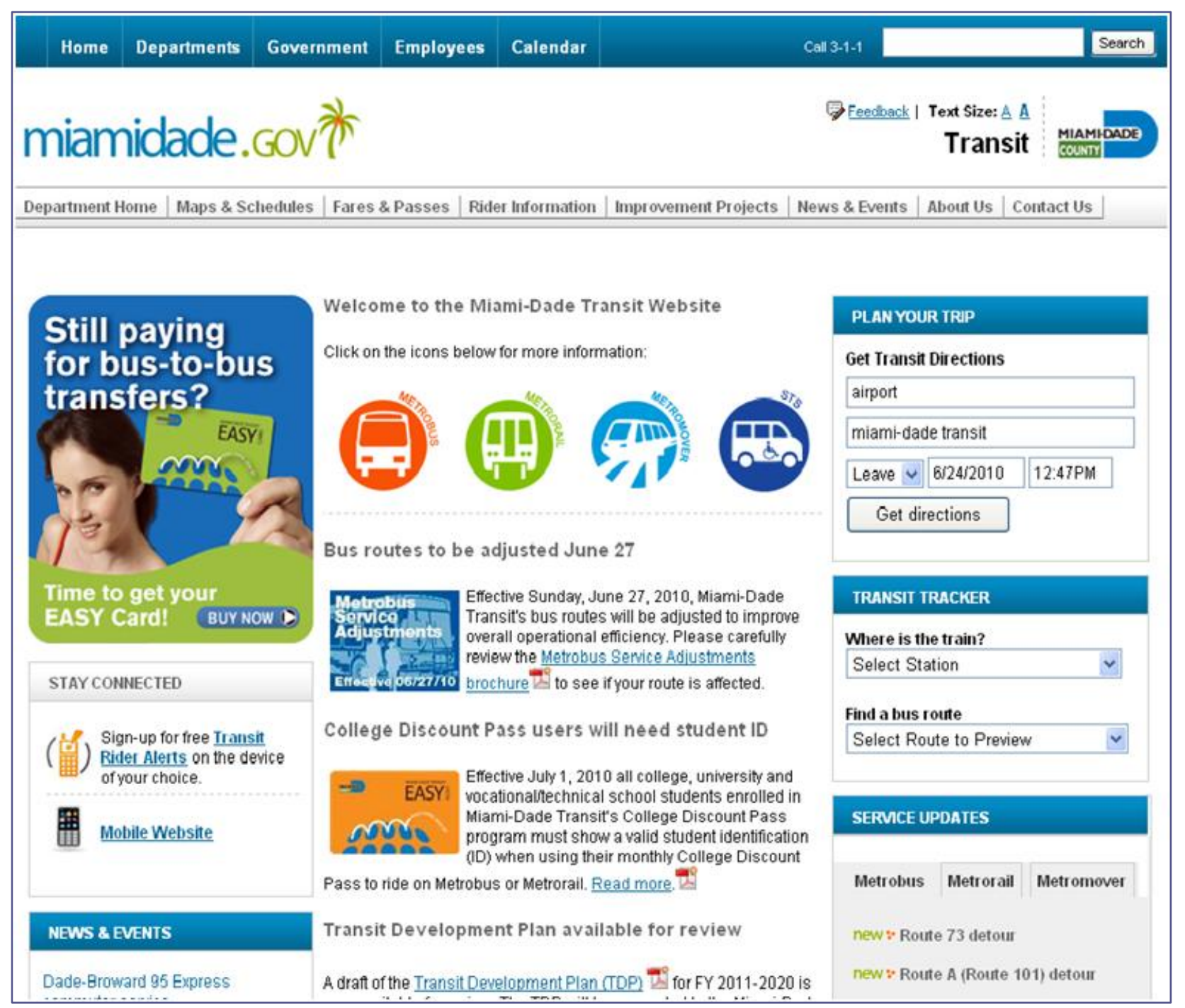

Figure 20 - Miami-Dade Transit Informational Web Site

\section{Logistical and Technical Issues}

Transit agencies often ask about the level of effort required to keep transit data up-to-date in the GTFS format for the Google Transit trip planner, as well as TAD and any other application that relies on the GTFS data. MDT provided the research team with an overview of the process required for each update of its GTFS data for each new markup period.

MDT's update process starts with an export of the agency's data from the Trapeze Export Tool for Google Transit Trip Planner, which is part of the scheduling software package provided by Trapeze. This tool requires Trapeze FX and one of Trapeze's Traveler Information Trip Planning Solutions (http://www.trapezegroup.com/solutions/pt google.php). After the Trapeze export, MDT runs the GTFS data through the Google Transit Feed Validator tool (http://code.google.com/p/googletransitdatafeed/wiki/FeedValidator). MDT must then 
examine the output of the validation tool and manually fix any errors that are identified in the GTFS data. For several reasons, these errors are not automatically fixed by the Trapeze GTFS export tool, and either the Trapeze data or the actual raw GTFS data must be manually adjusted in order for the validation tool to properly validate the data. A full description of this manual correction process is detailed in Appendix B. The validation tool also tends to generate many warnings, indicating that something might be wrong with the data. However, these warnings often can be safely ignored without impacting the Google Transit service. A description of some of these warnings can also be found in Appendix B.

MDT estimated that it takes one week from when the new data arrives from the scheduling department to when the updated GTFS file would be put on the MDT Web site for Google to retrieve.

The full process that Google requires for participation in Google Transit is documented at http://maps.google.com/help/maps/transit/partners/participate.html.

For TAD field testing, MDT did not need to make any modifications to their existing GTFS data feed. In other words, TAD was able to utilize the same GTFS data format that MDT has been providing to Google Transit for several years.

MDT originally requested and was given three phones for field tests, but ended up having only two testers within the agency available. As MDT does not employ a travel trainer, MDT delegated TAD testing responsibilities to one of their systems analysts and an engineering intern.

\section{Deployment Results}

Figure 21 shows the trips planned for MDT Tester One. MDT Tester Two also planned two trips for testing TAD. The third TAD user account and phone were not used by the MDT Testers.

The research team anticipated robust test results from MDT as a representative of the largest transit agency in Florida. It would have been interesting to observe how trips linked on buses, Metrorail, and Metromover. Since the testers of TAD at MDT were not trained to work with individuals with disabilities, these field tests were performed by MDT staff. The results of two test segments were reported by MDT, and the TAD worked successfully both times. Valuable feedback was provided from MDT's few short tests. MDT Tester One suggested that direction information be added to the trip planning section of the TAD Web site for situations where the agency does not provide headsign information in their GTFS data. One MDT Tester reported that he was unable to load a trip on the phone. After researching the issue, the research team discovered that the root cause of the problem was due to the trip having been accidentally duplicated during the trip planning process. This duplication may have occurred by double clicking or clicking twice on the Create trip button. The research team implemented a fix to prevent this issue from occurring again by disabling the button temporarily after clicking and displaying visual cues to the user that the server is processing the request. The MDT testers also provided helpful suggestions for improving the written instructions for using TAD. 


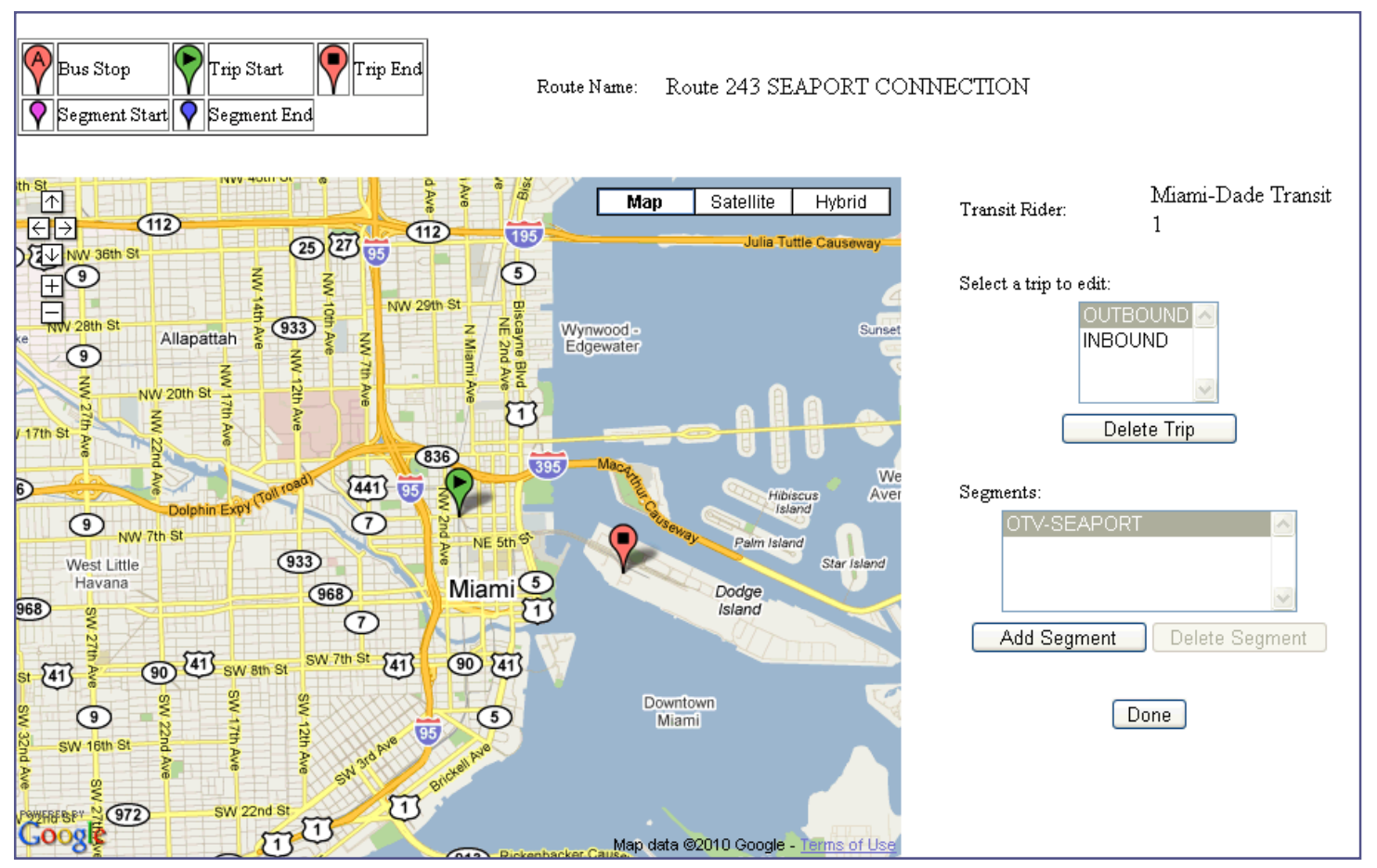

Figure 21 - MDT Tester One's Planned Trips

\section{Sarasota County Area Transit}

\section{Background}

In the last 30 years, the SCAT system has grown from six buses on nine routes to 44 routes, including 18 fuel-friendly hybrid buses on 29 routes. SCAT provides service to all of Sarasota County and parts of Manatee County. SCAT serves more than 2,500 bus stops. SCAT Plus provides paratransit service.

Figure 22 shows SCAT's informational Web site. SCAT posted an instructional video that helps riders use Google Maps to plan a trip. 


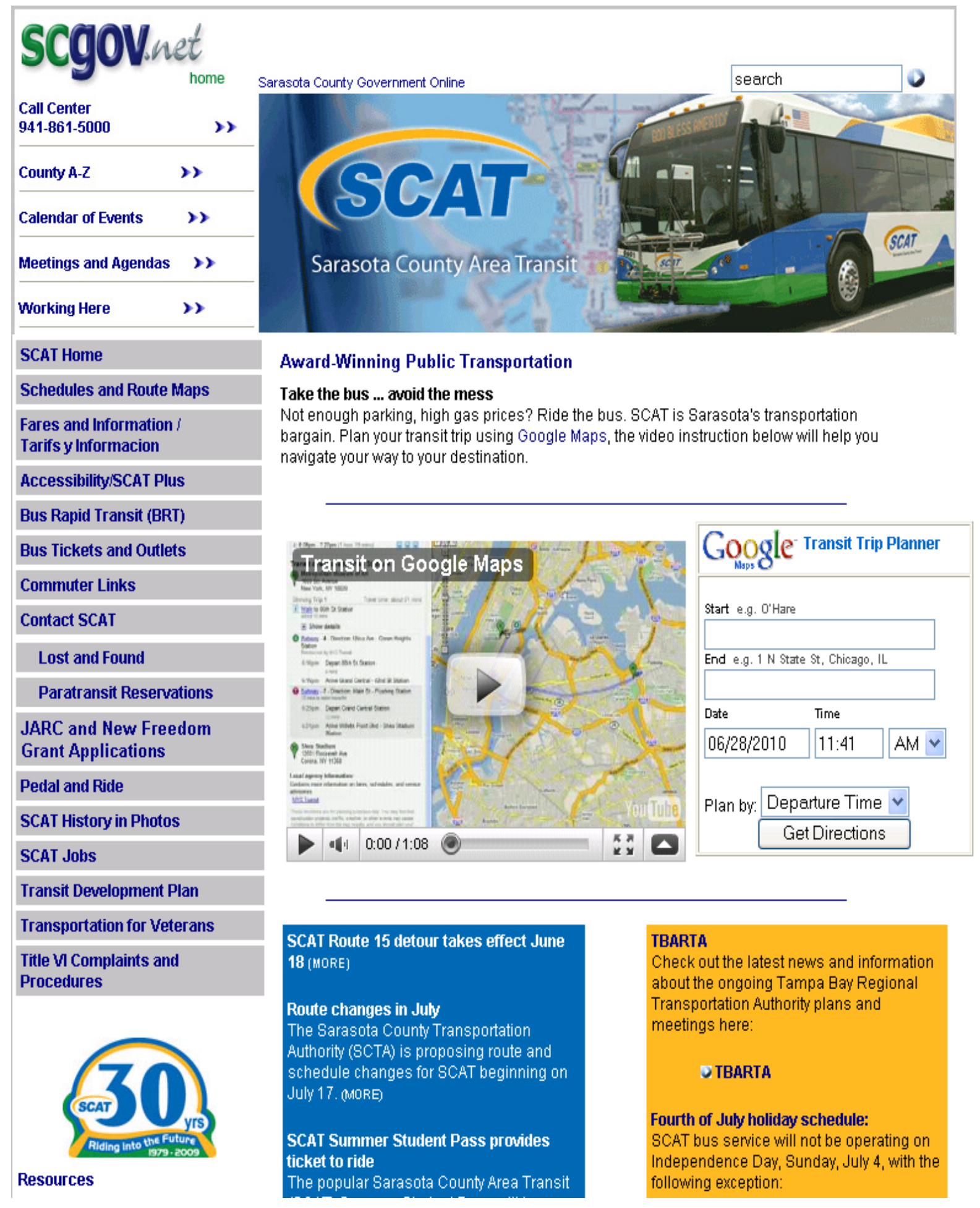

Figure 22 - Sarasota County Area Transit Informational Web Site 
SCAT's paratransit cost per passenger in 2007 was $\$ 27.92$ [14]. The fee for SCAT fixed-route service is $\$ 0.75$, with the discounted fare of $\$ 0.35$, with monthly service passes of $\$ 40$. By transitioning an able passenger who takes three roundtrips per week from paratransit to fixedroute service, the agency could produce a monthly saving of $\$ 630.08$. Since paratransit also often features limitations on the transit rider such as advanced 24-hour reservations and lengthy periods of waiting for pickup before and after a trip is taken, fixed-route public transportation can provide additional independence for able transit riders, including reduced cost to the transit rider. Additional cost savings and benefits related to travel training are documented in the report [14].

SCAT originally implemented their mobility training program to serve their customers during a paratransit service recertification period in 2003. During SCAT's paratransit recertification period, all paratransit customers were re-evaluated for qualification for paratransit service. SCAT provided mobility training to any customers who wanted assistance with fixed-route transit service, including those who did not qualify for paratransit after recertification. SCAT reports that since this initial period of activity, customer use of the mobility training program has declined. SCAT also offers financial incentives for paratransit applicants to use fixed-route service, including free fixed route bus passes to all passengers eligible for paratransit, and discounted bus passes at $\$ 2$ per month to individuals who do not qualify for paratransit.

While SCAT does currently offer mobility training services, its program seems to differ from HART and BCT's travel training programs. HART and BCT appear to have much higher demand for travel training, perhaps due to stronger connections with special education institutions that provide an ongoing influx of new travel trainees, especially individuals with disabilities and specifically young adults with cognitive impairments. For example, HART's travel trainer has reported being booked for more than two months in advance, indicating a very high-demand for travel training services. SCAT does not provide any formal documentation about their mobility training program and the program is not mentioned on the SCAT Web site. HART and BCT both provide information regarding their travel training program on their respective Web sites, including contact information for individuals interested in booking a travel training session.

SCAT contacted the research team in late 2009 after seeing presentation about TAD at the 2009 FPTA/FDOT/CUTR Professional Development Workshop and requested more information about the project. At the time, SCAT was preparing its data for the GTFS format for future use in the Google Transit trip planner, but did not yet have their GTFS feed in place. SCAT contacted the team again in early 2010 once they placed their data into the GTFS format and requested to be a partner in the TAD Deployment project. While as of July 7, 2010, SCAT is still not listed as a transit partner on Google's Web site, Google Transit has been available to plan trips in Sarasota since late May 2010.

TAD was able to use SCAT's GTFS data even before SCAT's GTFS data was published in the Google Transit service. The partnership with SCAT illustrates that an agency does not necessarily have to participate in Google Transit in order to leverage third-party applications which utilize the GTFS format. In other words, if a transit agency wants to expose their data to 
software developers so that more mobile and Web applications are made available to their customers, the agency can place their data in the GTFS format without having any relationship with Google or Google Transit. However, having Google Transit available, or another publically available automated trip planner, would help parents and guardians determine the routes and stops a rider should use to get from one location to another so that information can be entered into the TAD Web site.

SCAT did not make any modifications to their existing GTFS data feed for participation in the TAD Deployment project. In other words, TAD was able to utilize SCAT's exact GTFS data that SCAT had prepared for Google Transit.

\section{Logistical and Technical Issues}

SCAT Tester One reported difficulties with the trip planning process of the TAD Web application. The research team believes that this may stem from a lack of trip headsign information provided in SCAT's GTFS data. As a workaround, the list of trips shown on the Web site displays a list of trip IDs (Figure 23), which could be frustrating or confusing to the user since the number does not provide useful information to the transit rider. SCAT could enable TAD, and other applications which use SCAT's GTFS data, to provide more descriptive information to users by populating the trip_headsign field in the trips.txt file in their GTFS data with a text description that describes a route to a passenger (e.g., To Downtown, To Airport). Typically, this head sign text that is shown on the bus head sign that riders can see as a bus is approaching. TAD could also construct text that could substitute for headsign information based on the description of the last stop of the line (e.g. Direction Venice Train Depot), similar to Google Transit. However, this text may not match the actual headsign of the bus, so the direct population of the headsign field by the transit agency is preferred.

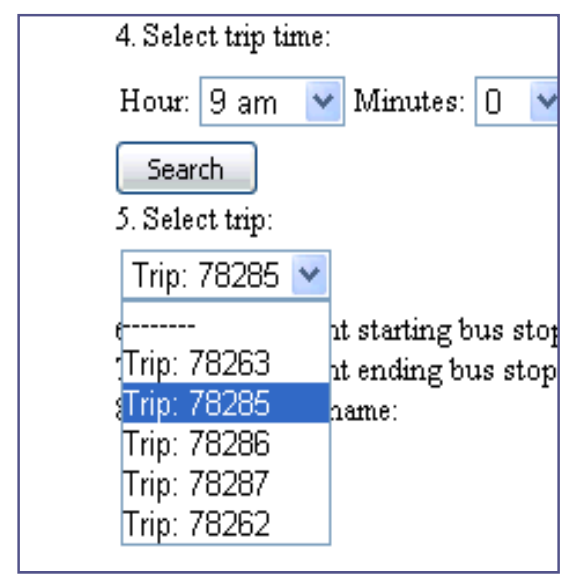

Figure 23 - Trips Listed by ID when Searching for Trips from SCAT Routes 


\section{Deployment Results}

Figure 24 displays an example of a trip planned by SCAT Tester One, including a transfer point from one route to another. SCAT used TAD for three trip segments, and the TAD performed successfully in each test.

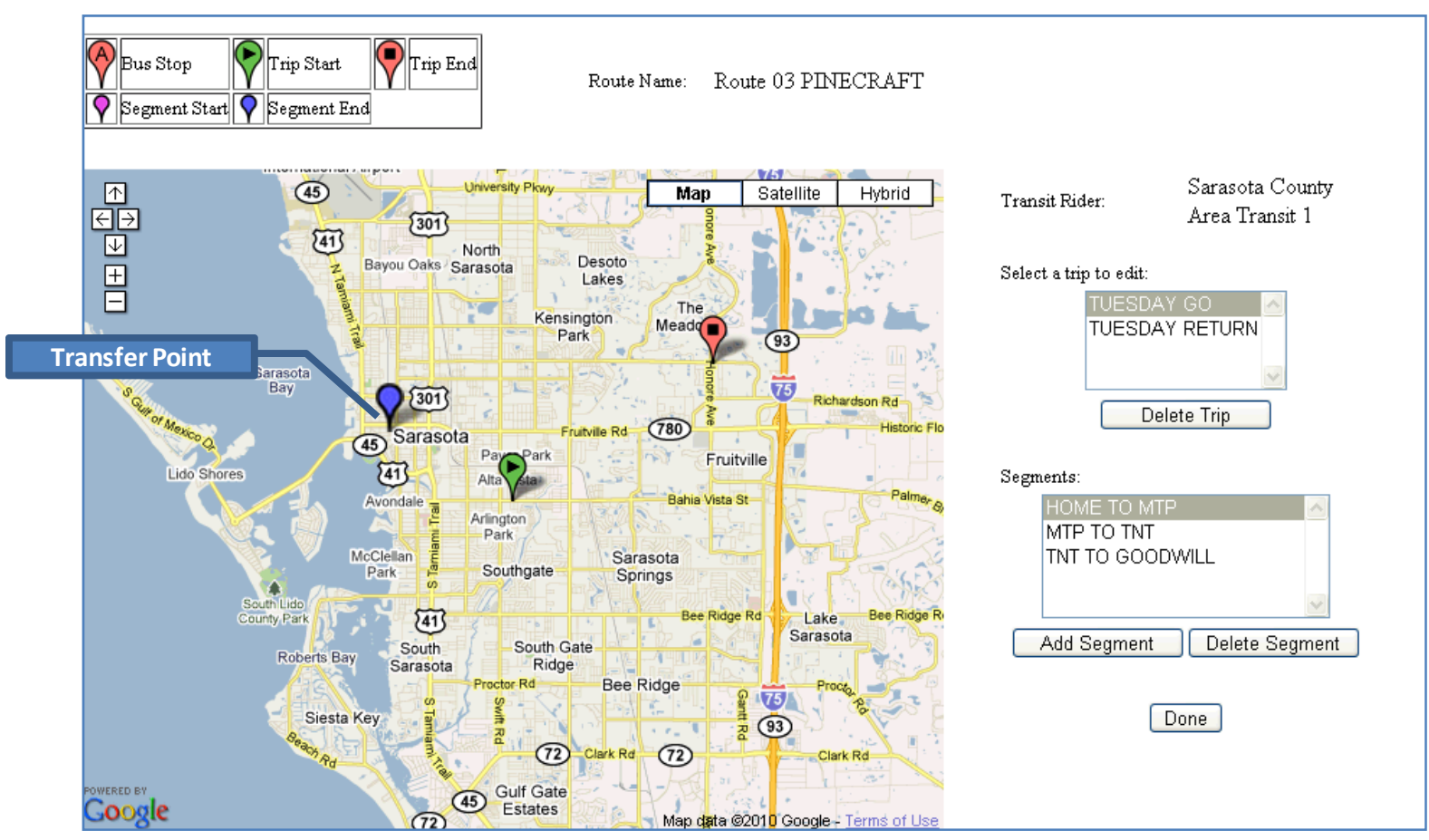

Figure 24 - SCAT Tester One's Planned Trips

SCAT's mobility trainer expressed some concern over the ability of a transit rider with cognitive impairments to plan their own trips via the TAD Web site. This is a valid concern, since planning a trip via the Web site involves complex time and logic concepts that may not be possible for some trainees with cognitive impairments. Another concern expressed by the SCAT mobility trainer was that if TAD was released to the general public and SCAT was required to plan trips for all users, this process would be too time-consuming and take away from other normal work activities and responsibilities.

Since the same complex time and logic concepts required for TAD Web site trip planning are also required to successfully navigate the transit system, the research team anticipates that most transit riders with cognitive impairments who may not be able to plan trips to unfamiliar locations using the TAD Web site will also require travel training for trips to any new location. Therefore, the research team expects that TAD will be a tool used by travel trainers and trainees with cognitive disabilities as part of the travel training process. TAD has never been intended to be a replacement for travel training. As previously mentioned, it takes 23 skills to complete a transit trip including walking to the correct bus stop, knowing which route to take, recognizing the correct bus to board, paying fare or using pass, etc. 
Travel training teaches these skills to an individual with a focus on a specific route. New routes require re-training on recognizing the bus number, where to board, recognizing a landmark to request a stop, and exiting at the correct destination. For many individuals, TAD could be a safety net that is left with the person after the travel trainer moves to their next trainee that helps them with one of the most difficult skills, knowing when to get off the bus. Parents, friends, or family members could also assist in the TAD Web site trip planning process. HART's travel trainer noted that he believes TAD could help expedite the training process for some clientele, which could actually give the trainer the ability to train more clients in the same amount of time. To assist travel trainers in planning trips for trainees, automated trip planning capabilities could be integrated into future versions of the TAD Web site.

\section{Pinellas Suncoast Transit Authority}

\section{Background}

The following are selected PSTA facts and figures from PSTA's Web site at

http://www.psta.net/.

$\square$ Transit vehicles - 205

$\square$ Routes - 38, including 2 express routes to Tampa

$\square$ Bus stops - 5,368

$\square$ Shelters - 712

$\square$ Customer service centers - 4

$\square$ Annual calls received by InfoLine - 330,771

口 Total ridership - 12.2 million annually; 43,528 average weekday

$\square$ Bikes on buses trips - 353,262 annually; 1,300 average weekday

- Paratransit trips (American With Disabilities Act) - 228,463 annually; 747 average weekday

$\square$ Number of employees - 562

The Google Transit trip planner link is prominently displayed in PSTA's informational Web site, as shown in Figure 25. 


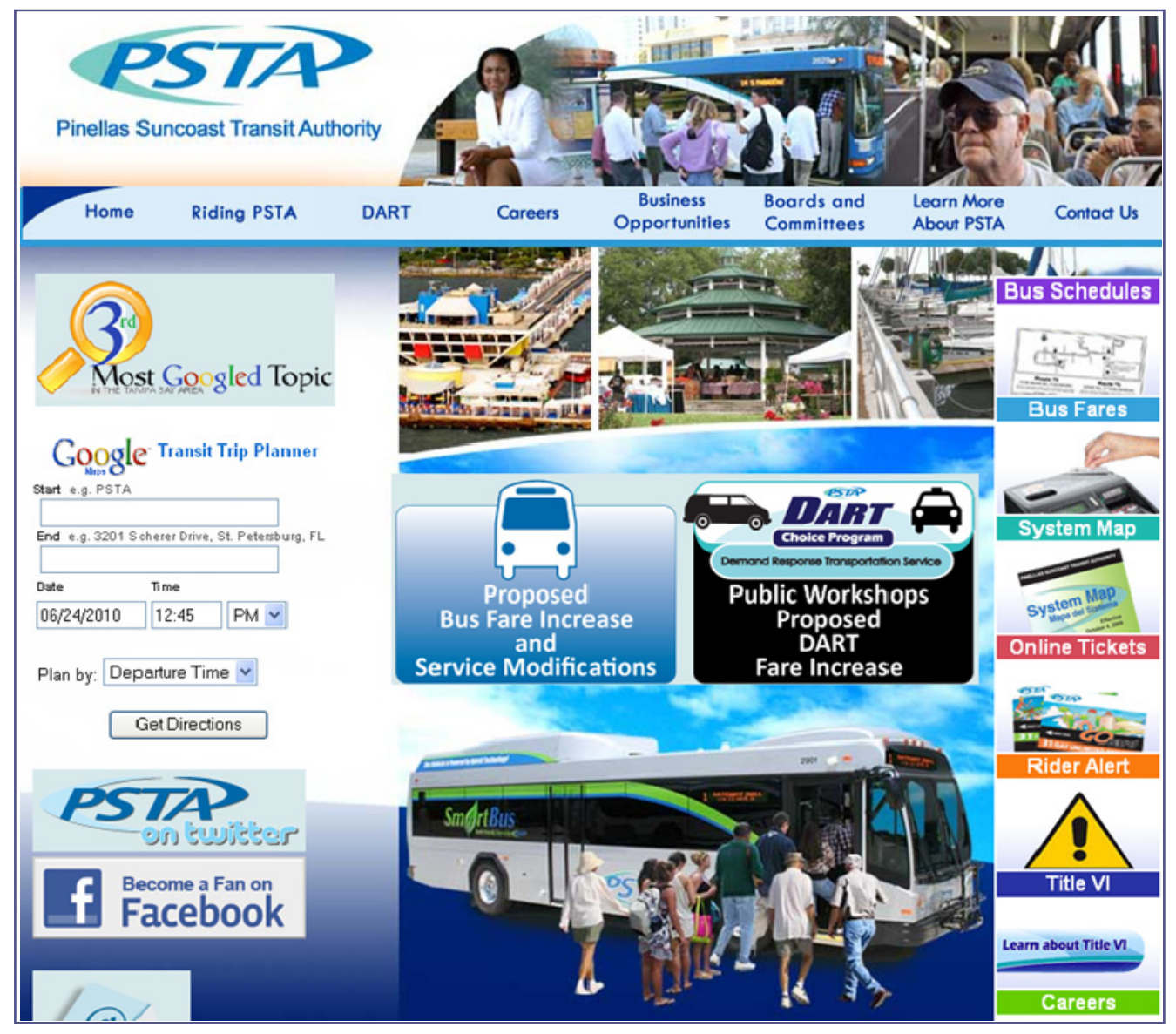

Figure 25 - Pinellas Suncoast Transit Authority Informational Web Site

\section{Logistical and Technical Issues}

Upon attempting the first field test with TAD, PSTA Tester One alerted the research team that she was unable to correctly view her trip on the phone. In an email, she described that the trip information was not showing on the phone (see Figure 26). After PSTA Tester Two reported the same difficulty, it became clear that there was an issue with the TAD mobile application that occurred only with PSTA trip data.

The research team planned trips with PSTA routes and was able to replicate, debug, and resolve the issue. Eventually, the team determined that the cause of the issue stemmed from PSTA's GTFS data having the bus stop ID's in an alphanumeric format which prevented the proper trip information from displaying on the phone. All other agencies that had previously tested TAD had numeric values for the bus stop ID, so the TAD system expected numeric bus stops IDs for PSTA as well. Once the team was able to reproduce the issue, they sent the PSTA testers a picture of the phone's screen taken with a digital camera (see Figure 26) to confirm that the issue was the actual issue the PSTA testers encountered. PSTA confirmed the issue, and the research team proceeded to modify the TAD mobile application as well as the TAD Web service to accept alpha-numeric values for bus stop IDs. These significant changes within TAD meant 
that all TAD testers across all participating agencies would need to update the TAD mobile application to a new version in order to continue testing. The research team provided instructions to each agency and posted an instructional video demonstrating how to update the mobile software on the phone via the Over the Air (OTA) update. Each agency successfully updated their mobile applications on their phones, using these instructions, by clicking on a link in a text message to trigger the update.

No changes to PSTA GTFS feed was required for TAD to import the data into the TAD system.

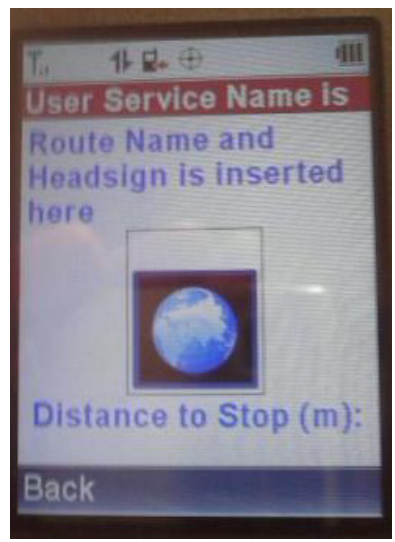

Figure 26 - Screen Shot of TAD Cell Phone Application with Blank Trip Screen Due to Technical Issue with PSTA's GTFS Bus Stop Data

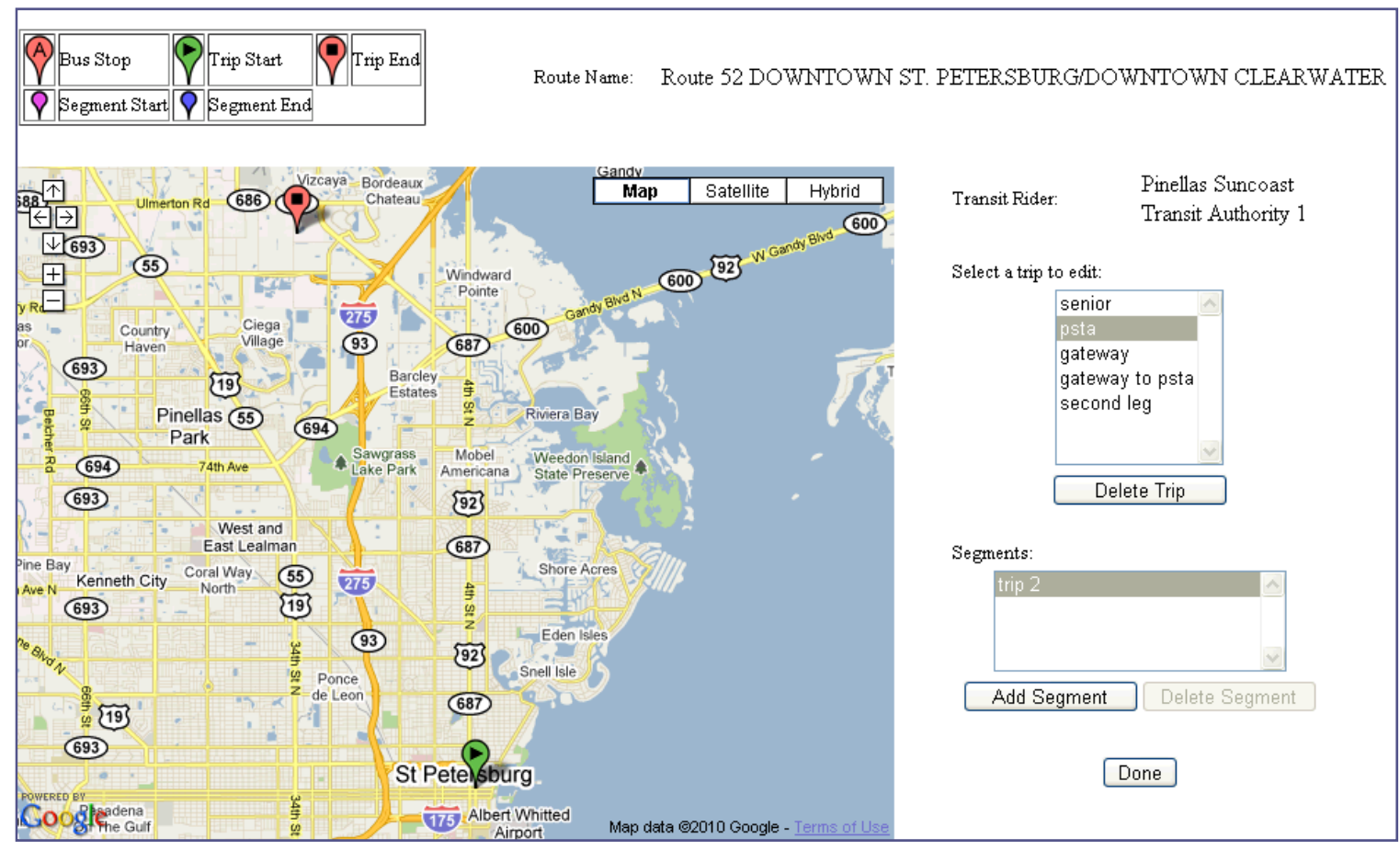

Figure 27 - PSTA Tester One's Planned Trips 


\section{Deployment Results}

After the alphanumeric bus stop ID issue was resolved, TAD testing was carried out on 11 trip segments by PSTA (Table 2). Figure 27 shows a sample trip planned by a PSTA Tester. Eight of the 11 Pull the Cord Now prompts were successfully given by TAD in an ideal location for the transit rider to comfortably request a stop in time to exit the vehicle at their destination stop. The occasions where TAD gave either late or no prompts are discussed below.

Table 2 - Field Test Results from PSTA

\begin{tabular}{|l|c|}
\hline Ideal Prompts & 8 \\
\hline Early Prompts & - \\
\hline Late Prompts & 1 \\
\hline No Prompt Given & 2 \\
\hline Total Number of Trip Segments & $\mathbf{1 1}$ \\
\hline
\end{tabular}

On May 26, PSTA Tester One logged that she had taken a trip and received the Get Ready prompt but did not hear the Pull the Cord Now! prompts until after she exited the bus. Figure 28 shows the GPS trail from the trip she took using TAD. The green markers indicate the starting bus stop (Start), ending bus stop (End), and the second to last (STL) bus stop. The red markers show where TAD issued the Get Ready prompt and the Pull the Cord Now! prompt were given. 


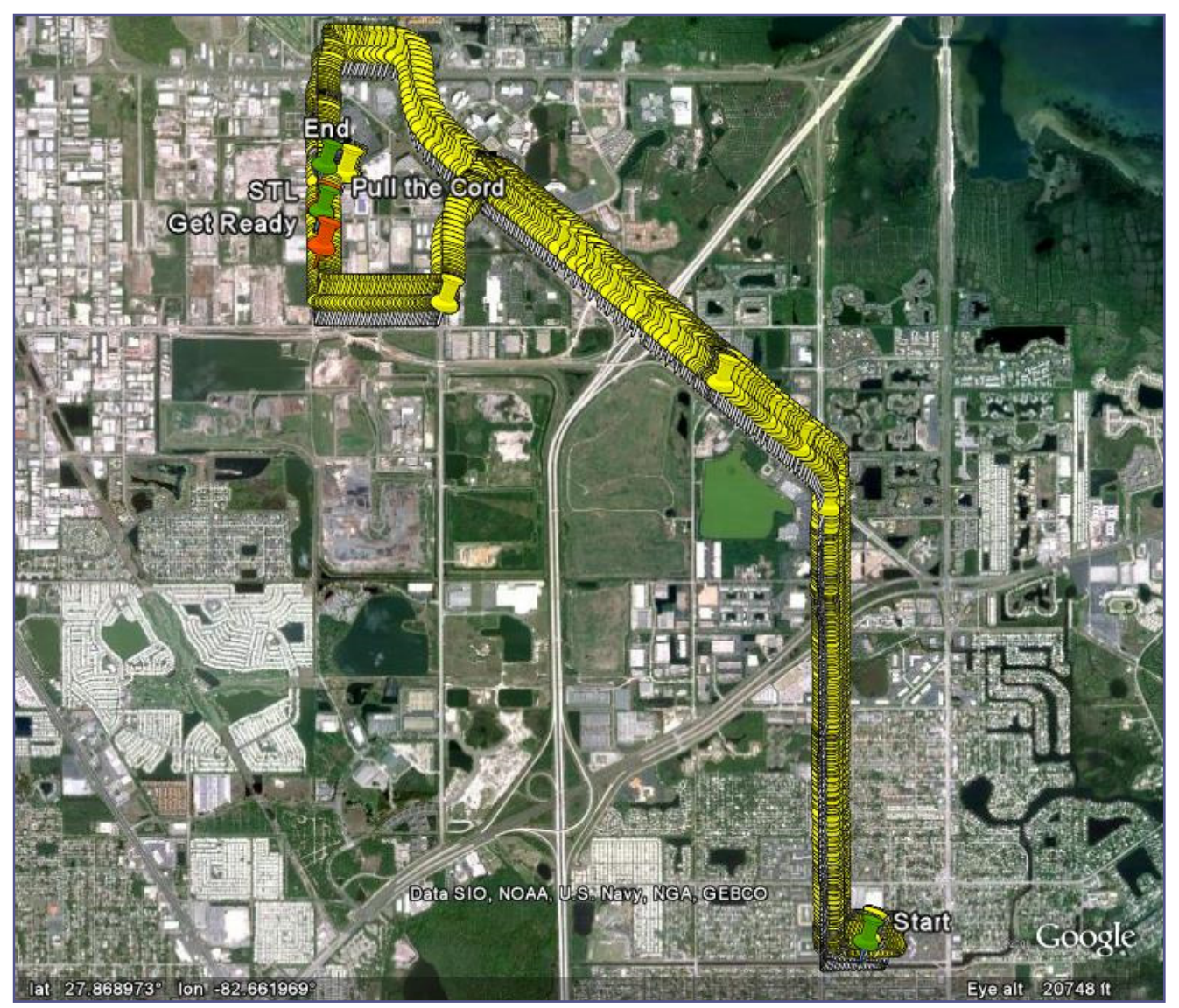

Figure 28 - PSTA Tester One's Trip on 5/26/10

Zooming into the map, in Figure 29, shows that the Pull the Cord Now! prompt was issued by the phone near the second to last bus stop, which was before the ending stop. Therefore, the data from the system indicates that the alert was delivered in the correct location, while the observer using TAD reported a late alert for this trip. One possible explanation for this discrepancy is that that the phone's CPU was under a high processing load at the moment when the TAD software triggered the alert, but the user did not see the alert until a few seconds later when the CPU actually processed the alert request from the TAD mobile application. This CPU overload and processing delay could be due to the current testing mode of TAD, which immediately sends each GPS fix back to the TAD server for later analysis. To reduce this processing load on the CPU, the TAD application may need to send fewer fixes to the database for storage, or GPS fixes need to be queued into a larger package of fixes that are occasionally transmitted in bulk to the server. The queuing solution is preferred; since transmitting fewer fixes to the server means that there is less information to troubleshoot future TAD problems. 


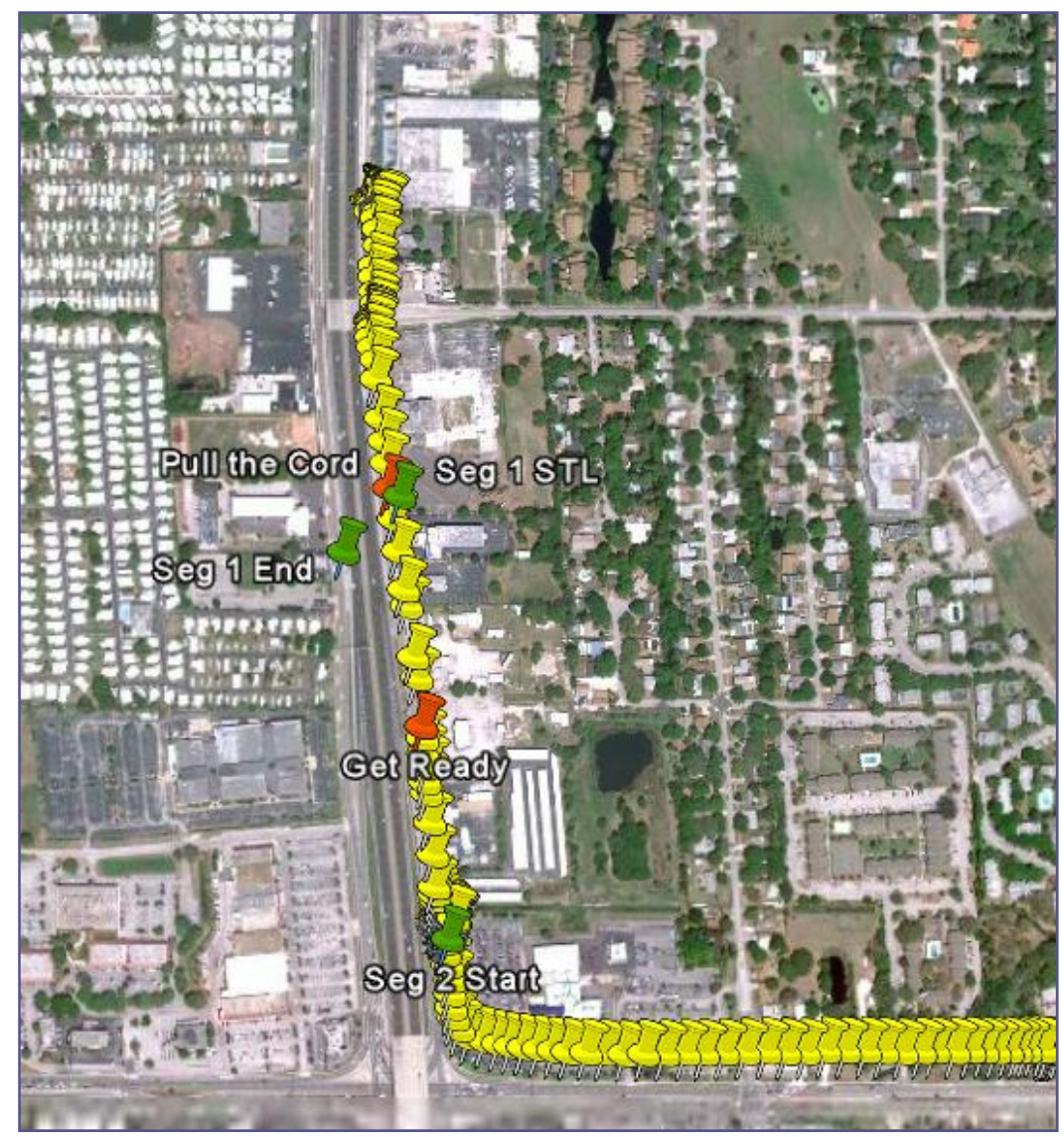

Figure 29 - End of First Segment of Trip and Beginning of Second Segment where Tracking is Temporarily Non-Functioning

Figure 30 presents a closer look at where the prompts were issued by TAD in relation to the second to last (STL) and ending (End) bus stops. This issue was not reported in any other tests, so, if CPU processing load was the issue that resulted in the delayed alert, this circumstance only happens occasionally. 


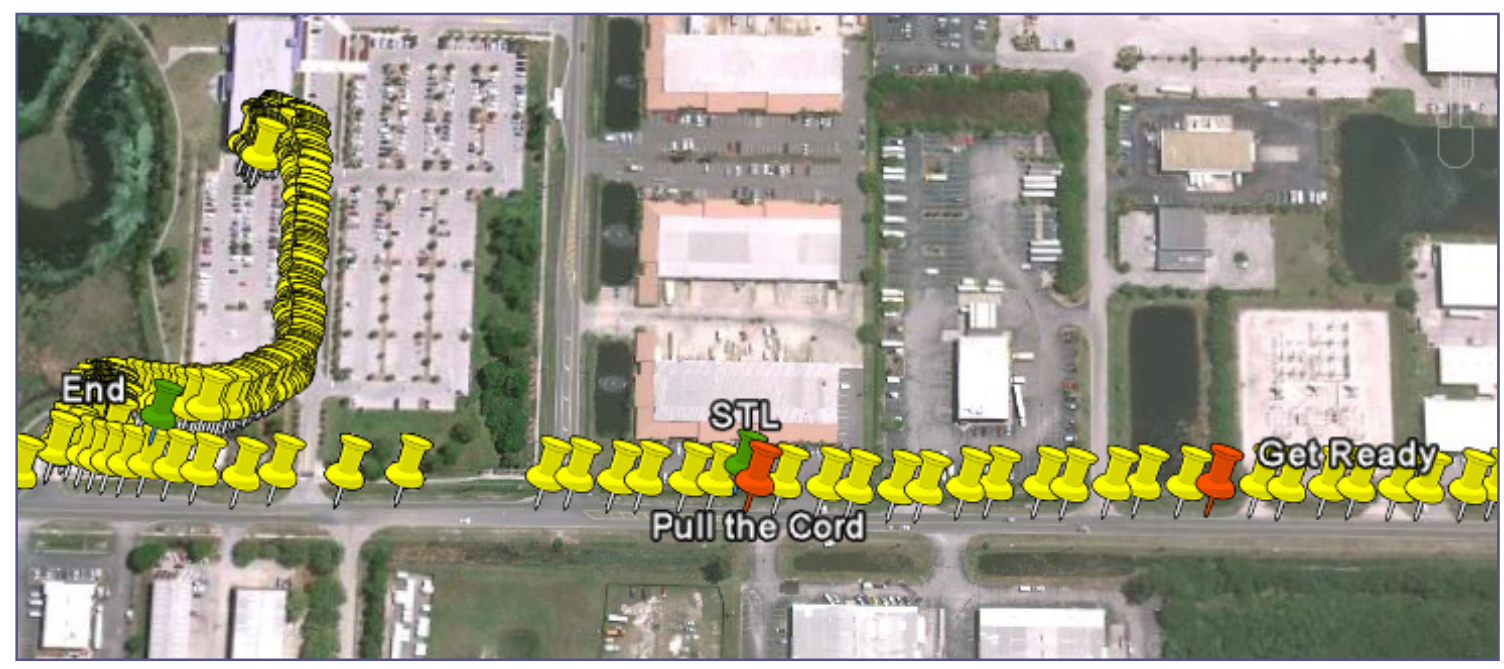

Figure 30 - TAD Prompts in Relation to the Second to Last (STL) and Ending (End) Bus Stops

PSTA Tester Three reported that he did not receive the Pull the Cord Now! prompt for two of the three segments in a trip he took. In the log sheet, he reports that the first segment worked well but that he did not receive the alerts for the second segment and did not realize it until it was too late. After he completed the third segment of his trip, he noticed that he had forgotten to manually activate the skip segment feature on the phone so that TAD could attempt to give the prompts correctly for the third segment even though it had not given the prompts for the second segment. Figure 31 shows the tester's trip where nearly the entire second segment of the trip was not recorded in the TAD database. These missing tracking points could be due to a number of reasons including lack of data connection, frozen phone, TAD mobile application, Web service error, database issues, or user accidentally canceling the trip or closing the TAD application. Figure 31 shows that TAD gave the Get Ready and Pull the Cord Now! alerts for the first segment shortly before the tracking points ceased to be recorded, which caused the alerts for second segment to be missed. Further testing and analysis will have to take place to determine why no data was reported for the second segment of the trip. 


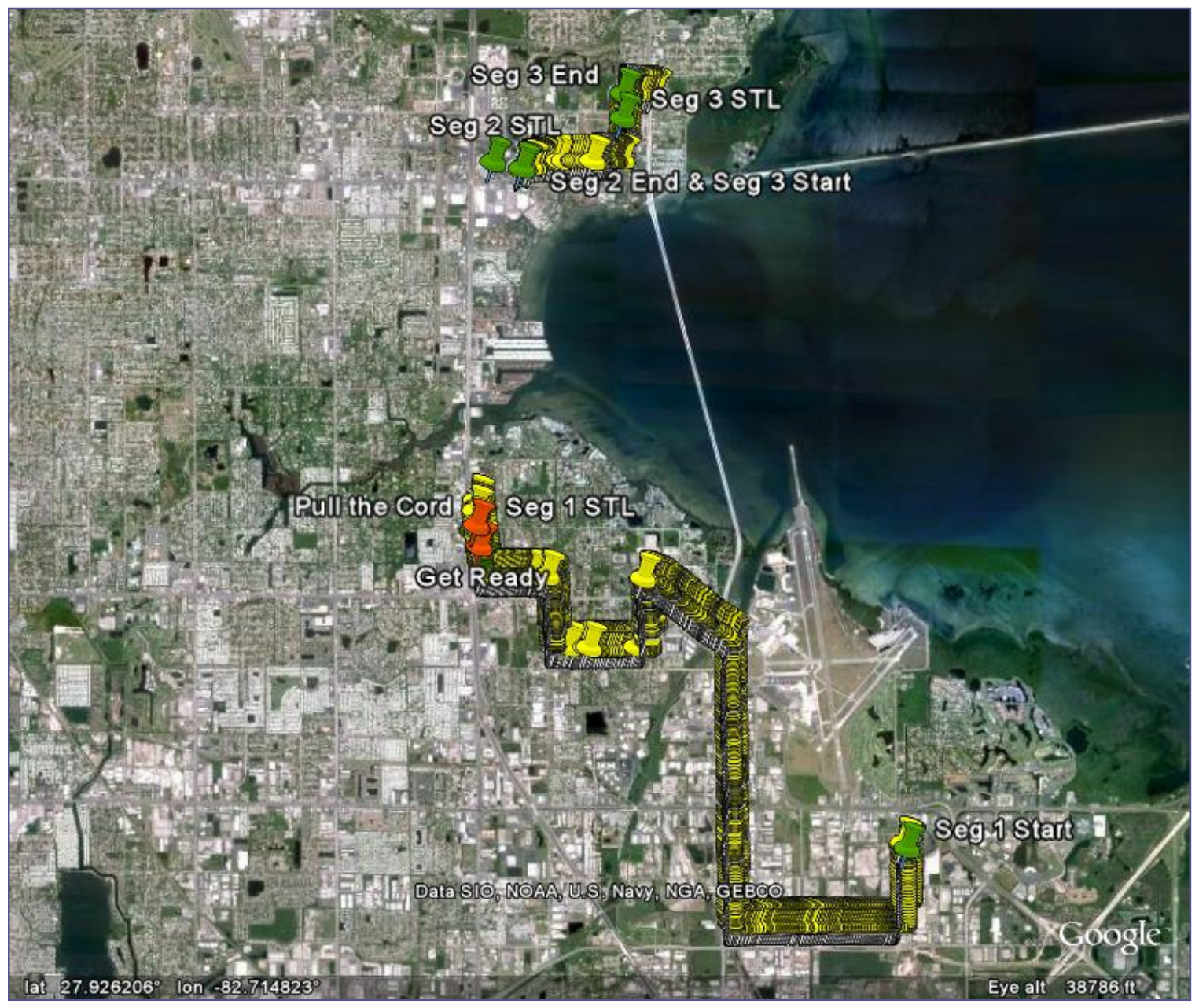

Figure 31 - PSTA Tester Three's Trip from 6/3/10 shows a gap in GPS data

\section{Broward County Transit}

\section{Background}

The following are selected BCT facts and figures from BCT's Web site, www.broward.org.

$\square$ Service area - 410 square miles within Broward County; BCT buses connect to Palm Beach and Miami-Dade Transit systems and to Tri-Rail

$\square$ Total active fleet - 303 fixed-route buses and 70 community buses

- Paratransit service routes - 41 on weekdays, 37 on Saturdays, 35 on Sundays

$\square$ Designated bus stops - 4,827

$\square$ Bus shelters - 509

$\square$ Annual service mileage - 13.9 million miles

$\square$ Passengers - 120,620 daily, 36.8 million trips annually

$\square$ Telephone requests - 1.3 million annually 
The online trip planning via Google Maps can be seen in a small pane on BCT's informational Web site, as shown in Figure 32.

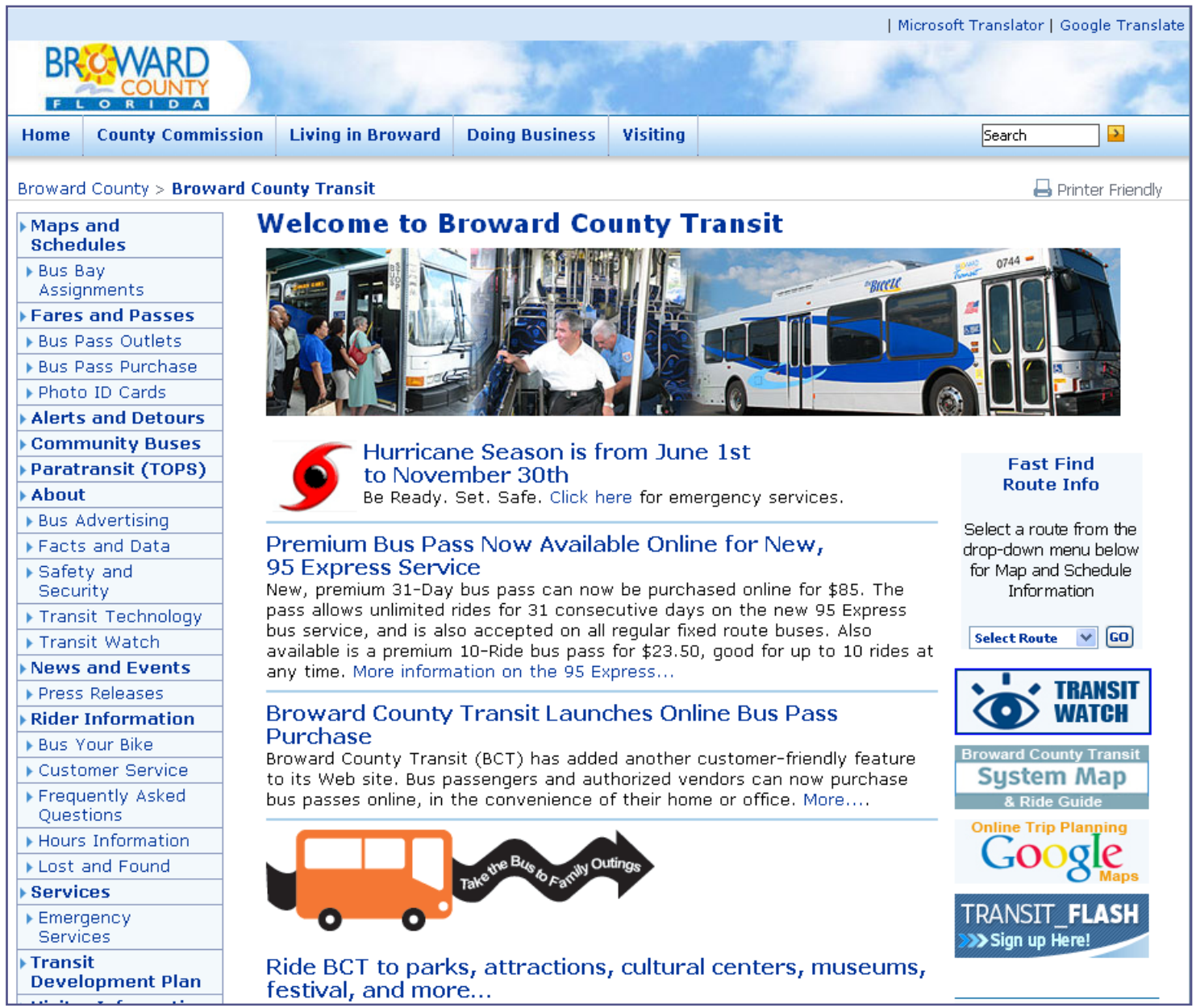

Figure 32 - Broward County Transit Informational Web Site

\section{Logistical and Technical Issues}

BCT has a company policy of blocking internet communication on certain ports, which included the port on which the TAD Web application runs. The BCT tester reported this problem the first time she attempted to plan a trip using the TAD Web site. She submitted a request to the appropriate BCT group and requested approval from two higher levels, which was eventually granted to provide access to the TAD Web site.

BCT's GTFS dataset was minimal in comparison to the other transit agencies, and it appears that the agency is still in the early stages of populating their GTFS data feed. For all GTFS datasets observed prior to BCT, if a transit agency did not have information for a particular field in the GTFS data, it was left blank. In the case of BCT's GTFS headsign information, the headsign field was omitted entirely, which required a few minor code changes within the TAD system. As 
mentioned earlier, Google Transit estimates the routes headsign using the last stop on the line (e.g. Broward Central Terminal). However, it is preferred that the agency populates this headsign field if possible, since the last stop of the line may not match the actual headsign shown on the bus.

TAD was able to import and use BCT's GTFS data without any changes to the GTFS data on the part of BCT.

\section{Deployment Results}

Figure 33 shows a trip planned by BCT Tester One on the TAD Web site. The trip contained two segments and TAD successfully gave both alerts at the appropriate times. Due to the temporary inability of the BCT Tester to access the TAD Web application and other small roadblocks, BCT Tester One was only able to test one trip with TAD.

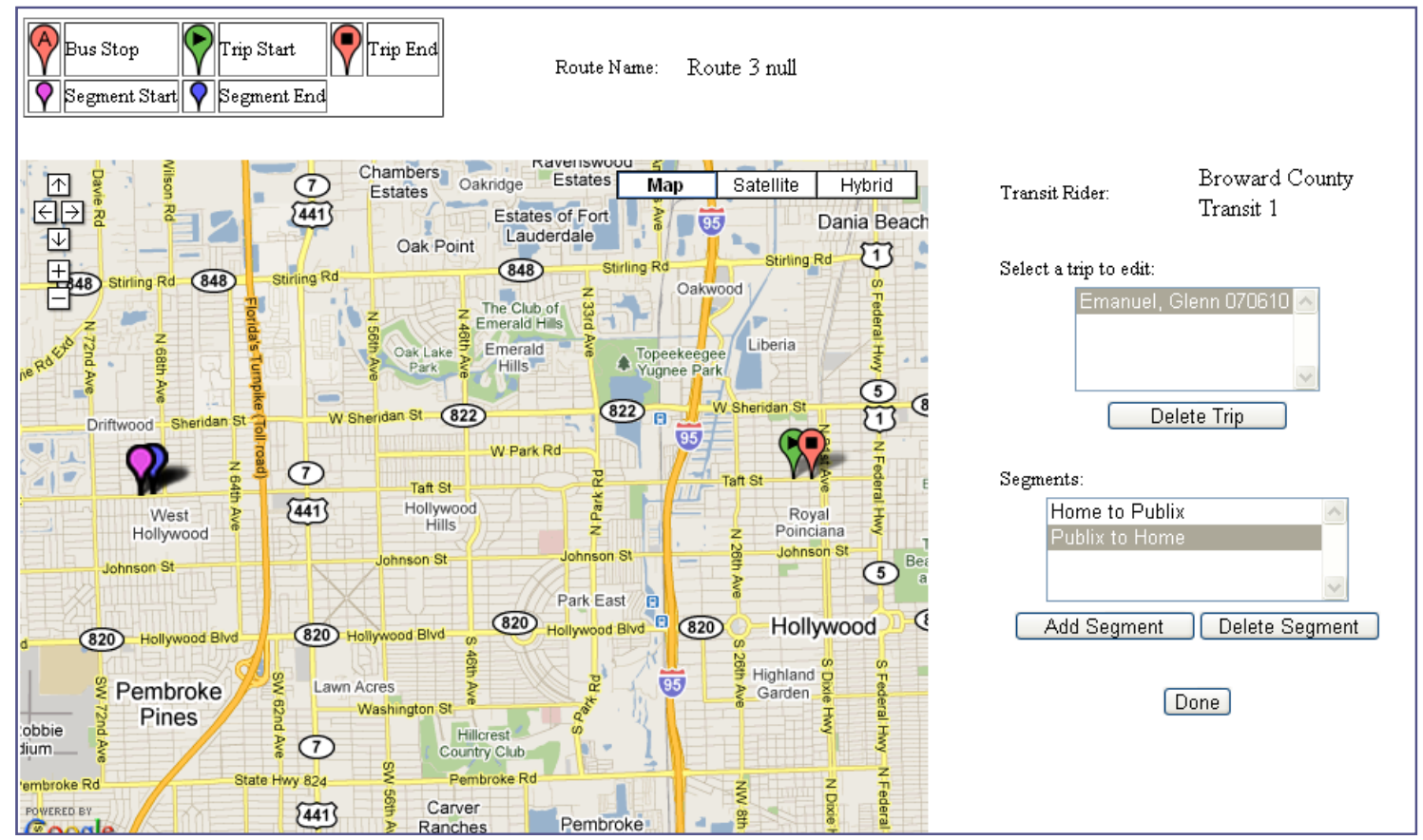

Figure 33 - BCT Tester One's Planned Trips

BCT Tester One gave some good feedback on the TAD system, including a request for the Web site to have the ability to perform automated trip planning. Currently, TAD users must use other tools such as Google Transit to get a series of routes and bus stops to travel from simple start and destination locations. During the development of the TAD Web site, travel trainers requested fine-grained control over the exact stops where the user would board and exit the vehicle so that they could take into consideration personal limitations (e.g., the inability to safely cross the street) when planning trips for their trainees. However, there are situations where an automated trip planning may be able to provide adequate directions for a trainee, which could then be edited by the travel trainer for fine details. Future versions of the TAD Web site should 
consider the implementation of automated trip planning, while allowing travel trainers to edit planned routes for individual considerations.

BCT Tester One also noted that she believes that TAD will be useful to individuals with visual impairments as well as individuals with cognitive impairments.

\section{Discussion of Results of TAD Deployment}

Table 3 summarizes the results of field testing as reported to the research team by all the participating agencies.

Table 3 - Summary of Field Test Results from All Participating Transit Agencies

\begin{tabular}{|l|c|c|c|c|c|}
\hline & $\begin{array}{c}\text { Ideal } \\
\text { Prompts }\end{array}$ & $\begin{array}{c}\text { Early } \\
\text { Prompts }\end{array}$ & Late Prompts & $\begin{array}{c}\text { No Prompt } \\
\text { Given }\end{array}$ & $\begin{array}{c}\text { Total Number of } \\
\text { Trip Segments }\end{array}$ \\
\hline MDT & 2 & & & & 2 \\
\hline PSTA & 8 & & 1 & 2 & 11 \\
\hline SCAT & 3 & & & & 3 \\
\hline BCT & 2 & & & & 2 \\
\hline All & 15 & & 1 & 2 & 18 \\
\hline
\end{tabular}

Overall, TAD performed very well in each of the four new cities tested under this project, with 15 of 18 prompts to cell phone users being given in an ideal location. These results are from first set of TAD tests run in each city by each local agency, without any testing in advance by the research team. Therefore, to improve performance, it is expected that the research team can further investigate the three instances where prompts did not appear in the ideal location.

The research team made a number of observations during the field tests with the transit agencies that will aid in the further improvement of the TAD system. For example, to allow users at the transit agencies to use the bus stop modification functions on the TAD Web site, all users were assigned to an administrator role. The administrator role grants the user functionality far beyond the bus stop modification section and, since there was no personalized training provided, some of the users were not well prepared to use some of the more advanced features of the TAD Web site. Many mistakes in using the Web site seemed to stem from the fact that under the administration role, all users (including other transit riders) are shown in the list on the main Web site page. Since the testers were performing the role of both the travel trainer and the transit rider, some users forgot to pick their user as the selected transit rider before clicking the Create a new trip button on the TAD Web site. In other words, the users assumed that the new trip being created would automatically be assigned to their own transit rider profile. However, if a user has an administrator role, they are permitted to create trips for any user in the system. This caused several new trips to be accidently created for a different user who happened to be the first transit rider in the list. It also created confusion because, later, the user would be unable to find the trips he/she created under their profile and would think something was wrong with the TAD system. 
To alleviate this issue, two approaches can be taken to enhance the usability of TAD. First, access to the bus stop modification tool should not require administrator privileges. Instead, a new feature could be added to TAD that assigns a travel trainer to a specific transit agency. Once the travel trainer is assigned to the agency, they would be allowed to modify bus stops only for that agency. This feature would show only the bus stop editor to travel trainers, without showing all the other advanced administrative features. The second approach is much simpler; require the user to physically click on a transit rider's name before clicking the Create a new trip button instead of automatically using the first user in the list. If only one transit rider is listed, this new requirement is waived.

One of the biggest challenges the test users faced was figuring out how the TAD system works without being oriented with the system by an experienced TAD user. The research team worked to prepare documentation to help guide the new TAD testers through the process of planning and conducting trips. Screenshots of the Web site and the phone emulator were provided for visual reference on the informational Web site. As new issues arose and were fixed during the course of testing with the travel agencies, a new version of the TAD mobile application was released and the testers were requested to upgrade the mobile application on their phones. Due to limitations of the phone emulator, the research team was not able to provide screenshots or a screencast of the TAD mobile application upgrade process from the phone emulator software. Instead, the process was filmed with a team member demonstrating the steps on an actual phone. The video was posted on the TAD informational Web site along with written instructions to make it easier for the testers to follow along and perform the upgrade process. After the instructional video was posted to the Web site, no users seemed to have any problems updating to the new version of the mobile phone application.

Another difficulty faced was in communicating issues with the TAD mobile application. Testers from PSTA discovered an issue in which when they tried to test a trip on the phone, but the trip information was not correctly populated on the phone's screen. Since the testers were not able to take a screenshot, they needed to remember what they saw so they could later send the research team a written description of the screen. After replicating the behavior described by the testers, the research team took a digital photo of the phone screen and emailed it to the testers for confirmation that the behavior was indeed the issue they had encountered.

This project is the first attempt to have users in remote locations log into the TAD Web site on their own without face-to-face training, so it is a good chance to gather user feedback that can be used to improve the learning-curve of the Web site. Many testers commented on the Create a new trip section of the TAD Web site. This is, of course, the main function of the TAD Web site, and it seemed to create the most frustration. Although the testers were expected to be familiar with their transit agency's routes and bus stops, this may not have always been the case, especially for testers who were not travel trainers and did not travel the transit system as part of their everyday job. Some testers gave feedback that they had difficulty identifying the stops or sometimes selected the wrong stop. One tester said that every time she selected a wrong stop, she would press the back button in her Internet browser, log in to TAD again, and begin the trip inputting process again. The research team added several new enhancements to this section of 
the TAD Web site in order to address the most frequent comments. Functionality was added to display bus stop names upon mousing over the bus stop (Figure 34), and a button was added to clear the currently=selected bus stops (see Figure 35). Other planned enhancements for increasing the ease of use of trip planning in TAD include a button to remove a segment and renaming the Done button to a clearer phrase such as Return or Finished. Additionally, the page could be adapted to handle clicks on the back button of the Internet browser in order to prevent the user from accidentally terminating their TAD session.

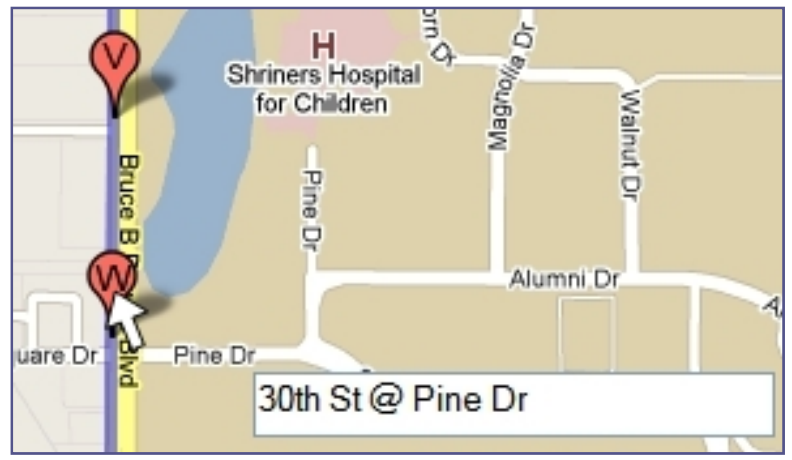

Figure 34 - New Bus Stop Identification Feature on Mouse-Over

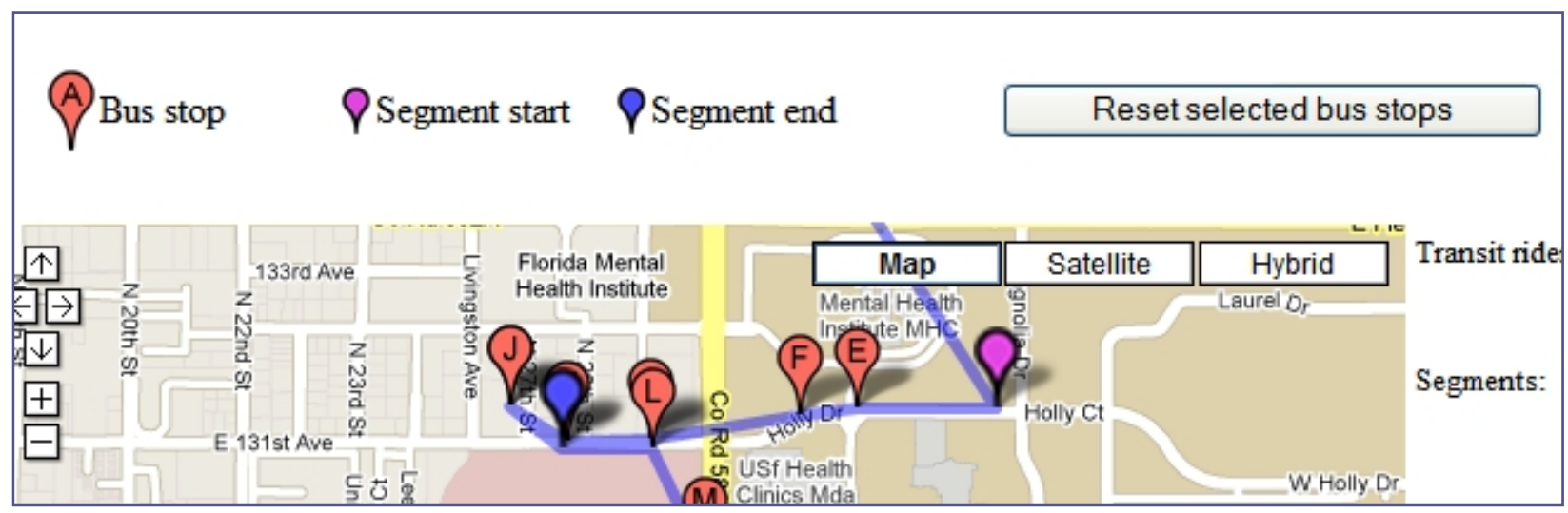

Figure 35 - New Button to Reset Selected Bus Stops While Creating New Trips

\section{Maintaining System Availability}

As with any preliminary system that is opened up to a larger audience for testing, the research team discovered some issues with TAD that were encountered as new testers were added to the system. The research team worked hard to quickly resolve them as they arose. On a few occasions, the TAD Web server froze up and became unresponsive. After this was brought to the research team's attention, the server was restarted and testing was resumed. The cause of the freezing is still unknown, as no error logs produced information which could lead to a diagnosis of the problem. As a result of these problems, the server instances supporting the TAD Web application and the TAD server software that communicates with the TAD cell phone application were split into two different instances. By isolating each of the software components, the 
research team will be able to better troubleshoot possible causes of the issue if it occurs again in the future.

Shortly after testing began with the first transit agency (MDT), the server was rebooted late at night in order to implements changes to the system. After the reboot, the MS SQL Server license was checked for validity and was found to be expired. This meant that TAD use and testing had to be put on hold while a new SQL server license was obtained.

Due to a higher volume of usage, warning messages regarding potential issues with frequent database connections began to appear in the Web server logs. When users access TAD through the Web application or the mobile application, connections to the database are acquired through a connection pool that has a limited number of connections available. If many users acquire those connections at the same time and the code is not properly configured to release those connections immediately after use, the number of available connections could become low enough to prevent TAD from working properly. To resolve this, code changes were made to ensure that all database connections were closed following use, and changes to the Web server were made to allow for more connections.

The informational TAD Web site also had small availability outages due to issues with the third party hosting service provider. The third party hosting provider was contacted and the issue was resolved.

The TAD development server experienced some problems, including freezes and automatic reboots, which were discovered to have been caused by a bad sector on the hard drive. This issue was resolved by replacing the hard drive. These problems did not impact the transit agencies testing TAD, since they were interacting with the production servers. 


\section{Chapter 5: Evaluating the Effectiveness of TAD on the Bus Riding Behavior of Individuals with Disabilities}

This chapter was authored by Arica J. Bolechala and is a summary of the work performed as part of her master's thesis in the USF Applied Behavior Analysis Master's Program [15]. The purpose of the research study discussed in this chapter was to determine whether the prompts given by the TAD would exhibit stimulus control over the participant's behavior of pulling the cord to stop the bus at the appropriate time and exiting the bus at the appropriate stop.

\section{Method}

\section{Participants and Setting}

Participants were three individuals with moderate mental retardation. The participants were ambulatory and had previous training on how to use public transportation but were unable to travel a new route on the bus independently. The first participant, Mark, is a 22-year-old male diagnosed with moderate mental retardation and Benin Congenital Hypotonia. He was traveltrained in 2008 and used the bus approximately five times per week, using the same bus and route each time. The second participant, Paige, is a 20-year-old female diagnosed with Down syndrome and moderate mental retardation. She was travel-trained in 2004 and used the bus approximately three times per week, using the same bus and route each time. The third participant, Clark, is a 25-year-old male diagnosed with Down syndrome and moderate mental retardation. He was travel-trained in 2004 and did not use the bus to access the community at the time of this study. The observations for baseline and intervention phases were conducted on the city transportations system in a metropolitan area. Training for using TAD took place by providing participants with behavioral skills training (BST) in a classroom setting.

\section{Target Behaviors and Data Collection}

The target behaviors marked for increase when using the TAD were (1) the participant will pull the bus cord within five seconds of passing the bus stop before his/her appropriate stop, signaling his/her bus stop, and (2) the participant will exit the bus at the appropriate stop. Data also were collected on if the participant looked at TAD when it vibrated, if TAD provided the prompts at the appropriate locations, if the participant asked people for directions to his/her location, and any observational data on what the participant was doing while on the bus.

\section{Procedure}

The results of the study were evaluated and presented using an A-B-A-B research design. The AB-A-B design represents an attempt to measure a baseline (the first A), a treatment measurement (the first $\mathrm{B}$ ), the withdrawal of treatment (the second $\mathrm{A}$ ), and the re-introduction of treatment (the second B). In other words, the A-B-A-B design involves two parts: (1) gathering of baseline information, the application of a treatment and measurement of the effects of this treatment, and (2) measurement of a return to baseline, what would happen when the 
treatment is removed and then again applying the treatment and measuring the change. If the TAD was not effective, in-situ training was provided and evaluated in a reversal design. As previously mentioned each participant had received travel training focusing on using a specific transit route.

\section{TAD Training}

Training for the TAD consisted of behavioral skills training (BST), which consisted of instructions, modeling, rehearsal, and feedback [16]. The verbal instructions described how the TAD operates, what prompts would be given by the TAD, and how to respond to the prompts. The modeling consisted of allowing the participant to hear the prompts and showing the participant how to respond to them. Rehearsal consisted of the participant hearing the prompts given by the TAD and following the prompts in a simulated setting. Verbal praise was provided to the participant, as was corrective feedback as needed. The training for the TAD was carried out in a classroom setting using a simple simulated bus setup. This setup consisted of chairs lined up to simulate bus seats and a string attached to the wall that simulated the cord that needed to be pulled on the bus. Following training, individuals participated in at least three assessments designed to evaluate the TAD.

\section{Results}

Figure 36 shows the results for each participant. Trials are presented on the $\mathrm{x}$-axis and steps completed ([1] participant pulling the cord within five seconds of passing the second-to-last stop, and [2] participant exiting bus at the correct stop) are presented on the y-axis. The data for all participants shows a low and stable baseline with a flat trend at zero when the participant was not carrying the TAD. After intervention (i.e., the participant carrying the TAD), the first post- training shows a level change to two, which remains high and stable throughout the phase. In other words, these participants correctly performed steps one and two while they were carrying the TAD. During the reversal to the second baseline (i.e., no TAD use), the graphs again show low and stable data with no trend at zero. Data from the second post-training (i.e., carrying the TAD again) show a level change to two, which again remains high and stable throughout the phase. 

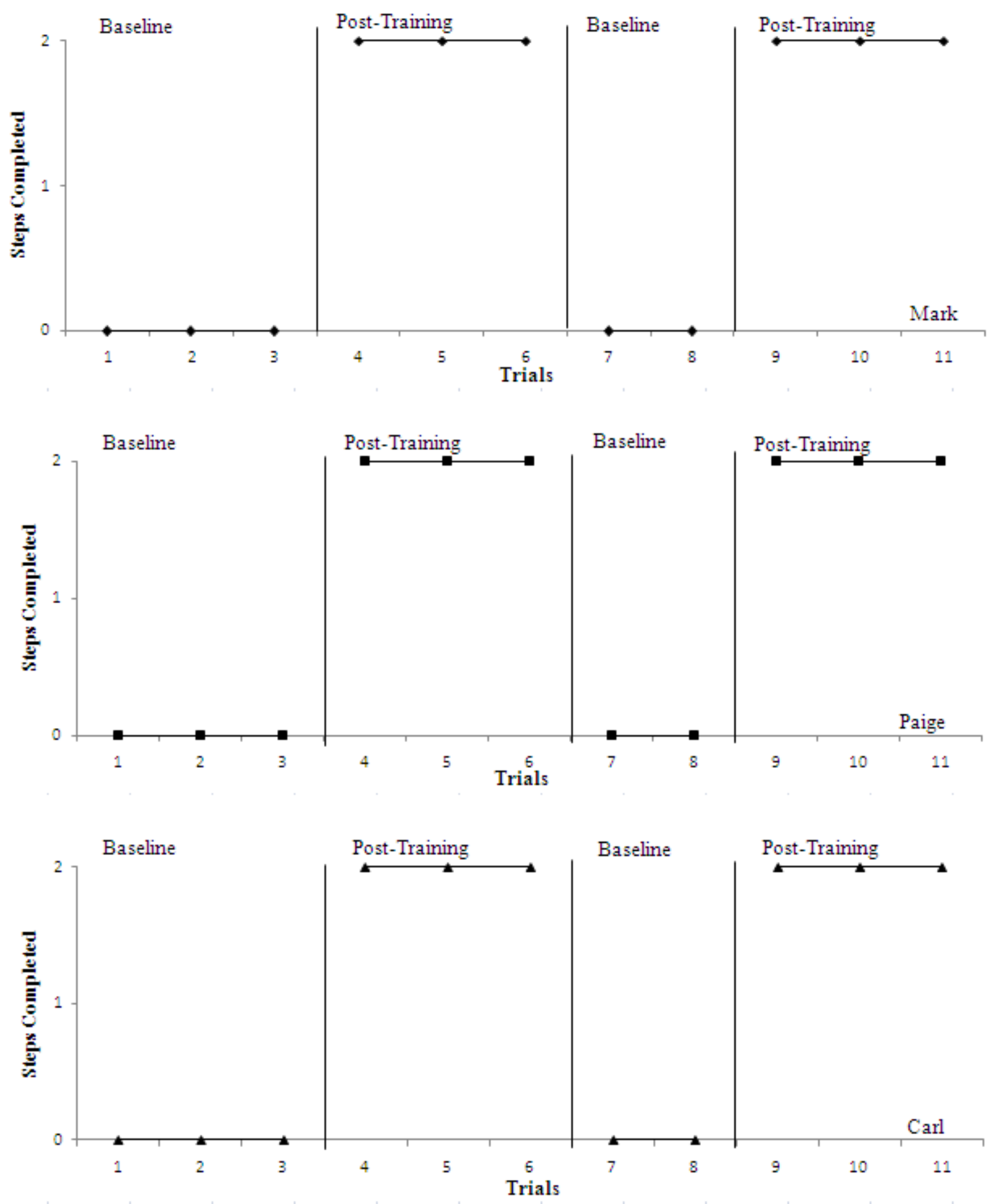

Figure 36 - Combined Baseline and Post-Training Results of TAD Trials 


\section{Discussion}

The current literature shows that with the advancement of technology, devices can be created to assist individuals with disabilities to increase their independence. The least researched and developed area is independent travel in the community. This study provides supporting evidence that the TAD is an effective device for prompting individuals to pull the cord to indicate their stop and to exit the bus at the appropriate location. During both baseline phases, without the TAD participants did not pull the cord to indicate their stop at the appropriate times. In this study, research assistants unknown to the transit rider rode the bus to ensure the participants' safety if they did not exit the vehicle at the correct stop. If the transit rider did not pull the cord for the correct stop within the designated timeframe, the research assistant intervened and pulled the cord to make the bus stop at the correct stop. A second research assistant, known to the transit rider, was waiting at the bus stop so they could step into the bus and tell the participant to exit the bus and come with them. Without these safety measures put into place for this study, the bus would not have stopped at the destination stop and the participant would not have exited the vehicle. Therefore, without TAD, the probability that an individual will miss his/her stop and ultimately get lost is increased. With the prompts given by the TAD, the participants were able to pull the cord and exit the bus at the appropriate locations. This was illustrated by one participant in particular, Mark. On the very first trip during baseline, Mark asked the bus driver for assistance, which was permitted because in the natural environment it is highly likely that people ask for assistance while on the bus. The bus driver told Mark the incorrect bus stop. Due to this information, Mark got off the bus at the wrong stop and had to be picked up early.

The TAD has the potential to afford mobility for individuals with disabilities who desire to travel independently by giving them a tool that will assist them in finding the places to which they want to travel. It also provided the individuals in the study with more confidence and assurance during their trips. During baseline assessments, all of the participants expressed concerns about traveling without the TAD to unknown locations. One participant, Paige, stated during every baseline trip, "I don't know where that is." However, once she was given the TAD, she did not express any concern about the location to which she was asked to ride the bus. Social validity surveys collected from the parents of the participants before and after the study show that before using the TAD, parents were not confident to somewhat confident in their child's ability to get off the bus at familiar and new bus stops. Surveys given to the parents after the study showed that when the participants used the TAD, parents were confident to very confident in their child's ability to get off the bus at both familiar and new bus stops. Parents also stated that they would like their child to use the TAD again when using public transportation.

Although this study shows good evidence in support of the use of an assistive device to gain independence in the community, there are some limitations to this study related to generalization. First, all participants in this study were diagnosed with the same level of mental retardation. The results from the study show favorable outcomes for these three participants with moderate mental retardation; however, more research needs to be conducted with a more diverse population to determine if TAD is a device that can be used by a more diverse 
population. Second, all participants previously had been travel-trained to use the local bus system. One of the parameters for selecting participants for this study was that all participants had to have been trained to use the city bus. While the years between the time when each of the participants was trained to the beginning of the thesis study varied among participants and the frequency of using the bus regularly varied, they all previously were taught a specific set of skills to be able to use the city bus to access frequently taken trips. This study did not consider whether individuals who were not travel trained would be able to use TAD as successfully as those who had been travel trained.

Additional studies are needed to be able to assess what level of independence that individuals using this device will experience. The current study assessed only how the participants responded to TAD when it was already activated and the bus trip had been selected and planned via the TAD Web site. In order for the TAD to provide prompts, the phone must be programmed with the personalized itinerary of bus stop locations via the TAD Web site, the application on the phone must be turned on when using the TAD, and each trip segment must be selected on the phone once the TAD application on the phone has been turned on. Future research should focus on if individuals with disabilities can use the Internet to access the TAD Web site to input bus trips into the system, access the TAD application on a cell phone, and select the desired bus trip. These studies would help clarify who will be able to use the TAD independently and how much assistance individuals might need to be able to use it. 


\section{Chapter 6 - Conclusions and Recommendations}

\section{Discussion of the Results}

This research project successfully deployed and tested TAD with BCT, MDT, PSTA, and SCAT. The report documents the level of logistical and technical effort required to deploy TAD and reports on the results of testing TAD at these agencies. The project demonstrated that TAD can function in cities beyond the initial testing area of Tampa by using commercially available GPSenabled cell phones and existing datasets formatted in the GTFS format from transit agencies.

\section{Implementation of TAD to Serve Transit Riders}

Implementation of this research will be the operation of the TAD system to serve transit users, particularly novice riders, seniors, tourists, and individuals with disabilities. To achieve that objective, USF's Division of Patents and Licensing has licensed TAD to Dajuta, a Florida company that will handle the daily operation and support of the TAD system and train travel instructors to use TAD with their trainees. This company will resolve various technical support issues as they arise, including distributing application updates, and will maintain a direct relationship with travel trainers using TAD with their clients. It is anticipated that USF will need to facilitate the initial transition of TAD to the third party for its launch as a service and will also continue to be involved in the development of TAD in order to address research questions as they arise.

TAD was cited in the 2009 Florida Governor's Commission on Disabilities Report as a Proposed Implementation Strategy to eliminate barriers to pedestrians with disabilities and make bus travel accessible for people with disabilities to facilitate access to mass transportation services (http://dms.myflorida.com/other programs/governor s commission on disabilities).

Dajuta will work with transit agencies in Florida to implement TAD for their customers to address this need.

The flowchart in Figure 37 can be used by transit agencies in order to determine the steps they would need to take to deploy TAD to their customers. 


\section{Acronyms:}

TAD = Travel Assistance Device

GTFS = General Transit Feed Specification

GPS = Global Positioning System

ESPA = Easter Seals Project ACTION

$\mathrm{API}=$ Application Programming Interface

$\mathrm{ETA}=$ Estimated Time of Arrival

Possible Funding Sources:

Federal Transit Administration's New Freedom Program (5317) (http://www.fta.dot.gov/funding/ grants/grants_financing_3549.html)

Commercial Partner:

Dajuta

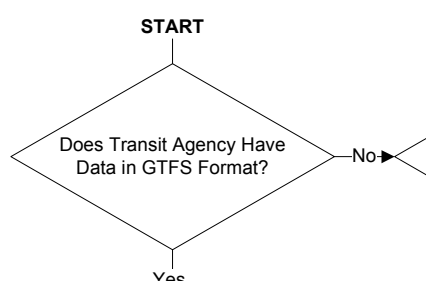

Yes

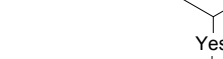

Yes

Aust Put Its Dat

GTFS Format to Use TAD.

More info here:

http://maps.google.com/help/maps/transit partners/participate.html

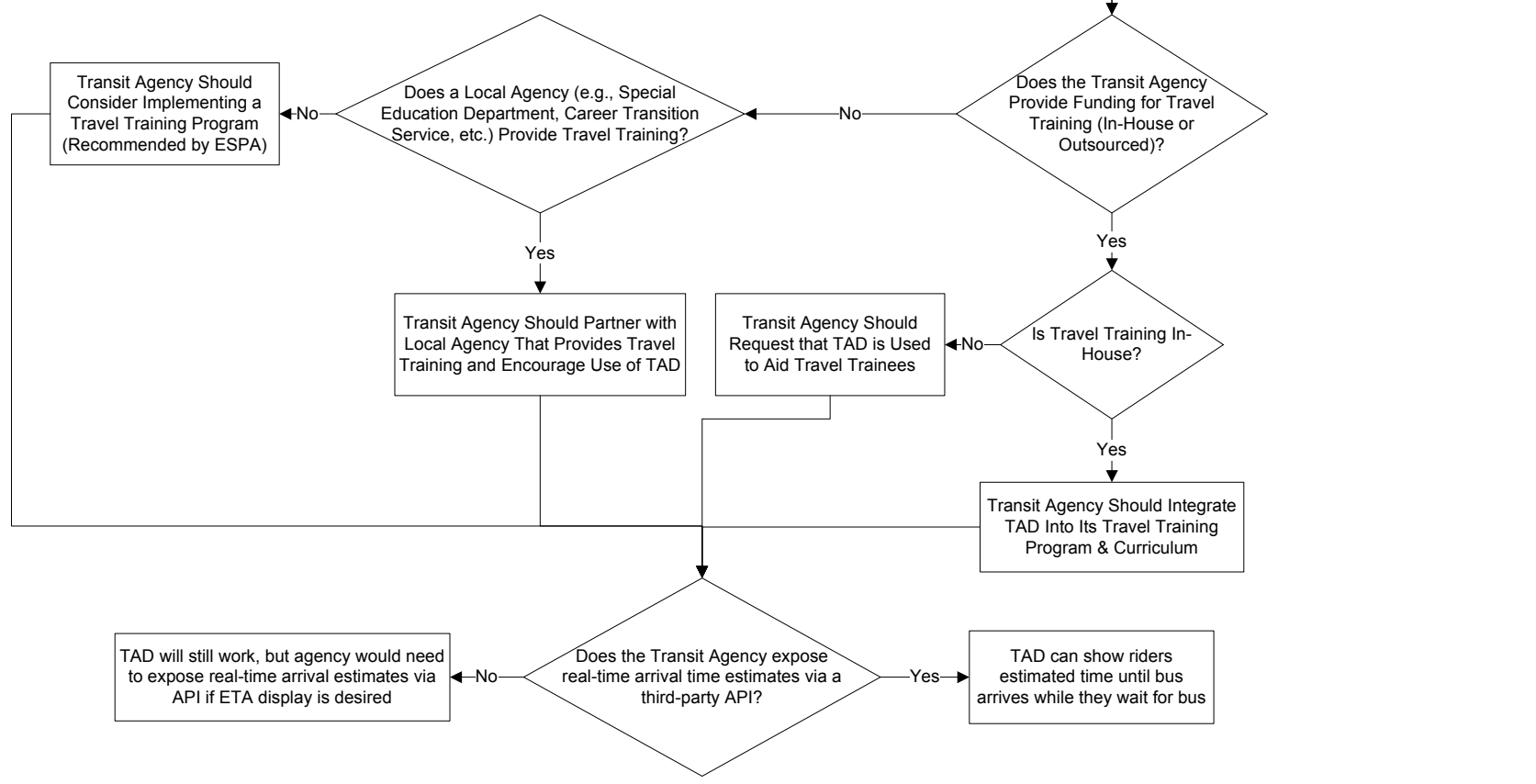

Figure 37 - Suggested Steps to Make TAD Available to Transit Customers

\section{Possible Funding Sources for TAD Implementation}

A sustainable funding source will need to be identified to support the long-term operation and success of TAD at transit agencies. The New Freedom Program (5317) from the Federal Transit Administration aims to provide additional tools to overcome existing barriers facing Americans with disabilities seeking integration into the workforce and full participation in society (http://www.fta.dot.gov/funding/grants/grants financing 3549.html). Since the lack of transportation is a key barrier that prevents many individuals with disabilities from being employed, the New Freedom grant program seeks to reduce barriers to transportation services and expand the transportation mobility options available to people with disabilities beyond the requirements of the Americans with Disabilities Act (ADA) of 1990. State and public bodies are 
eligible designated recipients of New Freedom funding. Capital and operating expenses for new public transportation services and new public transportation alternatives beyond those required by the ADA act that assist individuals with disabilities are eligible funding activities under the New Freedom program.

As part of the Global Travel Training Community Web site, Easter Seals Project ACTION has created an online database of travel training funding sources that are currently being used by transit agencies to provide travel training services to their customers (http://www.communityzero.com/gttc?go=165.5440). It is suggested that transit agencies review the funding sources listed here, since some of the funding sources for travel training may also be able to support TAD.

\section{Recommendations for Future TAD Deployments - Partnering with Travel Training Programs}

The research team invested a significant amount of time working with multiple transit agency staff members who do not conduct actual trips as part of their daily activities, unlike travel trainers who spend the majority of their workdays in the field providing one-on-one instruction to transit trainees. Although staff at all transit agencies contacted were very enthusiastic about the TAD concept and agreed to its potential in moving riders from paratransit to fixed-route, establishing a partnership between CUTR and these transit agencies was a very lengthy process. In absence of a travel trainer, the research team was directed to the agencies' paratransit coordinators, marketing specialists, customer service providers, and technology programmers/analysts.

The research team has seen firsthand the value of travel training while communicating with parents and trainees over the past few years during several projects including field tests with TAD. Studies cited in this report also state that travel training has proven to be a valuable tool to achieve mobility independence for transit riders while reducing agency operating costs. That independence encourages involvement in the community and that defines the quality of life and the wellbeing of the individual. While preparing for this study and searching for partners from different Florida transit agencies, the research team noted that the term, travel trainer, is unfamiliar to some staff and that at some agencies little or no emphasis is placed on the promotion of the travel training program on the transit agency Web site or via paper handouts. However, both HART and BCT do include information about their travel training programs, which appear to be in high demand, on their respective Web sites.

Successful use of TAD at a transit agency will likely require a local champion, at least in the preliminary stage, to coordinate with early users of TAD at the agency. HART's travel trainer functioned as this champion in the early TAD tests at HART. Additionally, having the buy-in of top level management seems to be a necessary component to testing $\mathrm{TAD}$, especially at agencies that do not have dedicated travel trainers. If an agency does not have dedicated travel trainers, then an employee from another department, who does not travel on the transit system as part of 
his/her normal job duties, must take time away from their normal job duties to go out in the field and test TAD.

Based on these findings, and the results of the human behavior analysis study detailed in Chapter 5 , the next step in the evolution of TAD is the integration of TAD within the travel training curriculum. The travel trainers at HART and BCT, as well as other travel trainers from agencies around the country, have expressed interest in using TAD with their clients. HART's travel trainer has said that he believes TAD will help expedite the training process, which would allow him to train more individuals. BCT's travel trainer, as well as many other people, has indicated that TAD would be useful to many individuals beyond those with cognitive disabilities, including those with visual impairments. A transit rider with physical disabilities has told the research team that she would benefit from TAD when her wheelchair is secured on the bus, since she often cannot see out the window to identify when she should request a stop due to the positioning of her wheelchair. Future work should examine current travel training curriculums and education methods and provide guidance to travel trainers on the use of TAD within their training sessions.

Since TAD is useful to many different individuals, both with and without different types of disabilities, the specific integration of TAD into the curriculum may differ depending on the trainee's capabilities. Easter Seals Project ACTION and the Association of Travel Instruction should be involved in this effort due to their expertise in the travel training field. Given the success of the study detailed in Chapter 5, additional behavior analysis research could provide valuable information to inform these efforts. Additional specific areas of recommended future behavior analysis research are discussed in a later section in this chapter.

The availability of adequate training and tutorial materials, as well as a user interface design for Web sites and mobile applications that anticipate various types of use by different users, also has proven important to aid new TAD users. The research team created a demo version of the TAD mobile application which was used to show participants how TAD functioned in the study described in Chapter 5. A trainer is able to trigger the Get Ready and Pull the Cord Now alerts in a classroom setting by pressing a button on the phone to show trainees what to expect from the mobile phone application. This demo application allows TAD education to take place before actually boarding a bus. However, teaching remote transit agency staff how to use the TAD proved more complicated. Based on the research team's experience, remote users tended to skip written instructions and jump straight into using the Web and mobile applications. However, the online video that the research team created to help users update the mobile phone application seemed to work well as a fast educational tool. Future work could include providing additional short instructional videos on the TAD Web site that quickly orient a new user on various TAD functions. These videos could be integrated into the actual trip planning Web page itself when possible to quickly address the major uses of the Web site and allow the users to explore frequently asked questions. 


\section{Suggested Future Improvements to the TAD System}

A variety of individuals used TAD during field tests, and a number of observations were made by both the field testers and the research team. These observations led to the development of a list of suggested improvements for TAD that should be examined in future research projects to help make TAD easier to use for both travel trainees and transit agencies and also easier to maintain by system administrators. Some suggested improvements recommend that transit agencies improve the quality of data in their GTFS feed. A short description of each of these potential improvements follows, prioritized by the research team to achieve the long-term success of TAD.

\section{TAD Mobile Application}

High-priority - Contributes to the long-term successful operation of TAD

1) Examine different application settings, including larger radii for the bus stop detection algorithm, on Pro 200 and Pro 700.

TAD must eventually be transitioned to run successfully on new cell phone models, since the Sanyo 7050s devices used for TAD field tests have been discontinued. While the Sanyo 7050s supported GPS refresh rates of once per second, it appears that the Sanyo Pro 200 and 700 phones support only GPS refresh rates of once every four seconds. Future work should investigate whether the distance thresholds used to trigger the Get Ready and Pull the Cord Now! notifications need to be adjusted to account for this reduced refresh rate, or adjusting the application settings could possibly increase the GPS refresh rate.

2) Create an Android version of TAD to reach transit riders on Verizon and TMobile and additional users on Sprint-Nextel and AT\&T networks.

As discussed in Chapter 2, creating an Android version of the TAD mobile application may be the best way to reach transit riders on Verizon and T-Mobile networks. An Android version of TAD also would reach smart phone users on the Sprint network and Nextel and AT\&T networks. Android has recently surpassed Apple's iPhone and RIM's Blackberry platforms in U.S. market sales to become the most popular smart phone platform in the U.S. (http://www.npd.com/press/releases/press 100804.html). From the research team's experience, there seems to be less variation among cell phone platform implementations on different manufacturers' devices on the Android platform than on the Java Micro Edition (Java ME) platform. Therefore, implementing an Android version of TAD, instead of porting the Java ME version of TAD to multiple different Java ME phones, may be a more cost-effective way to reach a significantly larger number of potential TAD users. Additionally, smart phones are significantly dropping in price and becoming more popular across many socio-economic demographics. Android-based smart phones also offer useful features such as a TextTo-Speech application programming interface (API) which would allow a mobile application to read text to a user. However, given the rapid changes in the telecommunication industry, these options must be re-evaluated at the time of implementation so that the most recent state-of-the-art technology in mobile software 
application development is considered. Additionally, it may be difficult for individuals with certain disabilities, such as visual impairments, to use touch-screen devices. Since most smart phone platforms, including Android, typically feature touch screens, potential TAD users should be consulted to ensure that a smart phone version of TAD remains usable and practical for all users.

\section{Low-priority - Feature Enhancements for TAD Mobile Phone Users}

1) Display distance in non-metric units (e.g., miles) in the mobile application. In the current TAD mobile application, the remaining distance to the destination stop is shown in meters, since this is the default unit for the GPS system. However, non-metric units likely are easier to relate to for the majority of the U.S. population. Therefore, future versions of TAD may include the ability to display distance in non-metric units, as well as a preference that would allow the user to select either metric or non-metric units. The distance display could be dynamically varied so that miles are displayed when the user is a large distance from his/her destination, and this display would change to feet when the user is much closer to the destination. By changing the distance display units dynamically, TAD would make it easier for users to understand their distance from the destination stop.

2) Add menu item and screen for updating the TAD mobile application. Currently, the TAD mobile application checks to see if an updated version of the TAD mobile application is available for download each time it is started. If an update is available, it will automatically open the browser and begin to download the update. This occasional change in the mobile application flow may confuse some transit riders who are accustomed to TAD functioning the same way on startup when updates are not available. Future TAD improvements could include a menu item that will check for the update and, if an update is available, ask the user if they would like to update TAD. This method would be used instead of the automated launch into the update when the TAD mobile application starts, so that the update is applied only if specifically directed by the user. For transit users with disabilities, the update process could then be supervised by a travel trainer or parent/guardian to ensure that TAD is working normally after the update.

\section{3) Add the route number to the mobile application display for agencies that do not include route numbers in their GTFS headsign data.}

Currently, if the transit agency populates the headsign field in its GTFS data, this field is what is shown to the user on the cell phone to help him/her board the correct bus for the trip while waiting at the bus stop. However, certain agencies, such as HART, do not include the route number in their GTFS headsign information. Therefore, even though the route number is displayed on the headsign of the bus, the route number does not currently show up in the TAD mobile application. By adding the route number to the mobile application display, the transit rider can match the cell phone display to the 
approaching bus headsign to make sure they are boarding the correct bus. Alternately, agencies like HART could modify their headsign field data to include the route number.

\section{TAD Web site}

\section{Low-priority - Feature Enhancements for TAD Web site Users}

1) Add the ability to assign a travel trainer to a specific agency through the TAD Web site for bus stop modification.

The permission to modify bus stop locations using the TAD Web site bus stop editing tool is currently limited to system administrators. Therefore, transit agencies interested in modifying bus stop locations through the TAD Web site need to be assigned the administrator role in TAD. Since system administrators can view the entire TAD system of users (i.e., users for all transit agencies), transit agencies are then provided access to other administrator-only features such as editing bus stop data for all agencies, creating new users, removing user roles, viewing all user information, etc. To limit the scope of access to the system for transit agencies and travel trainers, users having the travel trainer role could be allowed to modify bus stop locations, but only for the transit agencies whose data they have permission to modify. This feature requires some modifications to the system, including the ability for TAD system administrators to assign a travel trainer to a transit agency. However, this feature should not limit travel trainers to planning trips on a single transit system, since often travel trainers must make connections for trainees with multiple transit agencies, especially in metropolitan areas with several transit service providers.

2) Add the ability to audit bus stop editing by Web site users, with ability to roll back changes.

Once more users with the travel trainer role are given permission to edit the bus stop locations for an agency, it is desirable to have an auditing system that records which user made an edit and the date and time the edit was made. This feature would provide full auditing capabilities, which would allow all users with the administrator or travel trainer roles to see who has made edits to bus stops, as well as allow the system to roll back changes to earlier versions of the bus stop location if a user makes a mistake and incorrectly modifies a bus stop.

\section{3) Add automated trip planning capabilities to the TAD Web site}

Currently, the TAD Web site allows the user to specify the boarding and exiting stop for each route where the transit rider should get on and off the bus. However, the Web site does not provide automated trip planning based on the user's starting location and destination location. In other words, to plan a trip for TAD the Web site user must know each boarding and exiting stop, the routes the rider must use, and the transfer locations from one route to another, since the Web site does not automatically determine this information. This design originated from requests by travel trainers for fine-grained control over the boarding and exiting bus stops. Fine-grained control allows travel trainers to plan personalized trips and take into account specific disabilities and safety 
considerations, such as planning a trip to avoid having the trainee cross a street. However, some transit riders, including those without disabilities, would benefit from an automated trip planning feature of TAD which would automatically determine bus stops to board and exit and routes to travel based on a starting and ending location. Currently, TAD users can use the Google Transit automated trip planner, the agency's proprietary automated trip planner, or internal tools within the transit agency's information system to automatically plan a trip, and then input this information into the TAD Web site. Future work should examine integrating automated trip planning directly into the TAD Web site to avoid these extras steps. The open-source project, OpenTripPlanner (http://opentripplanner.org/) provides automated transit trip planning functionality which could be integrated into the TAD Web site. However, the TAD Web site should retain the capability of editing stop boarding and existing locations, even after automatically planning a trip, to allow travel trainers to plan trips for TAD based on the specific needs of trainees.

\section{4) Display trip direction when searching for trips on the TAD Web site if direction is not included in agency's GTFS headsign field.}

On the Create a new trip screen on the TAD Web site, the Trips dropdown box may list multiple trips with the same headsign value if the agency has not included the directional information in its GTFS headsign data. For some trips, some transit agencies do not include the direction of the trip in the headsign field in their GTFS feed. For example, most of HART's GTFS trips show two trips in the opposite direction of service, such as South to Marion TC and North to University Area TC. MDT typically follows this convention, with most trips labeled such as 21 - Northside and 21 - Downtown Miami. However, for other trips, MDT provides the same headsign value for both directions of service, 243 - Seaport Connection. Since only one headsign value is given for a route, users planning trips for MDT on the TAD Web site would see two trips to choose from with the same name. Further discussion with transit agencies is required to determine if this labeling is a result of different route configurations, such as loops, which have a single direction (e.g., clockwise), or other route traits. Potential modifications to the TAD system to prevent showing duplicate trip names for different trips include requesting that the transit agencies include the direction either in the headsign field or in the trips.txt GTFS file, which would distinguish direction difference as an o or 1 (i.e., outbound or inbound). Alternately, the agency could append data fields outside of the GTFS specification to the GTFS data, which could be consumed specifically by TAD for TAD features. However, if human-readable trip directions are a universal problem for all transit applications, potentially consuming the agency's GTFS data, it is recommended that the agency provide a solution within the GTFS specification so all applications can benefit from the improvements. 


\section{TAD Database Toolkit}

\section{Low-priority - Feature Enhancements}

\section{1) Create a validation tool that would be executed prior to importing new GTFS}

data feeds into TAD.

As discussed earlier in the report, while the GTFS defines a variety of field names for formatting transit agency data, it does not specify more exact data details that are required for a database that actually holds data from a transit agency GTFS feed. Therefore, a TAD Feed Validator tool that is executed on a new GTFS feed prior to import into the TAD database would be useful for TAD system administrators. This tool could provide a simple report based on the current data fields in the TAD database that would verify that data types, sizes, and formats in the transit agency's GTFS data are compatible with the current design of the TAD database. If there are any incompatibilities, the tool would create a short list of incompatibilities that could be used by $\mathrm{TAD}$ system administrators to adjust the database schema as necessary to accommodate the new transit data. Typically, it is not anticipated that a transit agency would need to make any modifications to its GTFS data. Instead, the TAD database would be adjusted to make it compatible with the new GTFS dataset.

\section{Recommendations for Future Human Behavior Analysis Research using TAD}

The human behavior analysis study summarized in Chapter 5 provides supporting evidence that the TAD is an effective device for prompting individuals to pull the cord requesting their bus stop and to exit the bus at the appropriate location. Without the TAD, study participants did not pull the cord to exit the bus at the appropriate times, which increases the probability that an individual would miss his/her stop and ultimately get lost. When the participants did carry TAD, the prompts given by the TAD enabled all of the participants to pull the cord and exit the bus at the appropriate locations. The benefits of TAD were illustrated by the experience of one participant in particular. On the very first trip when he was not carrying the TAD, he asked for assistance from the bus driver, who told him the wrong bus stop, and he exited at this incorrect stop.

TAD has the potential to afford independence for individuals with disabilities who desire to travel independently by giving them a tool that will assist them in finding the places they want to travel. It also provided the individuals in the study with greater assurance and confidence during their trips. All of the participants expressed concern when traveling without the TAD to unknown locations. One individual who did express concern without TAD showed no indications of concern when traveling with TAD. Social validity surveys collected from the parents of the participants before and after the study show that before using TAD, parents were not confident to somewhat confident in their child's ability to get off the bus at familiar and new bus stops. Surveys given to the parents after the study showed that when the participants used TAD, parents were confident to very confident in their child's ability to get off the bus at familiar 
and new bus stops. Parents also stated that they would like their child to use TAD again when using public transportation.

There are many areas of potential future work for human behavior analysis related to TAD. For example, an inclusion and exclusion criteria should be developed to determine who is an appropriate user of TAD as a travel trainee. Once this criterion is developed, future research could also help develop assessment tools and guidance for travel trainers so they can successfully evaluate a trainee (i.e., how to train the trainer). Additional testing with a more diverse population to determine if TAD can be used successfully by these populations is also desired. Additionally, since all participants in this study were previously travel trained, additional testing should be performed with individuals who are not travel trained. Additional tests to determine the ability of individuals to use other components of the TAD system, including portions of the mobile application, TAD Web site, and general cell phone proficiency, could also be performed. These studies could help clarify who will be able to use TAD independently and how much assistance individuals might need in using it.

\section{Other Research Needs}

Since TAD is a new type of application for public transportation, it opens the doors to many new ideas and potential uses. It is expected that many additional research areas will arise as TAD is made commercially available at many different transit agencies. Some of these potential areas of future work are listed below.

口 Development of a cost-benefit models for TAD for use by transit agencies (based on whether training programs are in-house or outsourced)

$\square$ Facilitate the exact funding process for transit agencies to implement TAD.

$\square$ Study how TAD could help different populations (e.g., sight-impaired, seniors, individuals with epilepsy, physically disabled, etc.). For example, the James A. Haley Veterans Hospital in Tampa has expressed interest in to explore teaming opportunities on how TAD might be utilized by veterans returning with multiple disabilities. TAD may need to be modified to effectively prompt certain populations to exit the bus at the right place.

$\square$ TAD could also be expanded to tourists and include location-based advertising which could generate income to the transit agency.

$\square$ If TAD is expanded to smart phones such as those with the Google Android platform, future research would be needed to understand if smart phone GPS is as accurate as the GPS on low-cost flip-phones which have previously been tested with TAD. 


\section{References}

[1] Easter Seals Project ACTION: Accessible Community Transportation In Our Nation (ESPA). Accessed July 62010 at http://projectaction.easterseals.com/.

[2] LaHood, Ray. "Easter Seals Project ACTION seeks better youth mobility.” June 30, 2010. Secretary of U.S. Transportation. Accessed July 62010 at http://fastlane.dot.gov/2010/06/earlier-today-i-blogged-about-older-americansand-livability-now-as-we-approach-the-20th-anniversary-of-the-americanswith.html.

[3] National Council on Disability. 2005. "The Current State of Transportation for People with Disabilities in the United States National Council.” Accessed June 2010 at http://www.ncd.gov/newsroom/publications/2005/pdf/current state.pdf.

[4] Wolf-Branigin, Michael, and Karen Wolf-Branigin. 2008. "The Emerging Field of Travel Training Services: A Systems Perspective.” Journal of Public Transportation 11(3).

[5] Multisystems, Inc. 2002. "ADA Complementary Paratransit: A Decade of Innovation. Part 3 - Case Studies.” Easter Seals Project ACTION, Washington, D.C.

[6] Wagner, Dennis, and Jeff Delong. "Cities' paratransit services face cutbacks, fare increases." http://www.usatoday.com/. Ed. Brent Jones. USA Today, 1 Apr. 2009. Accessed July 62010 at http://www.usatoday.com/news/nation/2009-03-31dialaride N.htm.

[7] Bierman, Noah. "Cost of rides for disabled soars at T: Budget grows twice as fast as ridership.” Boston Globe, 28 Mar. 2010. Accessed July 62010 at http://www.boston.com/news/local/massachusetts/articles/2010/03/28/cost of ri des for disabled soars at $t /$ ?page $=4$.

[8] Wolf-Branigin, Karen, and Michael Wolf-Branigin. 2010. “A Travel Training Cost Benefit Model for People with Disabilities, Public Transportation Agencies and Communities." Paper presented at the Disability Forum: Accessibility for All, 12th Annual TRANSED International Conference in Hong Kong, sponsored by Easter Seals Project ACTION.

[9] Barbeau, Sean J., Philip Winters, and Nevine Georggi. 2008. "Travel Assistant Device (TAD) to Aid Transit Riders with Special Needs, Final Report." National Center for Transit Research, USF. Sponsored by the Florida Department of Transportation. Accessed May 2010 at http://www.nctr.usf.edu/pdf/77711.pdf.

[10] Barbeau, Sean J., Nevine L. Georggi, and Philip L. Winters. "Integration of GPSEnabled Mobile Phones and AVL: Personalized Real-Time Transit Navigation 
Information on Your Phone," Proceedings of the National Academy of Sciences' Transportation Research Board 89th Annual Meeting, Paper \# 10-2571. Washington, D.C., January 12th, 2010.

[11] Metro Magazine staff. February 2009. "Travel Software to Aid Disabled Riders." Metro Magazine. Accessed May 2010 at http://www.metromagazine.com/Article/Story/2009/02/Travel-Software-to-Aid-DisabledRiders.aspx.

[12] Google Maps - “Add your transit data to Google.” Accessed July 62010 at http://maps.google.com/help/maps/transit/partners/participate.html.

[13] Google Transit Data Feed project hosting. Accessed July 6, 2010 at http://code.google.com/p/googletransitdatafeed/wiki/FeedValidator.

[14] Goodwill, Jay and Holly Carapella. 2008. Creative Ways to Manage Paratransit Costs. University of South Florida: Center for Urban Transportation Research. Accessed July 8, 2010 http://www.nctr.usf.edu/pdf/77606.pdf.

[15] Bolechala, Arica J. July 2010. "Evaluating the Effectiveness of the Travel Assistance Device on the Bus Riding Behavior of Individuals with Disabilities.” Master's Thesis, Applied Behavior Analysis Program. Department of Child and Family Studies, College of Behavioral and Community Sciences, University of South Florida.

[16] Himle, M. B., R. G. Miltenberger, C. Flessner, and B. Gatheridge. 2004. "Teaching Safety Skills to Children to Prevent Gun Play." Journal of Applied Behavior Analysis 37, 1-9. 
July 2010

$\bar{\square}$

Appendix A

Solicitation letter to transit agencies and list of frequently asked questions

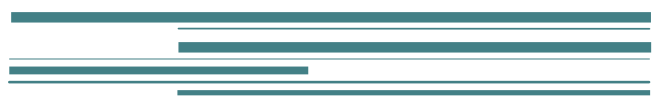




\section{Appendix A}
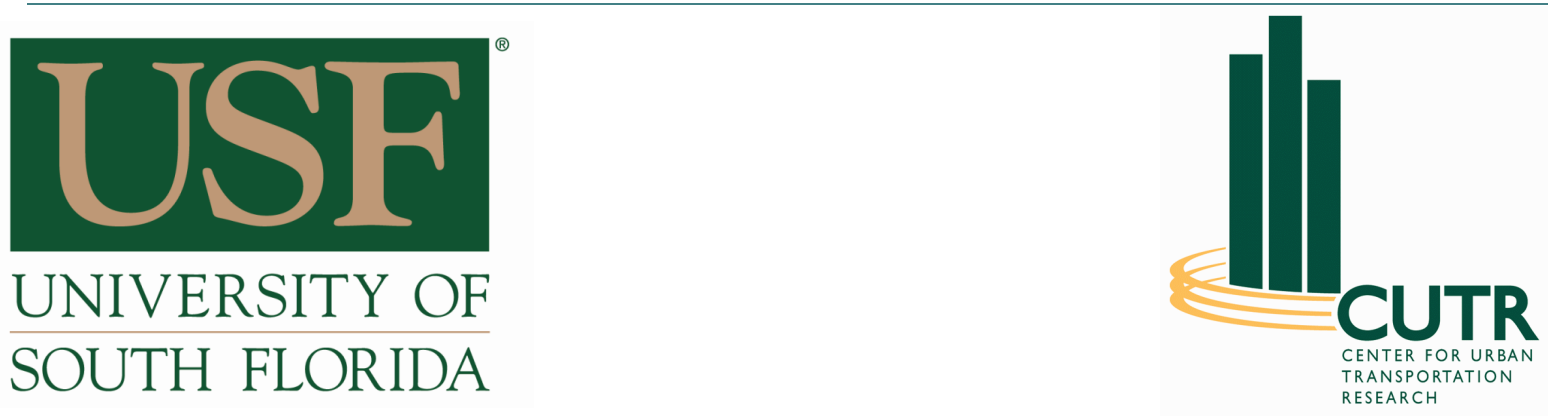

January 7, 2010

Dear Contact in Transit Agency ABC,

A Florida Department of Transportation (FDOT)-funded research project, the Travel Assistance Device (TAD), offers much promise for increasing accessibility, increasing transit ridership, and saving money for the transit agency. To help new and existing transit riders successfully navigate the transit system, transit agencies use a range of techniques including advertising trip routes, online trip planning tools, and travel trainers. TAD, a software navigation application for GPS-enabled cell phones, is a new tool that reduces the learning curve for transit riders by providing riders with personalized real-time audio, visual, and tactile prompts to aid them in their travels.

We are asking for your cooperation by allowing your agency's travel trainers to assist the Center for Urban Transportation Research (CUTR) in the deployment testing phase of this funded project.

We are not asking for funding.

Currently, CUTR, with sponsorship from FDOT (Research Project \# BDK85 TWO 977-12), is deploying TAD to transit agencies for field testing by travel trainers and transit riders. TAD was developed by the National Center for Transit Research at CUTR at the University of South Florida (USF) as part of a research project funded by FDOT and the U.S. Department of Transportation. While TAD was created to aid transit riders with special needs in order to increase their level of independence, any new transit rider can benefit from its service.

The current phase of the project seeks to evaluate (1) the level of effort required by a transit agency to make TAD available for their community, (2) changes in mobility and quality of life among TAD users, and (3) TAD performance and customer satisfaction with TAD from riders, parents/guardians, and transit agencies. In order to accomplish the set objectives of this research, transit agencies that have expressed interest in deploying TAD are asked to collaborate with CUTR to support pilot tests. 
We are asking you to commit your transit agency's travel trainers and information technology staff in providing data and travel trainers in field testing the TAD on your system to assist this FDOT-sponsored project. There are five areas where we need your help:

1) Have transit trainers use TAD as a regular part of daily trips to test quality of bus stop information, accuracy of TAD in that environment, etc. On average, we anticipate that testing TAD and reporting results back to the CUTR research team will take around 15-20 minutes per day, part of which will overlap with normal traveling duties of the travel trainer.

2) Provide bus stop inventory and schedule information in Google Transit Specification Format.

3) Have transit employees correct errors in any bus stop locations found through field testing through TAD's custom Web site application using Google maps.

4) Allow travel trainers to help coordinate recruitment and TAD testing with transit riders with special needs to provide feedback to the research team.

5) Provide feedback on system performance, user interactions, and system reliability to TAD research team.

As part of this effort, the CUTR research team will do the following:

1) Provide an online TAD demonstration for transit agency staff.

2) Monitor TAD and provide support during tests to resolve technical issues as they occur.

3) Provide transit agency travel trainers with access to Web page to easily plan their trips and modify bus stop locations.

We have attached a list of Frequently Asked Questions (FAQ) with answers to common questions regarding TAD testing. Hillsborough Area Regional Transit (HART) in Tampa has participated in TAD testing over the past two years as part of the initial TAD proof-of-concept project. A letter from Mark Sheppard, HART travel trainer, is attached that discusses his experience with TAD testing and his views regarding the time and effort required by the transit agency as well as the potential benefits to the agency for participating in a deployment of the TAD system.

If you have any other questions or concerns about participating in testing TAD, please feel free to contact me. I will follow up via a phone call in two weeks to discuss your agency's participation in the TAD field tests.

Sincerely,

Sean Barbeau

Research Associate, CUTR

4202 E. Fowler Avenue, CUT100

Tampa, FL 33620-5375

(813) 974-7208

barbeau@cutr.usf.edu
Nevine Labib Georggi

Research Associate, CUTR

4202 E. Fowler Avenue, CUT10o

Tampa, FL 33620-5375

(813) 974-9770

georggi@cutr.usf.edu 


\section{Frequently Asked Questions (FAQ) for TAD Testing}

\section{Does participation in TAD testing require any funding from my transit agency?}

No. The only commitment required from the transit agency is the time of staff members to help test TAD.

2. Since TAD is a software application for a cell phone, do we need to buy cell phones or cellular service to participate in TAD testing?

No. The CUTR research team will provide the phones and service for you to test TAD at your transit agency.

3. Why would my transit agency want to participate in TAD testing?

The research team expects the transit agency to benefit from the results of this project through testing a new technology that has the potential to decrease long-term paratransit costs by increasing the number of fixed-route transit riders, improving the state of their bus stop inventory through feedback from travel trainers and the TAD Web site Bus Stop Editor tool, and demonstrating a commitment to accessibility and support of transit riders with special needs to their patrons. Plus, TAD is useful for any new transit rider and can help lower the learning curve for public transportation, thereby increasing ridership.

\section{What data does our transit agency need to provide?}

Participation in TAD testing requires that your transit agency put their bus stop, route, and schedule data into Google Transit Feed Specification (GTFS) format and provide remote access data to the CUTR research team. Real-time access to automatic vehicle locator data is optional.

\section{What is Google Transit?}

Google Transit is a free online transit trip planner created and maintained by Google. To participate in Google Transit, your transit agency must put your schedule, bus stop, and route data into the Google Transit Feed Specification Format (GTFS).

6. What is the Google Transit Feed Specification (GTFS)?

The GTFS is a simple standard created by Google and the transit community which dictates how bus schedule, route, and stop data should be formatted. For an online system such as Google Transit or TAD to provide services to many different transit agencies, transit data for all participating systems must be maintained in a common format. TAD requires that the participating transit agencies provide their data in the GTFS format so that the data can be imported into the TAD system. More information on GTFS is available here at http://code.google.com/transit/spec/transit feed specification.html

7. Does my agency have to participate in Google Transit to be involved with TAD testing?

No. However, the CUTR team does recommend that you participate in Google Transit since Google Transit will provide additional vetting and quality control of your data in the GTFS format. Plus, you will get a free online trip planner for your customers, Google Transit.

8. How much time is required by the travel trainer or other personnel to participate in TAD testing? 
The travel trainer will be expected to use TAD as a regular part of daily trips to test quality of bus stop information, accuracy of TAD in that environment, etc. On average, we anticipate that testing TAD and reporting results back to the CUTR research team will take around 15 to 20 minutes per day, part of which will overlap with normal traveling duties of the travel trainer. If initial testing is successful, the travel trainer will be asked to help coordinate recruitment and TAD testing with transit riders with special needs to provide feedback to the research team. The travel trainer would then be required to successfully complete the USF Institutional Review Board free online training (approximately one to two hours) before any interaction between TAD and the general public. We expect TAD testing with transit riders to take an average of one to two hours per participant, and to test around 10 participants on each transit system.

\section{My agency doesn't have a travel trainer. What is travel training? Can we still participate?}

Travel training (also known as travel instruction) is short-term, one-to-one, intensive instruction designed to teach people with disabilities and seniors to travel safely and independently on fixed-route public transportation in their community. Individuals with disabilities and seniors learn travel skills while following a specific route, typically to a school or a job site. Travel instruction professionals must be able to determine how different disabilities affect a person's ability to travel, and they must develop appropriate methods to teach travel skills dependent upon individual needs, (from travelinstruction.org).

If your agency does not have a travel trainer, another person would be expected to complete the same tasks as required of the travel trainer to participate in TAD testing. More information on travel training from Easter Seals Project ACTION

http://projectaction.easterseals.com/site/PageServer?pagename=ESPA travel training

10. We have a bus stop inventory, but don't know how accurate it is. Can we still participate in TAD testing?

TAD uses a Global Positioning System (GPS)-chip embedded in the cell phone, along with the knowledge of the destination bus stop location, to provide the transit rider with real-time alerts. TAD is only as accurate as the bus stop inventory provided by the transit agency, since the proximity of the cell phone to the bus stop triggers the alerts to the rider. Generally, if the bus stops locations in the inventory are within 65 feet (i.e., 20 meters) of their true location, TAD should perform adequately. However, the better the accuracy of the bus stop inventory, the better TAD will perform. If you are unsure whether your bus stop inventory is accurate enough, please contact the CUTR team with your questions.

11. When will TAD be available to the general public as a service? The first step towards making TAD available to the general public in an area is to do some initial field testing to ensure the bus stop inventory is accurate and TAD works properly in the transit environment in that area.

\section{How much will TAD cost, and who will pay for the service?}


TAD is still emerging from research project status, so we do not have all the answers yet for how a commercial TAD system will operate. USF Division of Patents and Licensing is identifying potential commercial partners to ensure that TAD is a properly supported and successful service. The partner is likely to determine the appropriate business model - transit-agency sponsored (e.g., grants), user-fee supported, or combination.

\section{How can I help make TAD successful?}

The long-term success of TAD will likely be driven by three tasks:

a) Designing a user-friendly system that uses existing phone and communication networks technologies to deliver real value to customers;

b) Demonstrating to transit agencies that TAD is a useful and needed service that will help transit riders;

c) Identifying sources of funding for the transit agency to support the operational cost of TAD.

14. I'm interested in testing TAD in our city. What are the detailed requirements of my transit agency for participating in TAD testing?

a) Put your transit data into the GTFS format.

b) Assign/delegate a travel trainer or similar personnel within the transit agency to assist the CUTR research team in field testing TAD. If the agency does not employ transit trainers but outsources the service, a representative from the related service would be expected to complete the same tasks. This person would actively assist the project team with testing TAD as part of their daily trips to test bus stop accuracy, TAD performance in that environment, etc. and providing feedback to CUTR. If TAD is made available to the public on a limited basis, this person would assist in recruiting individual participants, securing parental consent if necessary, conducting participant orientation sessions, and helping to evaluate TAD performance on that transit agency and obtaining feedback from any participants. The travel trainer will also correct any observed errors for bus stop locations through the TAD Web page and provide feedback to CUTR research team.

c) As part of the research team, traveler trainers will participate and successfully complete the USF Institutional Review Board free, online training (approximately 1 to 2 hours). The USF Division of Research Integrity \& Compliance administers key research-related assurance and compliance programs required by federal and state agencies and programs for the conduct of research at USF.

d) Provide the following data (from the last year or two) on travel training to the CUTR research team: number of trainers, average number of trainees a month, average number of individuals on the waiting list to receive training, list of any partnerships with a transition program, new rider training, senior training, etc.

e) Provide free access to transit system for CUTR research team and field test participants/volunteers for the testing period. 
July 2010

$\bar{\square}$

Appendix B

Miami-Dade's GTFS Update Process

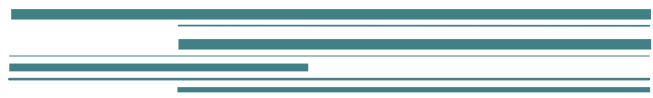




\section{Appendix B}

\section{Miami-Dade's GTFS Update Process}

Transit agencies often ask about the level of effort necessary to format their data in the GTFS format. Miami-Dade Transit provided a written description of its update process that must be completed each time it updated its transit data with a new markup.

The update process starts with an export of the agency's data from the Trapeze Export Tool for Google Transit Trip Planner, which is part of their scheduling software package provided by Trapeze. This tool requires Trapeze FX and one of Trapeze's Traveler Information Trip Planning Solutions

(http://www.trapezegroup.com/solutions/pt google.php). The following steps were documented by Miami-Dade Transit.

\section{Google Feed Validator via Python}

Place all Google Export files inside C: \GoogleFeed Launch cmd via Run.

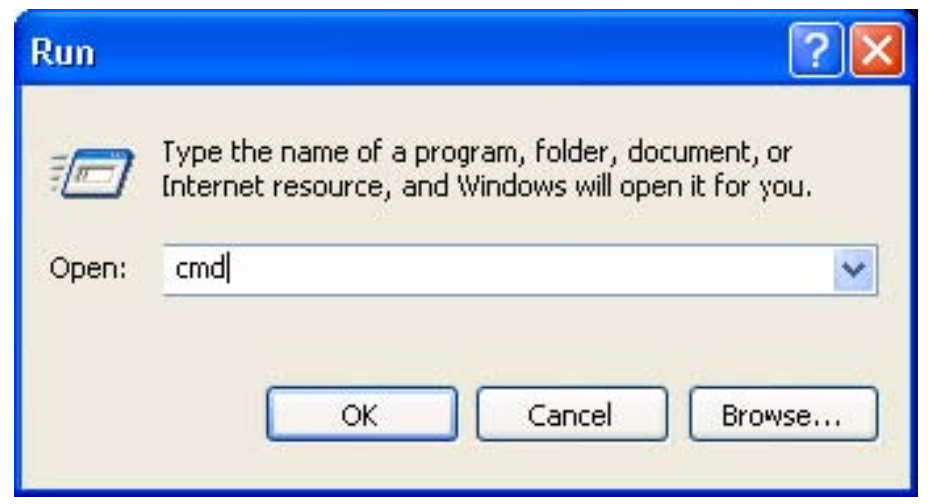

Type the information as screenshot below (it is -L [lower case L] not minus one). 


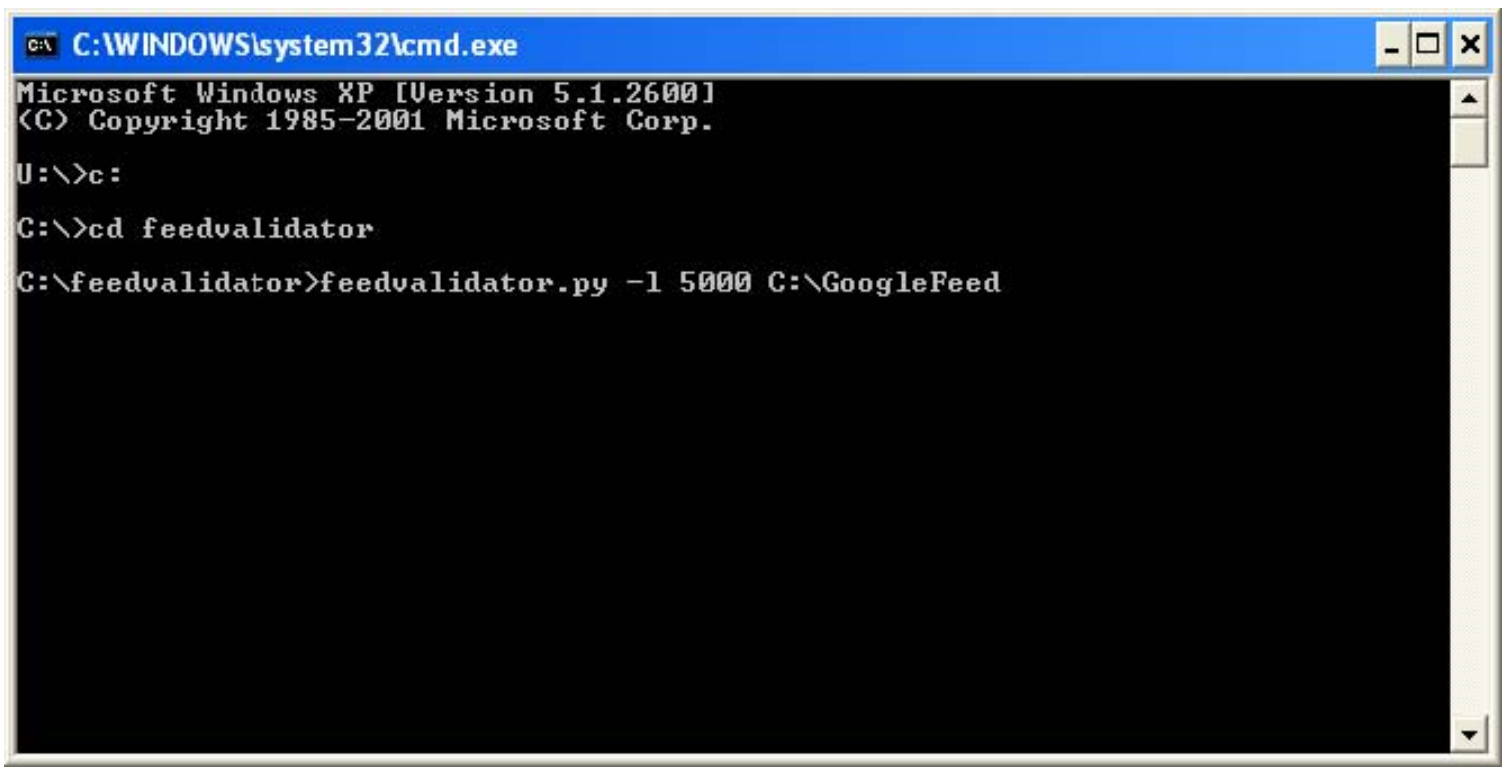

\section{Results: Validation Results to fix}

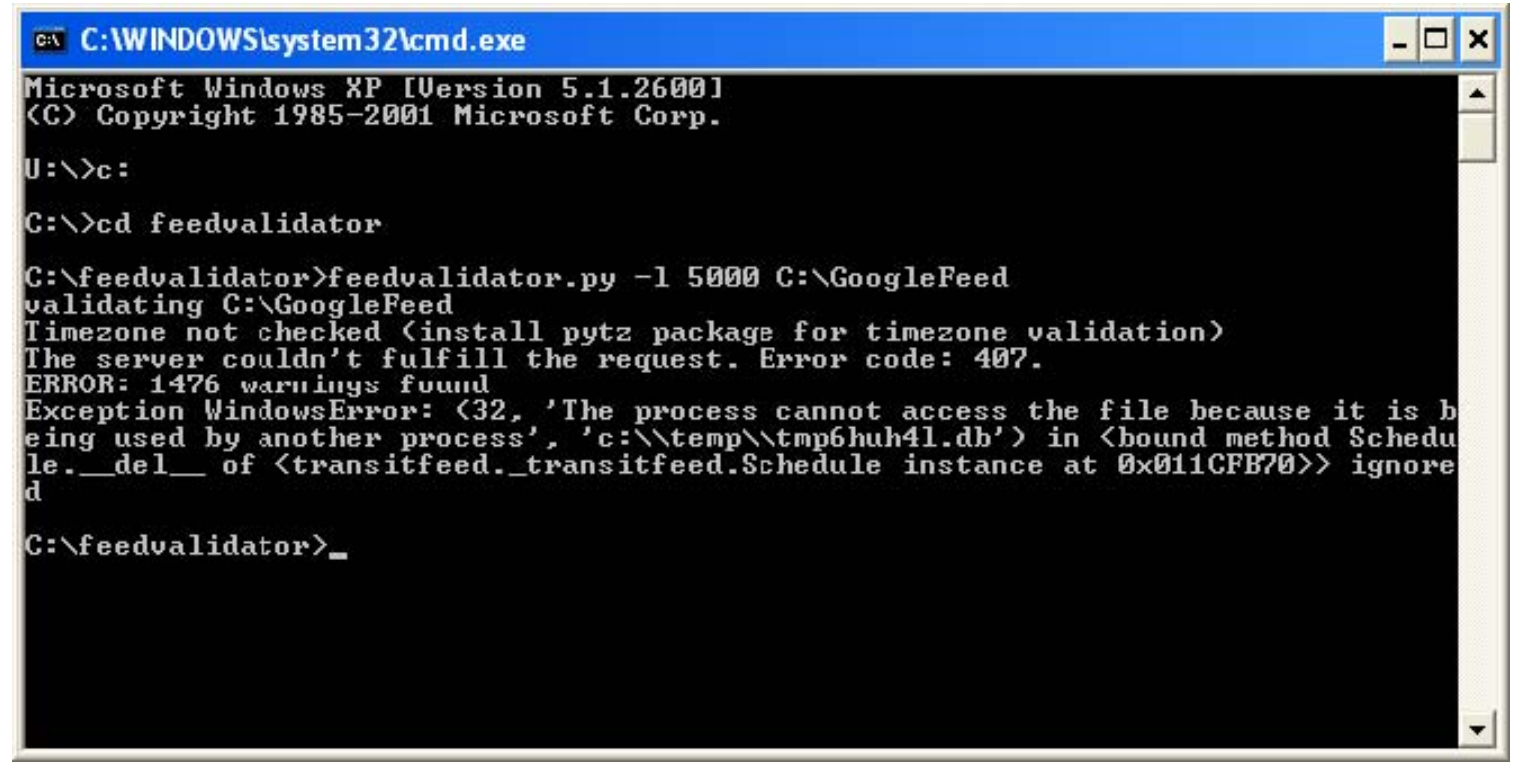

GTFS validation results for feed

$c: \backslash$ googlefeed

Agencies: Miami Dade

Transit Routes: 96 
Stops: 8365 Trips:

14248 Shapes: 420

Effective: June 27, 2010 to December 11, 2010

During the upcoming service dates Sun Jun 27 to Sat Jul 24:

Average trips per 5821

$$
\text { date: } 6626 \text {, on } 20
$$

service dates (Mon Jun 28, Tue Jun 29, Wed Jun

$30, \ldots)$ Least trips on a 3522 , on 4 service dates

(Sun Jun 27, Sun Jul 04, Sun Jul 11, date: ...)

The server couldn't fulfill the request. Error code: 407 .

Found these problems

\section{3 warnings}

1 Future Service

120 Invalid Values

447 Other Problems

199 Stop Too Far From Shape With Dist Traveleds

9 Stops Too Closes

1276 Too Fast Travels

1 Unknown File

\section{Warnings}

\section{Future Service}

The earliest service date in this feed is in the future, on June 27, 2010.

Published feeds must always include the current date.

Invalid Value (Change route color to a contrasting value; for these we change them to 'oooooo') 
Invalid value CoCoCo in field route_color The route_text_color and route_color should be set to contrasting colors, as they are used as the text and background color (respectively) for displaying route names. When left blank, route_text_color defaults to oooooo (black) and route_color defaults to FFFFFF (white). A common source of issues here is setting route_color to a dark color, while leaving route_text_color set to black. In this case, route_text_color should be set to a lighter color like FFFFFF to ensure a legible contrast between the two.

in line 14 of routes. txt

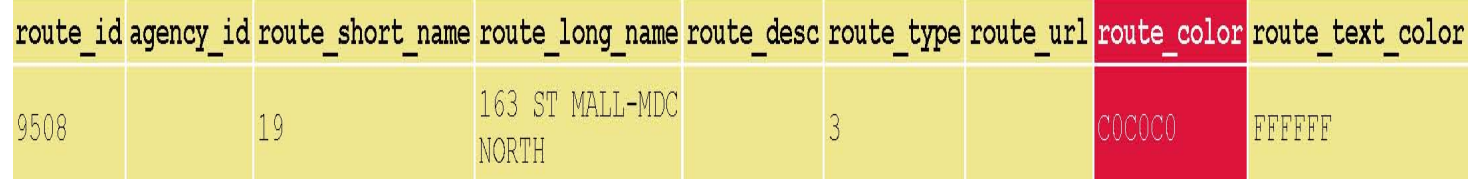

Invalid value A6CAFo in field route_color The route_text_color and route_color should be set to contrasting colors, as they are used as the text and background color (respectively) for displaying route names. When left blank, route_text_color defaults to 000000 (black) and route_color defaults to FFFFFF (white). A common source of issues here is setting route_color to a dark color, while leaving route_text_color set to black. In this case, route_text_color should be set to a lighter color like FFFFFF to ensure a legible contrast between the two.

in line 22 of routes.txt

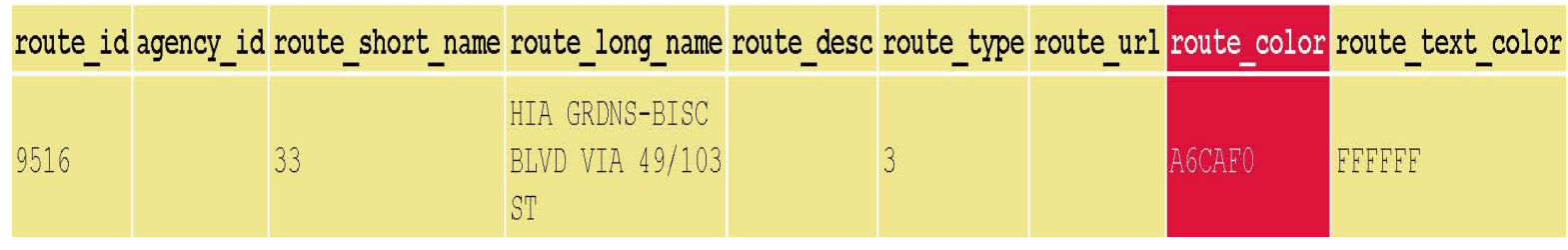

\section{Route short name and route long name cannot be the same for Google Transit (add an underscore to route long name to fix)}

Invalid value 79 ST MAX: MIA BCH-NORTHSIDE in field route_long_name route_long_name shouldn't contain the route_short_name value $\bar{e}$, as both fields are often displayed side-by-side.

in line 46 of routes.txt 
route id agency_id route short name route long name route desc route type route url route color route _text_color 954 79

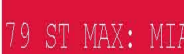

79 ST MAX: MAA
BCH-NORMHSTDE

008000

EFEEE

For the error below, you must look at the pattern in Trapeze for this route and find out what is wrong with it. Use the following query to translate tripid to route/pattern/direction:

SELECT ml.lineabbr, l.directionname, mp.pattern, t.fromtime, t.totime

FROM masterline ml, line l, masterpattern mp, trips $\mathrm{t}$

WHERE ml.lineid $=$ l.lineid

AND l.linedirid $=$ t.linedirid

AND t.patternid $=$ mp.patternid

AND t.tripid $=[$ TripId, Long $]$

Invalid value 15.1902 in field stoptimes.shape_dist_traveledFor the trip 2118959 the stop 363 has shape_dist_traveled=15.1902, which should be larger than the previous ones. In this case, the previous distance was 15.1902.

\section{Other Problem (change value of 34.536200 to something less than} 34.49500o directly in stop_times.txt)

In stop_times.txt, the stop with trip_id=2105254 and stop_sequence=112 has shape_dist_traveled $=34.536200$, which is larger than the max shape_dist_traveled $=34.495000$ of the corresponding shape (shape_id=90589)

In stop_times.txt, the stop with trip_id=2105255 and stop_sequence=112 has shape_dist_traveled $=34.536200$, which is larger than the max shape_dist_traveled $=34.495000$ of the corresponding shape (shape_id=90589)

\section{Warnings}




\section{Future Service (warning expected if you are validating upcoming instead of current line-up)}

The earliest service date in this feed is in the future, on June 27, 2010.

Published feeds must always include the current date.

\section{Stop Too Far From Shape with Dist Traveled (this warning is ignored)}

For trip 2108723 the stop "US 1@MM 98 SOUTH NEAR" (ID 9840) is 81730 meters away from the corresponding point (shape_dist_traveled: 47.580200) on shape 90709. It should be closer than 1000 meters. For trip 2108668 the stop "US $1 @$ MM 98 SOUTH NEAR" (ID 9840) is 81730 meters away from the corresponding point (shape_dist_traveled: 47.580200) on shape 90708. It should be closer than 1000 meters.

\section{Stops Too Close (this warning is ignored)}

The stops "SW 268 ST@SW 119 PL" (ID 443) and "SW 268 ST@SW 119 PL" (ID 448) are $0.00 m$ apart and probably represent the same location.

The stops "SW 136 St \& US \# 1" (ID 439) and "SW 136 St \& US \# 1" (ID 436) are $0.00 \mathrm{~m}$ apart and probably represent the same location.

The stops "Old Cutler Rd \& SW 57 Ave" (ID 438) and "Old Cutler Rd \& SW 57 Ave" (ID 437) are o.oom apart and probably represent the same location.

The stops "Vizcaya Station" (ID 182) and "Vizcaya Station" (ID 187) are o.oom apart and probably represent the same location. 


\section{Too Fast Travel (this warning is ignored)}

High speed travel detected in trip 2106282: NW 36 ST@NW 79 AV to NW 79 AV@NW 50 ST. 1063 meters in 3 seconds. (1275 km/h)

High speed travel detected in trip 2106283: NW 36 ST@NW 79 AV to NW 79 AV@NW 50 ST. 1063 meters in 3 seconds. (1275 km/h)

High speed travel detected in trip 2101306: SW 211 ST@OP S DADE GOVT CTR to SW 216 ST@SW 103 AV. 1029 meters in 2 seconds. (1853 km/h)

High speed travel detected in trip 2101338: SW 211 ST@OP S DADE GOVT CTR to SW 216 ST@SW 103 AV. 1029 meters in 2 seconds. (1853 km/h)

Once you fix any error in Trapeze, you must regenerate the Google feed and revalidate to make sure you have corrected the error. 